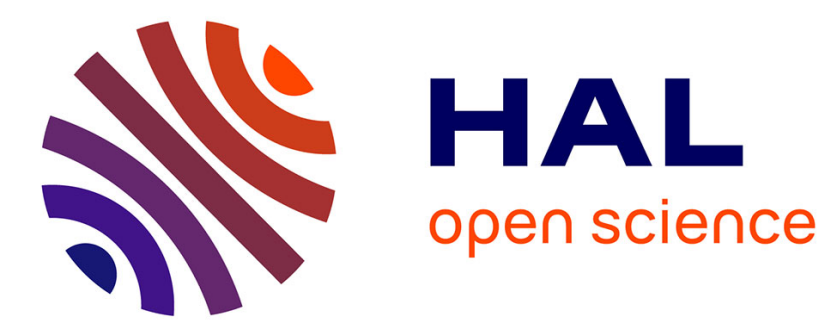

\title{
Inference for Archimax copulas
}

Simon Chatelain, Anne-Laure Fougères, Johanna G Nešlehová

\section{- To cite this version:}

Simon Chatelain, Anne-Laure Fougères, Johanna G Nešlehová. Inference for Archimax copulas. Annals of Statistics, In press, 10.1214/19-AOS1836 . hal-01816050

\section{HAL Id: hal-01816050 \\ https://hal.science/hal-01816050}

Submitted on 14 Jun 2018

HAL is a multi-disciplinary open access archive for the deposit and dissemination of scientific research documents, whether they are published or not. The documents may come from teaching and research institutions in France or abroad, or from public or private research centers.
L'archive ouverte pluridisciplinaire HAL, est destinée au dépôt et à la diffusion de documents scientifiques de niveau recherche, publiés ou non, émanant des établissements d'enseignement et de recherche français ou étrangers, des laboratoires publics ou privés. 


\title{
Inference for Archimax copulas
}

\author{
S. Chatelain*^, A.-L. Fougères*, and J. G. Nešlehová ${ }^{\dagger}$
}

May 30, 2018

\begin{abstract}
Archimax copula models can account for any type of asymptotic dependence between extremes and at the same time capture joint risks at medium levels. An Archimax copula $C_{\psi, \ell}$ is characterized by two functional parameters, the stable tail dependence function $\ell$, and the Archimedean generator $\psi$ which acts as a distortion of the extreme-value dependence model. This article develops semiparametric inference for Archimax copulas: a nonparametric estimator of $\ell$ and a moment-based estimator of $\psi$ assuming the latter belongs to a parametric family. Conditions under which $\psi$ and $\ell$ are identifiable are derived. The asymptotic behavior of the estimators is then established under broad regularity conditions; performance in small samples is assessed through a comprehensive simulation study. The Archimax copula model with the Clayton generator is then used to analyze monthly rainfall maxima at three stations in French Brittany. The model is seen to fit the data very well, both in the lower and in the upper tail. The nonparametric estimator of $\ell$ reveals asymmetric extremal dependence between the stations, which reflects heavy precipitation patterns in the area. Technical proofs, simulation results and $\mathrm{R}$ code are provided in the Appendix.
\end{abstract}

MSC 2010 subject classifications: Primary 62H12, 62G05, 62G20, 62G32; secondary 60G70.

Keywords: copulas, multivariate extremes, subasymptotic modeling, empirical processes.

\section{Introduction}

In various applications in environmental sciences, finance, insurance or risk management, joint extremal behavior between random variables is of particular interest. For example, this plays a central role in assessing risks of natural

*Univ Lyon, Université Claude Bernard Lyon 1, CNRS UMR 5208, Institut Camille Jordan, 43 blvd. du 11 novembre 1918, F-69622 Villeurbanne cedex, France

$\dagger$ Department of Mathematics and Statistics, McGill University, 805, rue Sherbrooke Ouest, Montréal (Québec) Canada H3A 0B9 
disasters and in determining the dimensions of structures such as dams or dikes. Misspecification of the dependence between the variables can lead to substantial underestimation of risk. To fix ideas, consider monthly maxima of daily precipitation for the months from September to February between 1976 and 2016 at three stations in French Brittany, Belle-Île-en-Mer, Groix, and Lorient. Based on these trivariate observations, provided by Météo France, the goal might be to assess the risk of medium and high precipitation at these three stations simultaneously in order to devise protective measures against floods in the region.

To answer questions such as the above, the so-called copula approach to multivariate data modeling has gained substantial popularity in recent years [30]. It is rooted in the decomposition of [45] which states that the joint distribution function of any multivariate random vector $\boldsymbol{X}=\left(X_{1}, \ldots, X_{d}\right)$ with continuous margins $F_{1}, \ldots, F_{d}$ can be written, for any $\boldsymbol{x} \in \mathbb{R}^{d}$, as

$$
\operatorname{Pr}\left(X_{1} \leq x_{1}, \ldots, X_{d} \leq x_{d}\right)=C\left\{F_{1}\left(x_{1}\right), \ldots, F_{d}\left(x_{d}\right)\right\}
$$

in terms of a unique copula $C$, that is, a distribution function on $[0,1]^{d}$ with standard uniform margins. This decomposition allows for separate modelling of the marginals $F_{1}, \ldots, F_{d}$ and the dependence structure $C$.

In the context of extremes, $C$ in (1.1) is typically chosen to be extreme-value. Following [27], this means that $C=C_{\ell}$ for some stable tail dependence function (stdf) $\ell$, that is, for any $\boldsymbol{u}=\left(u_{1}, \ldots, u_{d}\right) \in[0,1]^{d}$,

$$
C(\boldsymbol{u})=C_{\ell}(\boldsymbol{u})=\exp \left[-\ell\left\{-\log \left(u_{1}\right), \ldots,-\log \left(u_{d}\right)\right\}\right] .
$$

The use of extreme-value copulas is motivated by the fact that the latter are the only possible limits of normalized component-wise maxima on the uniform scale. However, while this asymptotic result is a very strong theoretical argument for using these models, it is seldom a realistic assumption in finite samples. For example, for the above precipitation data, the hypothesis that the underlying copula is extreme-value is clearly rejected by the test of [32] (p-val. $\approx 5 \times$ $\left.10^{-5}\right)$. While extreme-value dependence seems reasonable for seasonal maxima $(\mathrm{p}$-val. $\approx 0.43)$, working with the latter greatly reduces the sample size from $n=240$ to $n=40$. To estimate extremal dependence from monthly maxima directly, one could resort to the procedures in $[13,16]$. However, the latter approaches cannot assess joint risk in medium regimes, which can also be a cause for damaging events. Thus unified, smooth, and flexible yet parsimonious models that can capture risk at both medium and extreme regimes are needed, and only few such are available. Only recently, [39] proposed such a model for univariate non-zero precipitation amounts.

In the multivariate case when asymptotic dependence is present, the only dependence model available so far that is fully flexible in the extreme regime and can account for medium risks at the same time are the so-called Archimax copulas, proposed by [9] in the bivariate case and extended to higher dimensions in $[11,37]$. The latter are of the form

$$
C_{\psi, \ell}(\boldsymbol{u})=\psi\left[\ell\left\{\psi^{-1}\left(u_{1}\right), \ldots, \psi^{-1}\left(u_{d}\right)\right\}\right]
$$


at any $\boldsymbol{u} \in[0,1]^{d}$, where $\ell$ is an arbitrary $d$-variate stdf and $\psi:[0, \infty) \rightarrow[0,1]$ an Archimedean generator, specified in detail in Section 2. At this stage, $\psi$ may be thought of as a distortion function that contorts the extreme-value dependence model. Indeed, if $\psi(x)=\exp (-x), C_{\psi, \ell}$ becomes the extreme-value copula $C_{\ell}$. Moreover, as we recall in Section 2, $C_{\psi, \ell}$ is in the domain of attraction of $C_{\ell}$ under suitable conditions on $\psi[9,11]$.

Given that no inference is available, Archimax copulas have been rarely used in practice. The only viable option at present is to use a fully parametric Archimax model, where $\psi$ and $\ell$ belong to parametric classes of Archimedean generators and stdfs, respectively. In the bivariate and trivariate case, this has been employed in $[2,3]$. However, especially in higher dimensions, parametric models for $\ell$ are typically either too restrictive or too cumbersome.

This paper is the first to consider the statistical problem of fitting Archimax copulas to data in full generality. This amounts to estimating both $\ell$ and $\psi$. To accomplish this, we propose a semiparametric approach, in which $\ell$ is estimated nonparametrically and $\psi$ is assumed to belong to a parametric family $\Psi=$ $\left\{\psi_{\theta}, \theta \in \Theta\right\}$. Proceeding this way ensures the identifiability of $\ell$ and $\theta$ under mild conditions on $\Psi$. In addition, given that $\psi$ is a distortion function of the limiting extreme-value dependence, a parametric model for it is likely to be sufficiently rich to adequately capture dependence at medium and extreme levels. The estimators of $\ell$ developed here extend the ideas of $[8,41]$ and converge weakly to a centered Gaussian process under regularity conditions on $\ell$ and $\psi$. As we show, the Archimax copula model where $\Psi$ is the Clayton family fits the above mentioned monthly precipitation maxima very well, and reveals some particular aspects of the data that are climatologically sound. We also demonstrate that when the Clayton-Archimax model is appropriate, the nonparametric estimators of $\ell$ proposed here are considerably more precise and efficient than those in $[13,16]$, as well as the estimators based on seasonal blocks in [23].

The paper is organized as follows. Basic facts about Archimax copulas are recalled in Section 2, where the identifiability of $\psi$ and $\ell$ is proved under mild conditions on the family $\Psi$. Section 3 introduces nonparametric estimators of $\ell$ under the assumption that $\psi$ is known. Under the latter assumption and regularity conditions, the asymptotic behavior of these estimators is derived in Section 4, and their finite-sample performance is investigated via simulations in Section 5. The estimation of the Archimedean generator $\psi$ is then treated in Section 6 using a moment-based procedure. Section 7 is presents an application to precipitation data. Detailed proofs are reported in the Appendix; the latter also contains additional simulations.

In what follows, vectors in $\mathbb{R}^{d}$ are denoted by boldface letters, viz. $\boldsymbol{x}=$ $\left(x_{1}, \ldots, x_{d}\right)$. Binary operations such as $\boldsymbol{x}+\boldsymbol{y}$ or $\boldsymbol{a} \cdot \boldsymbol{x}, \boldsymbol{x}^{a}$ are understood as component-wise operations. In particular, for any function $f: \mathbb{R} \rightarrow \mathbb{R}$ and $\boldsymbol{x} \in \mathbb{R}^{d}, f(\boldsymbol{x})$ denotes the vector $\left(f\left(x_{1}\right), \ldots, f\left(x_{d}\right)\right)$. Furthermore, $\|\cdot\|$ stands for the $\ell_{1}$-norm, viz. $\|\boldsymbol{x}\|=x_{1}+\cdots+x_{d}$. For any $x, y \in \mathbb{R}$, let $x \wedge y=\min \{x, y\}$ and $x \vee y=\max \{x, y\}$. Finally, $\mathbb{R}_{+}^{d}$ is the positive orthant $[0, \infty)^{d}$ and for any $x \in \mathbb{R}, x_{+}$denotes the positive part of $x$. 


\section{Multivariate Archimax copulas}

This section gathers properties of Achimax copulas that are needed for subsequent developments. In Section 2.1, the existence, stochastic representation, and extremal behaivor is recalled, while Section 2.2 discusses the identifiability of $\psi$ and $\ell$.

\subsection{Existence and stochastic representation of Archimax copulas}

The class of Archimax copulas generalizes both the Archimedean and extremevalue copula families. On the one hand, when $\ell$ is the stdf pertaining to independence, i.e., $\ell(\boldsymbol{x})=x_{1}+\cdots+x_{d}$ for all $\boldsymbol{x} \in \mathbb{R}_{+}^{d}, C_{\psi, \ell}$ becomes the Archimedean copula $C_{\psi}$ with generator $\psi$ given, for all $\boldsymbol{u} \in[0,1]^{d}$ by

$$
C_{\psi}\left(u_{1}, \ldots, u_{d}\right)=\psi\left\{\phi\left(u_{1}\right)+\ldots+\phi\left(u_{d}\right)\right\},
$$

where $\phi:[0,1] \rightarrow[0, \infty)$ denotes the pseudo-inverse of $\psi$, given by $\phi(u)=$ $\psi^{-1}(u)$ for $u \in(0,1]$ and $\phi(0)=x_{\psi} \equiv \inf \{x \geq 0: \psi(x)=0\}$.

On the other hand, when $\psi(x)=\exp (-x)$ for any $x \geq 0, C_{\psi, \ell}$ becomes the extreme-value copula $C_{\ell}$ with stdf $\ell$.

For any generator $\psi$ of a $d$-variate Archimedean copula and any $d$-variate stdf $\ell, C_{\psi, \ell}$ given by (1.2) defines a copula, as proved by [11]. Recall from $[35,36,38]$ that $\psi$ is a generator of a $d$-variate Archimedean copula iff $\psi(0)=1$, $\psi(x) \rightarrow 0$ as $x \rightarrow \infty$ and if $\psi$ is $d$-monotone on $(0, \infty)$, i.e., $\psi$ has $d-2$ derivatives satisfying $(-1)^{k} \psi^{(k)} \geq 0$ on $(0, \infty)$ for $1 \leq k \leq d-2$ and that $(-1)^{d-2} \psi^{(d-2)}$ is non-increasing and convex on $(0, \infty)$. As proved in [43], $\ell: \mathbb{R}_{+}^{d} \rightarrow \mathbb{R}^{+}$is a $d$-variate stdf iff

(a) $\ell$ is homogeneous of degree 1 , i.e., for all $k>0$ and $x_{1}, \ldots, x_{d} \in[0, \infty)$, $\ell\left(k x_{1}, \ldots, k x_{d}\right)=k \ell\left(x_{1}, \ldots, x_{d}\right)$

(b) $\ell\left(\mathbf{e}_{1}\right)=\cdots=\ell\left(\mathbf{e}_{d}\right)=1$ where for $j=1, \ldots, d, \boldsymbol{e}_{j}$ denotes a $d$-dimensional vector whose components are all 0 except the $j$ th which is equal to 1 ;

(c) $\ell$ is fully $d$-max decreasing, i.e., for any $k \in \mathbb{N}, x_{1}, \ldots, x_{d}, h_{1}, \ldots, h_{d} \in$ $[0, \infty)$ and $J \subseteq\{1, \ldots, d\}$ with $|J|=k$,

$$
\sum_{\iota_{1}, \ldots, \iota_{k} \in\{0,1\}}(-1)^{\iota_{1}+\cdots+\iota_{k}} \ell\left(x_{1}+\iota_{1} h_{1} \mathbf{1}_{1 \in J}, \ldots, x_{d}+\iota_{d} h_{d} \mathbf{1}_{d \in J}\right) \leq 0 .
$$

Due to the homogeneity property (a), any stdf $\ell$ can be expressed, at any $\boldsymbol{x}=\mathbb{R}_{+}^{d}$ as $\ell(\boldsymbol{x})=\|\boldsymbol{x}\| A(\boldsymbol{x} /\|\boldsymbol{x}\|)$ where $A$ is the so-called Pickands dependence function [41] defined on the unit simplex

$$
\Delta_{d}=\left\{\boldsymbol{w} \in[0,1]^{d}: w_{1}+\ldots+w_{d}=1\right\} .
$$


Thus an Archimax copula $C_{\psi, \ell}$ can also be expressed, for any $\boldsymbol{u} \in[0,1]^{d}$, as

$$
C_{\psi, \ell}(\boldsymbol{u})=C_{\psi, A}(\boldsymbol{u})=\psi\left[\|\phi(\boldsymbol{u})\| A\left\{\frac{\phi(\boldsymbol{u})}{\|\phi(\boldsymbol{u})\|}\right\}\right] .
$$

Archimax copulas also admit a stochastic representation. Per Theorem 3.3 of [11], $C_{\psi, \ell}$ is the survival copula of a random vector

$$
\left(X_{1}, \ldots, X_{d}\right)=R \times\left(S_{1}, \ldots, S_{d}\right),
$$

where $R$ is a positive random variable independent of $\boldsymbol{S}$. The distribution function of $R$ is the inverse Williamson $d$-transform of $\psi$ and the survival function of $\boldsymbol{S}$ is given, for any $\boldsymbol{s} \in \mathbb{R}_{+}^{d}$, by $\operatorname{Pr}\left(S_{1}>s_{1}, \ldots, S_{d}>s_{d}\right)=[\max \{0,1-\ell(\boldsymbol{s})\}]^{d-1}$. In this representation, $R$ can again be interpreted as a distortion variable; when its law is Erlang with parameter $d, C_{\psi, \ell}=C_{\ell}$.

Finally, as announced in the introduction, Archimax copulas have a given extreme-value attractor. Recall that a function $f: \mathbb{R}_{+} \rightarrow \mathbb{R}_{+}$is regularly varying with index $\alpha \in \mathbb{R}$ iff for all $x>0, f(x t) / f(t) \rightarrow x^{\alpha}$ as $t \rightarrow \infty$, in notation $f \in \mathcal{R}_{\alpha}$. When $1-\psi(1 / \cdot) \in \mathcal{R}_{-\alpha}$ for $\alpha \in(0,1]$, it is shown in Proposition 6.1 in [11] that $C_{\psi, \ell}$ is in the maximum domain of attraction of the extreme-value copula $C_{\ell_{\alpha}}$, that is, for any $\boldsymbol{u} \in[0,1]^{d}, \lim _{n \rightarrow \infty} C_{\psi, \ell}^{n}\left(\boldsymbol{u}^{1 / n}\right)=C_{\ell_{\alpha}}(\boldsymbol{u})$, where for any $\boldsymbol{x} \in \mathbb{R}_{+}^{d}, \ell_{\alpha}(\boldsymbol{x})=\ell^{\alpha}\left(\boldsymbol{x}^{1 / \alpha}\right)$.

\subsection{Identifiability concerns}

In this section, we establish conditions under which $\ell$ and $\theta$ are identifiable when $\psi \in \Psi=\left\{\psi_{\theta}, \theta \in \Theta\right\}$. To accomplish this, we first consider two arbitrary $d$-variate Archimax copulas $C_{1}=C_{\psi_{1}, \ell_{1}}$ and $C_{2}=C_{\psi_{2}, \ell_{2}}$ whose generators $\psi_{k}, k \in\{1,2\}$ are not necessarily from a parametric class. The lemmas below investigate the question whether $C_{1}=C_{2}$ implies that the generators and stdfs are equal. All proofs are reported in Section A of the Appendix.

Lemma 2.1. Suppose that $C_{1}=C_{2}$ and $\psi_{1}=\psi_{2}=\psi$. Then $\ell_{1}=\ell_{2}$.

Lemma 2.2. Suppose that $C_{1}=C_{2}$ and $\ell_{1}=\ell_{2}=\ell$ is a $d$-variate stdf such that $\ell \neq \ell_{M}$, where for each $\boldsymbol{x} \in \mathbb{R}_{+}^{d}, \ell_{M}(\boldsymbol{x})=\max \left(x_{1}, \ldots, x_{d}\right)$. Suppose also that $\psi_{1}$ and $\psi_{2}$ are 2-monotone Archimedean generators. Then there exists a constant $c>0$ such that, for all $x \geq 0, \psi_{1}(x)=\psi_{2}(c x)$.

Next, note the following lemma. Its first part is an extension of Theorem 4.5.1 in [40] and has been shown in [25] in the case when $\psi$ is completely monotone. In the following, for any $\beta \in(0,1], \psi_{\beta}$ is defined by $\psi_{\beta}(t)=\psi\left(t^{\beta}\right)$ for all $t \geq 0$, and $\ell_{\beta}$ denotes $\ell^{\beta}\left(x_{1}^{1 / \beta}, \ldots, x_{d}^{1 / \beta}\right)$ for all $\boldsymbol{x} \in \mathbb{R}_{+}^{d}$.

Lemma 2.3. (i) Let $\psi$ be a d-monotone Archimedean generator, and let $0<\beta \leq 1$. Then $\psi_{\beta}$ is a d-monotone Archimedean generator.

(ii) Let $\ell$ be a d-variate stdf and $0<\beta \leq 1$. Then $\ell_{\beta}$ is a d-variate stdf. 
Now suppose that $\psi$ is a $d$-monotone Archimedean generator and $\ell$ an arbitrary $d$-variate stdf. By Lemma 2.3, $\psi_{\beta}$ is a $d$-monotone Archimedean generator and $\ell_{\beta}$ is a $d$-variate stdf for some $\beta \in(0,1]$. It is then easily seen that the Archimax copulas $C_{\psi_{\beta}, \ell}$ and $C_{\psi, \ell_{\beta}}$ are identical. One thus cannot expect $\ell$ to be unique and $\psi$ to be unique up to scaling. However, the following lemma shows that under a mild regularity condition on $\psi$, power transformations of $\psi$ and $\ell$ are the only possible sources of non-identifiability.

Lemma 2.4. Suppose that $\ell_{1} \neq \ell_{M}$ and $\ell_{2} \neq \ell_{M}$ are arbitrary $d$-variate stdfs and $\psi_{1}, \psi_{2}$ are $d$-monotone Archimedean generators with the property that for $k \in\{1,2\}, 1-\psi_{k}(1 / \cdot) \in \mathcal{R}_{-1 / m_{k}}$, with $m_{k} \geq 1$. Assuming, without loss of generality, that $m_{1} \leq m_{2}, C_{\psi_{1}, \ell_{1}}=C_{\psi_{2}, \ell_{2}}$ holds iff for all $\boldsymbol{x} \in \mathbb{R}_{+}^{d}$,

$$
\ell_{1}\left(x_{1}, \ldots, x_{d}\right)=\ell_{2}^{m_{1} / m_{2}}\left(x_{1}^{m_{2} / m_{1}}, \ldots, x_{d}^{m_{2} / m_{1}}\right)
$$

and there exists $c>0$ such that, for all $t \geq 0, \psi_{1}\left(c t^{m_{1} / m_{2}}\right)=\psi_{2}(t)$.

Lemma 2.4 allows us to formulate the following main result of this section that delineates the conditions under which an Archimax copula model is identifiable assuming that the Archimedean generator belongs to a parametric family. Its proof is a direct consequence of Lemma 2.4.

Proposition 2.1. Consider the class of d-variate Archimax copulas $\mathcal{C}_{\Psi}$ whose stdfs are arbitrary with $\ell \neq \ell_{M}$ and whose Archimedean generators belong to a parametric family $\Psi=\left\{\psi_{\theta}, \theta \in \Theta\right\}$. Assume also that the following conditions hold:

(i) For all $\theta \in \Theta, 1-\psi_{\theta}(1 / \cdot) \in \mathcal{R}_{-1 / m_{\theta}}$, with $m_{\theta} \geq 1$;

(ii) For all $\theta \in \Theta, c>0$ and $\beta>0, \psi_{\theta}\left(c t^{\beta}\right) \in \Psi$ implies that $c=1$ and $\beta=1$.

Then for arbitrary $C_{\psi_{\theta}, \ell}, C_{\psi_{\theta^{*}, \ell^{*}}} \in \mathcal{C}_{\Psi}, C_{\psi_{\theta}, \ell}=C_{\psi_{\theta^{*}, \ell^{*}}}$ holds if and only if $\ell=\ell^{*}$ and $\theta=\theta^{*}$.

Assumption (i) in Proposition 2.1 is Condition 4.1, which is anyway required for the nonparametric estimation of $\ell$ and discussed in detail in Section 4. As shown in [10], (i) holds for all Archimedean families in Tables 1 and 2 therein, that include Table 4.1 in [40]. Assumption (ii) is satisfied by most commonly used one-parameter families of Archimedean generators including the Frank, Clayton, and Ali-Mikhail-Haq models. The only exceptions we could find are Families 4.2.2, 4.2.4 (Gumbel), 4.2.12, and 4.2.18 in [40], and the outer power family $\phi_{1, \beta}$ from Theorem 4.5.1 therein. The nonidentifiability is not a concern for these models however, because the parameter $\theta$ is then absorbed by the stable tail dependence function using Lemma 2.4 so that the generator $\psi$ of the resulting Archimax model is a fixed function. 


\section{Estimation of the stdf}

In this section, we introduce two nonparametric estimators of the stdf $\ell$ of an Archimax copula $C_{\psi, \ell}$ under the assumption that the Archimedean generator $\psi$ is known. Recall that under this assumption, $\ell$ is unique, as explained in Section 2. Recall also that $\ell$ can be uniquely reconstructed from the corresponding Pickands dependence function $A$, and hence it suffices to estimate the latter. To see how to proceed, consider a random vector $\boldsymbol{U}$ distributed as an Archimax copula $C_{\psi, A}$ defined in (2.2). Introduce, for any $\boldsymbol{w}$ in the unit simplex $\Delta_{d}$ defined in (2.1),

$$
\xi(\boldsymbol{w})=\min _{1 \leq j \leq d}\left\{\frac{\phi\left(U_{j}\right)}{w_{j}}\right\}
$$

with the convention when $w_{j}=0$ for some $j=1, \ldots, d$. Then

$$
\operatorname{Pr}\{\xi(\boldsymbol{w})>x\}=C_{\psi, A}\{\psi(x \boldsymbol{w})\}=\psi\{x A(\boldsymbol{w})\} .
$$

Note in passing that when $\psi(x)=\exp (-x)$, that is, when $C_{\psi, A}$ is extremevalue, $\xi(\boldsymbol{w})$ is exponential with rate $A(\boldsymbol{w})$. This leads to the Pickands and the Capéraà-Fougères-Genest (CFG) type estimators of $A[8,19,22,41,49]$.

Now let $Z$ denote a random variable with survival function $\psi$, i.e., for all $x \geq 0, \operatorname{Pr}(Z>x)=\psi(x)$. Then for any $\boldsymbol{w} \in \Delta_{d}, \xi(\boldsymbol{w})$ has the same distribution as $Z / A(\boldsymbol{w})$. One gets in particular that

$$
\mathrm{E}\{\xi(\boldsymbol{w})\}=\frac{\mathrm{E}(Z)}{A(\boldsymbol{w})}, \quad \mathrm{E}[\log \{\xi(\boldsymbol{w})\}]=\mathrm{E}(\log Z)+\log \{A(\boldsymbol{w})\} .
$$

When $\psi$ is known, so are $\mathrm{E}(Z)$ and $\mathrm{E}(\log Z)$. Provided the latter are finite, (3.1) leads to the Pickands and CFG-type estimators of $A$, as explained next.

Let $\boldsymbol{X}_{1}, \boldsymbol{X}_{2}, \ldots, \boldsymbol{X}_{n}$ be a random sample from a $d$-variate distribution $H$ with unknown continuous margins $F_{1}, \ldots, F_{d}$ and an Archimax copula $C_{\psi, A}$ with known $\psi$ and unknown $A$. Because the margins are unknown, a sample of $C_{\psi, A}$ is not available, but as in $[19,23]$ one can base inference on the pseudoobservations given, for all $i \in\{1, \ldots, n\}$ and $j \in\{1, \ldots, d\}$ by

$$
\hat{U}_{i j}=\frac{n F_{n j}\left(X_{i j}\right)}{n+1},
$$

where $F_{n j}$ is the empirical distribution function of $X_{1 j}, \ldots, X_{n j}$, for any $j \in$ $\{1, \ldots, d\}$. Now define for every $\boldsymbol{w} \in \Delta_{d}$, and $i \in\{1, \ldots, n\}$,

$$
\hat{\xi}_{i}(\boldsymbol{w})=\min _{1 \leq j \leq d} \frac{\phi\left(\hat{U}_{i j}\right)}{w_{j}},
$$

again with the convention that $\phi\left(\hat{U}_{i j}\right) / w_{j}=\infty$ when $w_{j}=0$. However, note that for any $\boldsymbol{w} \in \Delta_{d}, w_{j}>0$ for at least one $j$, so that $\hat{\xi}_{i}(\boldsymbol{w})$ is finite for every $i=1, \ldots, n$. Then, provided that $\mathrm{E}(Z)$ exists, the Pickands-type estimator $A_{n}^{\mathrm{P}}$ is defined, for any $\boldsymbol{w} \in \Delta_{d}$, by

$$
A_{n}^{\mathrm{P}}(\boldsymbol{w})=\frac{n \mathrm{E}(Z)}{\sum_{i=1}^{n} \hat{\xi}_{i}(\boldsymbol{w})} .
$$


Similarly, if $\mathrm{E}(\log Z)$ exists, the CFG-type estimator $A_{n}^{\mathrm{CFG}}$ is defined through

$$
\log A_{n}^{\mathrm{CFG}}(\boldsymbol{w})=\mathrm{E} \log Z-\frac{1}{n} \sum_{i=1}^{n} \log \hat{\xi}_{i}(\boldsymbol{w}) .
$$

When $\psi(x)=\exp (-x)$, one has that $\mathrm{E}(Z)=1$ and $\mathrm{E}(\log Z)=-\gamma$, where $\gamma$ is the Euler-Mascheroni constant, and $A_{n}^{\mathrm{P}}$ and $A_{n}^{\mathrm{CFG}}$ reduce to the rank based Pickands and CFG estimators investigated in [19, 23].

Note that in general, neither $A_{n}^{\mathrm{P}}$ or $A_{n}^{\mathrm{CFG}}$ is a genuine Pickands dependence function. In order to enforce the endpoint constraints $A\left(\boldsymbol{e}_{j}\right)=1$ for $j=1, \ldots, d$, introduce

$$
\hat{\mu}=\frac{1}{n} \sum_{i=1}^{n} \phi\left(\frac{i}{n+1}\right), \quad \hat{\nu}=\frac{1}{n} \sum_{i=1}^{n} \log \phi\left(\frac{i}{n+1}\right) .
$$

The endpoint-corrected Pickands and CFG-type estimators now arise by replacing $\mathrm{E}(Z)$ by $\hat{\mu}$ in (3.3) and $\mathrm{E}(\log Z)$ by $\hat{\nu}$ in (3.4), respectively, viz.

$$
A_{n, c}^{\mathrm{P}}(\boldsymbol{w})=\frac{n \hat{\mu}}{\sum_{i=1}^{n} \hat{\xi}_{i}(\boldsymbol{w})}, \quad \log A_{n, c}^{\mathrm{CFG}}(\boldsymbol{w})=\hat{\nu}-\frac{1}{n} \sum_{i=1}^{n} \log \hat{\xi}_{i}(\boldsymbol{w}) .
$$

These corrected versions avoid the generally cumbersome computation of $\mathrm{E}(Z)$ or $\mathrm{E}(\log Z)$. In addition, the following holds, owing to the fact that $\hat{\mu}=$ $1 / n \sum_{i=1}^{n} \phi\left(\hat{U}_{i j}\right)$ and $\hat{\nu}=1 / n \sum_{i=1}^{n} \log \phi\left(\hat{U}_{i j}\right)$ a.s. for all $j \in\{1, \ldots, d\}$.

Proposition 3.1. For $j=1, \ldots$, d, it holds that $A_{n, c}^{P}\left(\boldsymbol{e}_{j}\right)=1$ and $A_{n, c}^{C F G}\left(\boldsymbol{e}_{j}\right)=$ 1 a.s. Moreover, one also has a.s. that $A_{n, c}^{P}(\boldsymbol{w}) \geq \max _{1 \leq j \leq d} w_{j}$ and $A_{n, c}^{C F G}(\boldsymbol{w}) \geq$ $\max _{1 \leq j \leq d} w_{j}$ for all $\boldsymbol{w} \in \Delta_{d}$.

Note that when $d=2$ and $\psi(x)=\exp (-x), A_{n, c}^{\mathrm{P}}$ is the corrected rank-based Pickands estimator from [19] with end-point correction as in [24].

\section{Asymptotic behavior}

In this section, we investigate the asymptotic behavior of the Pickands and CFGtype estimators defined in the previous section, under the assumption that $\psi$ is known. We first detail the required conditions on $\psi$ and $\ell$ in Section 4.1, and study, in Section 4.2, the limiting behavior of the processes

$$
\mathbb{A}_{n}^{\mathrm{P}}=\sqrt{n}\left(A_{n}^{\mathrm{P}}-A\right) \quad \text { and } \quad \mathbb{A}_{n}^{\mathrm{CFG}}=\sqrt{n}\left(A_{n}^{\mathrm{CFG}}-A\right) .
$$

The main ingredients of the proof are then made explicit in Section 4.3.

\subsection{Conditions}

Conditions on $\psi$ are stated, followed by conditions on $\ell$. 
Condition 4.1. For $d \geq 2, \psi$ is a d-monotone Archimedean generator and $1-\psi(1 / x) \in \mathcal{R}_{-1 / m}$ for some $m \geq 1$.

Condition 4.1, which is equivalent to $\phi(1-1 / x) \in \mathcal{R}_{-m}$, is very general and satisfied by virtually all $d$-monotone Archimedean generators [10,33]. This is because it holds whenever $1 / R$ with $R$ as in (2.3) is in the domain of attraction of the Fréchet $\left(\Phi_{\alpha}\right)$, Gumbel $(\Lambda)$ or Weibull $\left(\Psi_{\alpha}\right)$ distributions for some $\alpha>0$, in notation $1 / R \in \mathcal{M}\left(\Phi_{\alpha}\right), 1 / R \in \mathcal{M}(\Lambda)$ or $1 / R \in \mathcal{M}\left(\Psi_{\alpha}\right)$. Moreover, Condition 4.1 with $m=1$ further holds as soon as $\mathrm{E}\left(1 / R^{1+\epsilon}\right)<\infty$ for some $\epsilon>0$, see Proposition 2 in [4].

Condition 4.2. For $d \geq 2, \psi$ is a d-monotone Archimedean generator that satisfies either

(a) $\psi \in \mathcal{R}_{-s}$ for $s>0$;

(b) $\psi \in \mathcal{M}(\Lambda)$;

(c) $\psi^{-1}(0)<\infty$ and $\psi\left\{\psi^{-1}(0)-1 / x\right\} \in \mathcal{R}_{-\alpha-d+1}$ for $\alpha>0$.

Most Archimedean generators indeed satisfy Condition 4.2. As shown in [33], Condition 4.2 (a) holds whenever $R$ in (2.3) is such that $R \in \mathcal{M}\left(\Phi_{s}\right)$ and is further equivalent to $\phi(1 / x) \in \mathcal{R}_{1 / s}$. Furthermore, Condition 4.2 (b) holds whenever $R \in \mathcal{M}(\Lambda)$ and this happens if and only if $\phi(1 / x)$ is $\Pi$-varying as defined, e.g., in [42, Section 0.4.3]. Finally, Condition 4.2 (c) is equivalent to $R \in \mathcal{M}\left(\Psi_{\alpha}\right)$ and further to $\left\{\psi^{-1}(0)-\phi(1 / x)\right\} \in \mathcal{R}_{-1 /(\alpha+d-1)}$.

Condition 4.3. For $d \geq 2$, $\ell$ is a d-variate stdf that is twice continuously differentiable and for which there exists $M>0$ such that for any $i \neq j \in$ $\{1, \ldots, d\}$ and any $\boldsymbol{x} \in(0, \infty)^{d}$,

$$
-\frac{\partial^{2}}{\partial x_{i} \partial x_{j}} \ell\left(x_{1}, \ldots, x_{d}\right) \equiv-\ddot{\ell}_{i j}\left(x_{1}, \ldots, x_{d}\right) \leq M\left(\frac{1}{x_{i}} \wedge \frac{1}{x_{j}}\right) .
$$

Condition 4.3 extends Condition (5.2) in [44] to the case of $d>2$. The following example demonstrates that it is satisfied by the logistic stdf.

Example 4.1. The logistic stdf is given for any $\boldsymbol{x} \in \mathbb{R}_{+}^{d}$ and $\theta \geq 1$ by $\ell_{\theta}\left(x_{1}, \ldots, x_{d}\right)=$ $\left(x_{1}^{\theta}+\ldots+x_{d}^{\theta}\right)^{\frac{1}{\theta}}$. It is easily seen that for any $\boldsymbol{x} \in \mathbb{R}_{+}^{d}$

$$
-\ddot{\ell}_{i j}(\boldsymbol{x})=(\theta-1) x_{i}^{\theta-1} x_{j}^{\theta-1}\left(x_{1}^{\theta}+\ldots+x_{d}^{\theta}\right)^{\frac{1}{\theta}-2} \leq(\theta-1)\left(\frac{1}{x_{i}} \wedge \frac{1}{x_{j}}\right) .
$$

The following Lemma, proved in Section 10.1 of the Appendix, explains that under Conditions 4.1 and 4.2, the Pickands and CFG-type estimators are indeed well-defined and have the same limiting behavior as their end-point corrected versions.

Lemma 4.1. (i) Suppose that $\psi$ is differentiable on $(0, \infty)$ and satisfies either Condition 4.2 (a) with $s>1$, (b) or (c). Then $\mathrm{E}(Z)<\infty$ and $\hat{\mu} \rightarrow \mathrm{E}(Z)$ as $n \rightarrow \infty$.

(ii) Suppose that $\psi$ is differentiable on $(0, \infty)$ and satisfies Conditions 4.1 and 4.2. Then $\mathrm{E}(\log Z)<\infty$ and $\hat{\nu} \rightarrow \mathrm{E}(\log Z)$ as $n \rightarrow \infty$. 


\subsection{Main results}

First note that the interior of the unit simplex satisfies

$$
\stackrel{\circ}{\Delta}_{d}=\left\{\boldsymbol{w} \in[0,1]^{d}: w_{1}+\ldots+w_{d}=1, w_{(1)}>0\right\},
$$

where $w_{(1)}=\min \left(w_{1}, \ldots, w_{d}\right)$. To simplify notation, write, for any $\boldsymbol{x} \in \mathbb{R}_{+}^{d}$, $\psi(\boldsymbol{x})=\left(\psi\left(x_{1}\right), \ldots, \psi\left(x_{d}\right)\right)$. Furthermore, for any compact subset $\mathcal{K}$ of $\stackrel{\triangleright}{d}_{d}$, let $\mathcal{C}(\mathcal{K})$ denote the space of continuous functions on $\mathcal{K}$ equipped with the supnorm. For a $d$-variate copula $C$, let $\alpha$ be a $C$-Brownian bridge, i.e., a tight, centered Gaussian process with covariance function given, for all $\boldsymbol{u}, \boldsymbol{v} \in[0,1]^{d}$ by $\operatorname{Cov}\{\alpha(\boldsymbol{u}), \alpha(\boldsymbol{v})\}=C(\boldsymbol{u} \wedge \boldsymbol{v})-C(\boldsymbol{u}) C(\boldsymbol{v})$. For any $j \in\{1, \ldots, d\}$ and $\boldsymbol{u} \in$ $[0,1]^{d}$, let also $\dot{C}_{j}(\boldsymbol{u})=\left(\partial / \partial u_{j}\right) C(\boldsymbol{u})$; if the latter derivative does not exist, set $\dot{C}_{j}(\boldsymbol{u})=\lim \sup _{h \rightarrow 0}\left\{C\left(\boldsymbol{u}+h \boldsymbol{e}_{j}\right)-C(\boldsymbol{u})\right\}$. Finally, let $\mathbb{C}$ be the process defined, for any $\boldsymbol{u} \in[0,1]^{d}$, by

$$
\mathbb{C}(\boldsymbol{u})=\alpha(\boldsymbol{u})-\sum_{j=1}^{d} \dot{C}_{j}(\boldsymbol{u}) \alpha\left(\boldsymbol{u}^{(j)}\right)
$$

with $\boldsymbol{u}^{(j)}=\left(1, \ldots, 1, u_{j}, 1, \ldots, 1\right)$. Theorems 4.1 and 4.2 below respectively specify the limiting behavior of the processes $\mathbb{A}_{n}^{C F G}$ and $\mathbb{A}_{n}^{\mathrm{P}}$ defined in (4.1). These convergence results require an alpha-mixing sequence of random variables $\boldsymbol{X}_{i}$ with a time-invariant Archimax copula. This allows to forgo independence for a form of asymptotic independence in time.

Definition 4.1. For $-\infty \leq a<b \leq \infty$, let $\mathcal{F}_{a}^{b}$ be the $\sigma$-field generated by the $\boldsymbol{X}_{i}$ with $i \in\{a, a+1, \ldots, b\}$. For $k \geq 1$, define

$$
\alpha^{[\boldsymbol{X}]}(k)=\sup \left\{|\operatorname{Pr}(A \cap B)-\operatorname{Pr}(A) \operatorname{Pr}(B)|: A \in \mathcal{F}_{-\infty}^{i}, B \in \mathcal{F}_{i+k}^{\infty}, i \in \mathbb{Z}\right\}
$$

as the alpha-mixing coefficient of $\left(\boldsymbol{X}_{i}\right)_{i \in \mathbb{Z}}$. The series is called alpha-mixing (or strongly mixing) if $\alpha^{[\boldsymbol{X}]}(k) \rightarrow 0$ as $k \rightarrow \infty$.

Theorem 4.1. Suppose that $\boldsymbol{X}_{1}, \boldsymbol{X}_{2}, \ldots$ is a stationary, alpha-mixing sequence with $\alpha^{[\boldsymbol{X}]}(k)=O\left(a^{k}\right)$, as $k \rightarrow \infty$, for some $a \in(0,1)$. Suppose that the marginals of the stationary distribution are continuous and the corresponding copula $C=C_{\psi, \ell}=C_{\psi, A}$ is Archimax with generator $\psi$ that is q-monotone for some $q \geq 3$ and such that $\psi^{\prime \prime}$ exists and is continuous on $(0, \infty)$. Further assume that Conditions 4.1 and 4.3 hold, and that either Condition 4.2 (a) is satisfied or Condition 4.2 (b) is satisfied with the additional requirement that $-\log (\psi)$

is concave on $\left(0, x_{\psi}\right)$. Then for any compact set $\mathcal{K} \subset \stackrel{\circ}{\Delta}_{d}, \mathbb{A}_{n}^{C F G} \rightsquigarrow \mathbb{A}^{C F G}$ as $n \rightarrow \infty$ in $\mathcal{C}(\mathcal{K})$, where for any $\boldsymbol{w} \in \stackrel{\triangleright}{\Delta}_{d}$,

$$
\mathbb{A}^{C F G}(\boldsymbol{w})=A(\boldsymbol{w}) \int_{0}^{1} \mathbb{C}[\psi\{-\boldsymbol{w} \log (u)\}] \frac{d u}{u \log u} .
$$


Theorem 4.2. Under the hypotheses of Theorem 4.1 and the additional assumption that $s>2$ in case Condition 4.2 (a) holds, one has that, for any compact set $\mathcal{K} \subset \stackrel{\circ}{d}_{d}, \mathbb{A}_{n}^{P} \rightsquigarrow \mathbb{A}^{P}$ as $n \rightarrow \infty$ in $\mathcal{C}(\mathcal{K})$, where for any $\boldsymbol{w} \in \stackrel{\circ}{d}_{d}$,

$$
\mathbb{A}^{P}(\boldsymbol{w})=\frac{-A^{2}(\boldsymbol{w})}{E(Z)} \int_{0}^{1} \mathbb{C}[\psi\{-\boldsymbol{w} \log (u)\}] \frac{d u}{u} .
$$

First observe that the conditions of Theorem 4.2 are stronger than those of Theorem 4.1; this is further investigated in Section 9.2 of the Appendix. Also note that the generator given, for all $x \geq 0$, by $\psi(x)=\exp (-x)$ is completely monotone and satisfies Conditions 4.1 and 4.2 (b) and is such that $-\log (\psi)$ is linear. Hence, Theorems 4.1 and 4.2 remain valid in the special case when $C$ is extreme-value. Finally, note that because of Lemma 4.1, the asymptotic behavior for the endpoint corrected versions of the CFG and Pickands-type estimators is the same, as stated below.

Corollary 4.1. Theorems 4.1 and 4.2 also hold when $\mathbb{A}_{n}^{C F G}$ and $\mathbb{A}_{n}^{P}$ are respectively replaced by the processes $\mathbb{A}_{n, c}^{C F G}=\sqrt{n}\left(A_{n, c}^{C F G}-A\right)$ and $\mathbb{A}_{n, c}^{P}=\sqrt{n}\left(A_{n, c}^{P}-A\right)$.

\subsection{Outline of the proofs of Theorems 4.1 and 4.2}

To establish weak convergence of $\mathbb{A}_{n}^{\mathrm{CFG}}$ and $\mathbb{A}_{n}^{\mathrm{P}}$, the weak convergence of the empirical copula process with respect to weighted metrics established in [6] is used.

The result of [6] requires smoothness assumptions that are recalled as Conditions 9.1 and 9.2 in Section 9 of the Appendix. We start by verifying that the latter indeed hold for Archimax copulas under suitable assumptions on the generator and the stdf, and this is nontrivial. Proposition 4.1 below follows from Propositions 1 and 2 that are stated and proved in Section 9.2 of the Appendix.

Proposition 4.1. Under the hypotheses of Theorem 4.1, Conditions 9.1 and 9.2 are satisfied.

Remark 4.1. Proposition 4.1 also shows that Condition (4.1) in [44] holds for an Archimedean copula $C_{\psi}$ if $\psi$ is q-monotone for some $q \geq 3, \psi^{\prime \prime}$ exists and is continuous on $(0, \infty)$, Condition 4.1 holds, and either Condition 4.2 (a) is satisfied or Condition 4.2 (b) is satisfied with the additional requirement that $-\log (\psi)$ is concave.

Following [19], we introduce the processes defined, for any $\boldsymbol{w} \in \Delta_{d}$, by

$$
\begin{aligned}
\mathbb{B}_{n}^{\mathrm{CFG}}(\boldsymbol{w}) & =\sqrt{n}\left\{\log A_{n}^{\mathrm{CFG}}(\boldsymbol{w})-\log A(\boldsymbol{w})\right\} \\
\mathbb{B}_{n}^{\mathrm{P}}(\boldsymbol{w}) & =\sqrt{n}\left\{1 / A_{n}^{\mathrm{P}}(\boldsymbol{w})-1 / A(\boldsymbol{w})\right\} .
\end{aligned}
$$

The following Lemma establishes that these processes are functionals of the empirical copula process defined by $\hat{\mathbb{C}}_{n}(\boldsymbol{u})=\sqrt{n}\left\{\hat{C}_{n}(\boldsymbol{u})-C(\boldsymbol{u})\right\}$ for any $\boldsymbol{u} \in$ $[0,1]^{d}$, where $\hat{C}_{n}(\boldsymbol{u})=1 / n \sum_{i=1}^{n} \prod_{j=1}^{d} \mathbb{1}\left(\hat{U}_{i j} \leq u_{j}\right)$ denotes the empirical copula, in terms of the pseudo-observations $\hat{U}_{i j}$ specified in (3.2). 
Lemma 4.2. Fix an arbitrary $\boldsymbol{w} \in \Delta_{d}$. Then, provided $E(\log Z)$ exists,

$$
\mathbb{B}_{n}^{C F G}(\boldsymbol{w})=-\int_{0}^{1} \hat{\mathbb{C}}_{n}[\psi\{-\boldsymbol{w} \log (u)\}] \frac{d u}{u \log u} .
$$

Furthermore, provided $E(Z)$ exists,

$$
\mathbb{B}_{n}^{P}(\boldsymbol{w})=\frac{1}{E(Z)} \int_{0}^{1} \hat{\mathbb{C}}_{n}[\psi\{-\boldsymbol{w} \log (u)\}] \frac{d u}{u} .
$$

The proof is relegated to Section 10.1 of the Appendix. Recall that the required existence of the expectations $E(\log Z)$ and $E(Z)$ is treated in Lemma 4.1 and is satisfied under the hypotheses of Theorems 4.1 and 4.2 , respectively. Weak convergence of $\mathbb{B}_{n}^{C F G}$ and $\mathbb{B}_{n}^{\mathrm{P}}$ is established next. The proof is provided in Sections 10.3 and 10.4 of the Appendix.

Proposition 4.2. Let $\mathcal{K}$ be any compact subset of $\AA_{d}$.

(a) Under the hypotheses of Theorem $4.1, \mathbb{B}_{n}^{C F G} \rightsquigarrow \mathbb{B}^{C F G}$ as $n \rightarrow \infty$ in $\mathcal{C}(\mathcal{K})$, where for any $\boldsymbol{w} \in \stackrel{\circ}{\Delta}_{d}$,

$$
\mathbb{B}^{C F G}(\boldsymbol{w})=\int_{0}^{1} \mathbb{C}[\psi\{-\boldsymbol{w} \log (u)\}] \frac{d u}{u \log u} .
$$

(b) Under the hypotheses of Theorem $4.2, \mathbb{B}_{n}^{P} \rightsquigarrow \mathbb{B}^{P}$ as $n \rightarrow \infty$ in $\mathcal{C}(\mathcal{K})$, where for any $\boldsymbol{w} \in \stackrel{\circ}{\Delta}_{d}$,

$$
\mathbb{B}^{P}(\boldsymbol{w})=\frac{1}{E(Z)} \int_{0}^{1} \mathbb{C}[\psi\{-\boldsymbol{w} \log (u)\}] \frac{d u}{u} .
$$

The validity of Theorem 4.1 now follows directly from Proposition 4.2 (a) and Theorem 3.9.4. of [46], since the map $\eta: \mathcal{C}(\mathcal{K}) \rightarrow \mathcal{C}(\mathcal{K})$ defined by $\eta(f)=\exp (f)$ is Hadamard differentiable. Similarly, Theorem 4.2 is a direct consequence of Proposition 4.2 (b) and Slutsky's Lemma, as for any $\boldsymbol{w} \in \Delta_{d}$,

$$
\mathbb{A}_{n}^{\mathrm{P}}(\boldsymbol{w})=\frac{-A^{2} \mathbb{B}_{n}^{\mathrm{P}}(\boldsymbol{w})}{1+n^{-1 / 2} A(\boldsymbol{w}) \mathbb{B}_{n}^{\mathrm{P}}(\boldsymbol{w})} .
$$

Remark 4.2. Theorems 4.1 and 4.2 can in fact be shown to hold for any compact $\mathcal{K}$ subset of $\dot{\Delta}_{d}=\left\{\boldsymbol{w} \in[0,1]^{d}: w_{1}+\ldots+w_{d}=1, w_{(d)}<1\right\}$, where $w_{(d)}=\max \left(w_{1}, \ldots, w_{d}\right)$. Such sets allow for several components $w_{j}$ of $\boldsymbol{w}$ to be equal to zero. In that case, Proposition 4.2 can be proved as follows. Let $\mathcal{K}$ be any compact subset of $\dot{\Delta}_{d}$. For any $\boldsymbol{w} \in \mathcal{K}$, let $\boldsymbol{w}^{\star}=\left\{w_{j}: w_{j}>0, j=1, \ldots, d\right\}$. Thus $\boldsymbol{w}^{\star}$ is a $d^{\star}$-dimensional vector, with $d^{\star} \leq d$, and

$$
\mathbb{B}_{n}^{C F G}(\boldsymbol{w})=-\int_{0}^{\infty} \hat{\mathbb{C}}_{n}^{\star}\left\{\psi\left(\boldsymbol{w}^{\star} x\right)\right\} \frac{d x}{x}, \quad \mathbb{B}_{n}^{P}(\boldsymbol{w})=\frac{1}{E(Z)} \int_{0}^{\infty} \hat{\mathbb{C}}_{n}^{\star}\left\{\psi\left(\boldsymbol{w}^{\star} x\right)\right\} d x,
$$


where $\hat{\mathbb{C}}_{n}^{\star}=\sqrt{n}\left(\hat{C}_{n}^{\star}-C^{\star}\right)$. Note that $C^{\star}=C_{\psi, \ell^{\star}}$ has the same Archimedean generator $\psi$ as $C$, and the marginal stdf $\ell^{\star}$ defined as the original $\ell$ with zero arguments corresponding to the zeros of $\boldsymbol{w}$. It is then possible to find a $K \in$ $\mathbb{N}$ such that $\mathcal{K} \subset B_{1 / K}=\left\{\boldsymbol{w} \in[0,1]^{d}: w_{1}+\ldots+w_{d}=1, w_{(1)}^{\star} \geq 1 / K\right\}$, where $w_{(1)}^{\star}=\min \left\{w_{j}: w_{j}>0\right\}$. The rest of the proof is identical to that of Proposition 4.2. Extending the weak convergence to the entire unit simplex $\Delta_{d}$ would require a different approach, and it remains to be seen whether such an extension is possible at all.

\section{Simulation Study}

We investigate the performance of the endpoint-corrected estimators defined in (3.5) through simulations using R package simsalapar [26]. The design is as follows: (i) dimension $d \in\{2,4,10\}$; (ii) sample size $n \in\{200,500,1000\}$; (iii) Archimedean generator from the Clayton, Gumbel, Frank and Joe families [40]; (iv) Pickands dependence functions from the following families: Logistic (LG), scaled negative extremal Dirichlet (NSD) of [4], and the discrete spectral measure (DSM) of [15].

The parameters of the Archimedean generator and the Pickands dependence function were chosen as to cover various scenarios in terms of association, lower/upper tail dependence, and asymmetry. We also intentionally challenge Conditions 4.1-4.3 to explore the robustness of the convergence results. For the sake of brevity, we present the main conclusions of this simulation study and provide representative illustrations; the complete results are available in Section 11 of the Appendix. To evaluate the performance of the estimators, the integrated squared error (ISE) and integrated relative absolute error (IRAE) defined below were used.

$$
\begin{aligned}
\operatorname{ISE}\left(A_{n}\right) & =\frac{1}{\left|\Delta_{d}\right|} \int_{\Delta_{d}}\left\{A_{n}(\boldsymbol{w})-A(\boldsymbol{w})\right\}^{2} d \boldsymbol{w}, \\
\operatorname{IRAE}\left(A_{n}\right) & =\frac{1}{\left|\Delta_{d}\right|} \int_{\Delta_{d}} \frac{\left|A_{n}(\boldsymbol{w})-A(\boldsymbol{w})\right|}{A(\boldsymbol{w})} d \boldsymbol{w} .
\end{aligned}
$$

ISE and IRAE were computed using Monte-Carlo integration with 10000 uniformly distributed samples on $\Delta_{d}$. For each scenario, 1000 Monte-Carlo replicates were deemed sufficient to capture the behavior of ISE and IRAE.

\subsection{Comparisons between the Pickands and the CFG-type estimators}

We first compared the Pickands and the CFG-type estimators in various scenarios; the results are reported in Tables D1-D6 in the Appendix. Figure 1 is representative of the overall pattern, namely that the CFG-type estimator performs better on average both in terms of ISE and IRAE. This is in accordance

with the findings of [19], who reported that the CFG estimator is generally more 

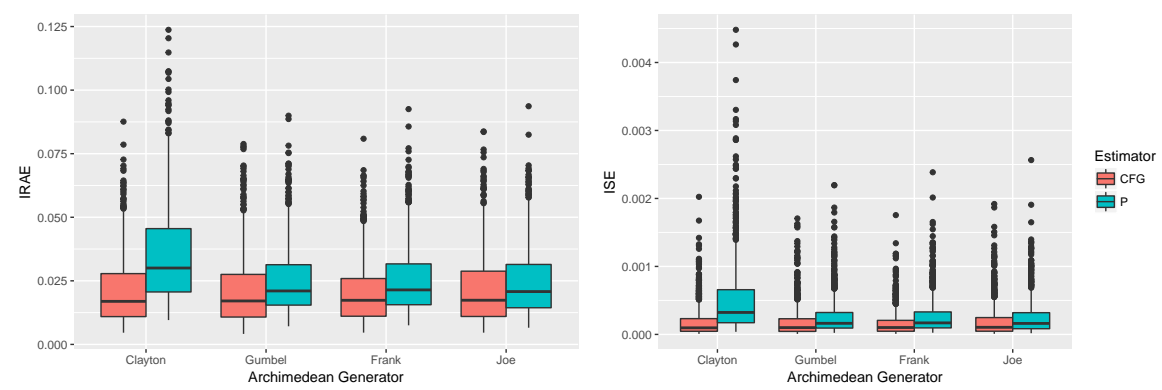

Figure 1: Boxplots of $\operatorname{IRAE}\left(A_{n, c}\right)$ (left) and $\operatorname{ISE}\left(A_{n, c}\right)$ (right) for the Pickands (green) and CFG (orange) type estimators for $n=200, d=4$, various Archimedean generators with $\tau(\psi)=1 / 5$ and the NSD Pickands dependence function with parameters $\boldsymbol{\alpha}=(1,2,3,4), \rho=0.59$.
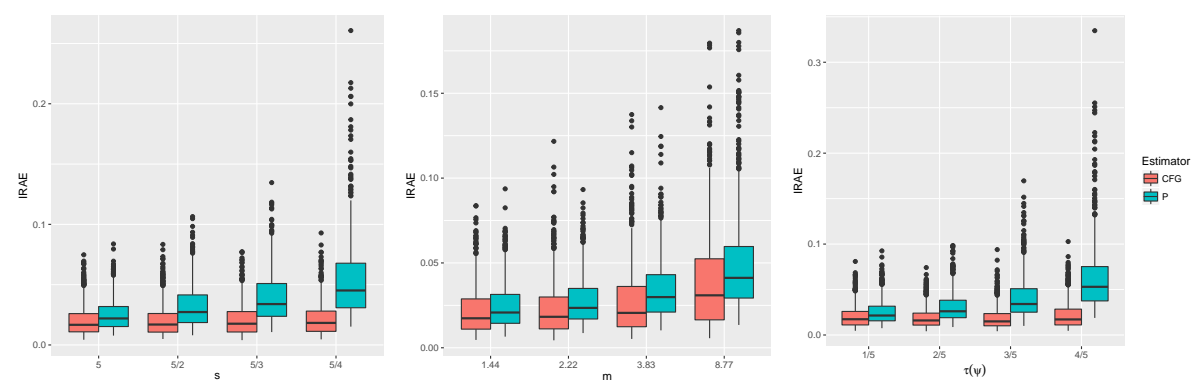

Figure 2: Boxplots of IRAE for the Pickands (green) and CFG (orange) estimators for $n=200, d=4$, and the Clayton generator $\psi$ with $\theta=1 / s$ for various values of $s$ (left), the Joe generator for various values of $\theta=m$ (middle) and Frank for various values of $\tau(\psi)=1-(4 / \theta)\left\{1-D_{1}(\theta)\right\}$ (right), where $D_{1}$ denotes the Debye function. The Pickands dependence function is NSD with parameters $\boldsymbol{\alpha}=(1,2,3,4), \rho=0.59$.

efficient than the Pickands estimator in the bivariate case. Also apparent from Figure 1 is that IRAE is more revealing than ISE, which is why we concentrate on the former henceforth.

Given that the behavior of $\psi$ at 0 and $\infty$ played a key role in the conditions of Theorems 4.1 and 4.2, we next investigate the impact of the index of regular variation of $\psi$ and $1-\psi(1 / \cdot)$. Figure 2 shows the performance of the estimators for the NSD Pickands dependence function with parameters $\boldsymbol{\alpha}=(1,2,3,4)$, $\rho=0.59$. In the left panel, the generator is Clayton with parameter $\theta$; the latter satisfies Condition 4.2 (a) with $s=1 / \theta$. This plot reveals that decreasing $s$ has a detrimental effect on $A_{n, c}^{\mathrm{P}}$ while $A_{n, c}^{\mathrm{CFG}}$ is hardly affected. When $s \leq 2$, conditions of Theorem 4.2 are no longer met; it is therefore not surprising that the behavior of $A_{n, c}^{\mathrm{P}}$ deteriorates quickly as $s \rightarrow 0$. The middle panel of Figure 2 explores the effect of $m$ when the generator is Joe, which satisfies Condition 
4.1 with $\theta=m$. One can again see that $A_{n, c}^{\mathrm{P}}$ performs worse than $A_{n, c}^{\mathrm{CFG}}$, but this time, increasing $m$ has a negative effect on both estimators. Finally, the right panel of Figure 2 shows the effect of dependence of the Archimedean copula $C_{\psi}$ with generator $\psi$ measured by $\tau(\psi)$, Kendall's tau of the bivariate Archimedean copula with generator $\psi$, for the Frank generator. In this case, $m=1$, and increasing $\tau(\psi)$ negatively affects both estimators, although $A_{n, c}^{\mathrm{CFG}}$ is less sensitive.

\subsection{The effect of the sample size, dimension, and depen- dence}

Given that the CFG-type estimator performed consistently better than $A_{n, c}^{\mathrm{P}}$, we concentrate on the former hereafter and explore the effect of sample size, dimension and dependence. We choose the Pickands dependence function $A$ to be either LG with parameter $\varrho=1 / 2$ (all dimensions) or NSD with parameters $\boldsymbol{\alpha}=(1,2), \rho=0.59$ (for $d=2$ ), $\boldsymbol{\alpha}=(1,2,3,4), \rho=0.59$ (for $d=4$ ) and $\boldsymbol{\alpha}=(1,1,1,1,2,2,2,3,3,4), \rho=0.69$ (for $d=10$ ). These parameters are chosen so that the average of pairwise Kendall's taus (also called the coefficient of agreement [31]) of the corresponding $d$-variate extreme-value copula $C_{A}$ is $1 / 2$. The Archimedean generator is chosen to be Gumbel with $\theta=5 / 3$, which corresponds to Kendall's tau of $2 / 5$ of the corresponding bivariate Archimedean copula $C_{\psi}$. The left panel in Figure 3 shows the IRAE for various sample sizes when $d=4$. It is clear that the performance of the estimator improves with sample size, but also that it depends on the Pickands dependence function; the CFG-type estimator performs worse when $A$ is LG. Other dimensions and Archimedean generators led to the same conclusions. It is worth noting that the asymmetric Pickands dependence function NSD does not lead to better or worse results overall.

The right panel of Figure 3 shows the effect of dimension. Unsurprisingly, the performance of the CFG-type estimator deteriorates with $d$. The choice of $A$ has an effect; the latter is most pronounced when $d=4$, although this may be merely due to the choice of parameters. Again, the same pattern was observed for other sample sizes and Archimedean generators. We also tried the DSM Pickands dependence function, which does not satisfy Condition 4.3, because it is not differentiable everywhere. The performance of the CFG-type estimator remained essentially unaffected by this choice of $A$; viz. Tables D7-D9 in the Appendix. This is comforting, because Condition 4.3 is virtually impossible to verify from data.

Our next aim was to study the effect of dependence. We restricted ourselves to the LG Pickands dependence function; in that case, $C_{\psi, A}$ is exchangeable and measuring dependence can be reduced to the bivariate setting. The first study we conducted focused on Kendall's tau. For a bivariate Archimax copula $C_{\psi, A}$, let $\tau_{\psi, A}$ denote its Kendall's tau $\tau\left(C_{\psi, A}\right)$; let also $\tau(A)=\tau\left(C_{A}\right)$ and $\tau(\psi)=\tau\left(C_{\psi}\right)$ denote Kendall's tau of the corresponding bivariate extreme- 

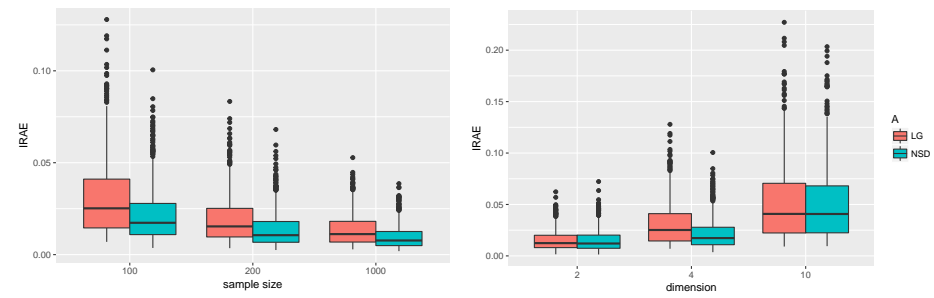

Figure 3: Boxplots of IRAE of $A_{n, c}^{\mathrm{CFG}}$ when $d=4$ and $n \in\{200,500,1000\}$ (left), and when $d \in\{2,4,10\}$ and $n=200$ (right). The Pickands dependence functions are LG (red) and NSD (blue) with coefficient of agreement 1/2; the Archimedean generator is Gumbel with $\theta=5 / 3$.
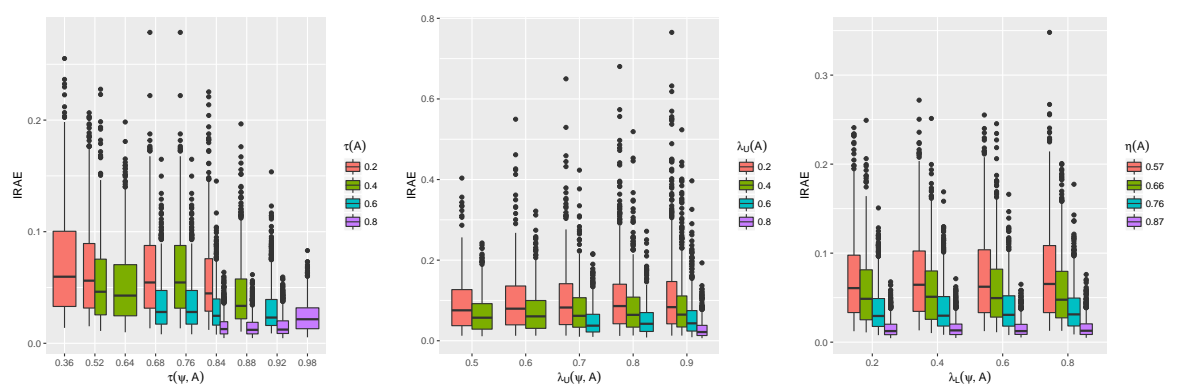

Figure 4: Boxplots of IRAE of $A_{n, c}^{\mathrm{CFG}}$ when $n=200, d=10$ and the Pickands dependence function is $\mathbf{L G}$ for all panels. The Archimedean generators are Frank (left), Joe (middle) and Clayton (right). In the right panel, $\eta_{L}(A)=$ $1 /\{2 A(1 / 2)\}=2^{-1 / \rho}$ is the lower tail dependence index of [34].

value and Archimedean copula, respectively. From [8],

$$
\tau_{\psi, A}=\tau(\psi)+\tau(A)-\tau(\psi) \tau(A) .
$$

The left panel in Figure 4 shows the IRAE of the CFG-type estimator for various values of $\tau_{\psi, A}$ and $\tau(A)$ when $n=200$ and $d=10$. The observed trend is that for a fixed $\tau_{\psi, A}$, an increase in $\tau(A)$, which implies a decrease in $\tau(\psi)$, results in lower IRAE. There is also a performance gain as $\tau_{\psi, A}$ increases. Conclusions for other Archimedean generators, dimensions and sample sizes are the same; viz. Tables D10-D12 in the Appendix.

The second study focused on the effect of upper tail dependence as measured by the upper tail dependence coefficient $\lambda_{U}$ of [29]. For a bivariate Archimax copula $C_{\psi, A}$ whose generator $\psi$ satisfies Condition 4.1 ,

$$
\lambda_{U}\left(C_{\psi, A}\right)=2-\{2 A(1 / 2)\}^{\frac{1}{m}} .
$$

In the middle panel of Figure 4 the Pickands dependence function is again LG with parameter $\varrho$, so that $A(1 / 2)=2^{1 / \varrho-1}$, and the Archimedean generator is 
Joe with parameter $\theta=m$. Consequently, various values of $\lambda_{U}\left(C_{\psi, A}\right)$ can be obtained by varying $\varrho$ and $\theta$. There is a noticeable decrease in IRAE when the contribution of $A$ to $\lambda_{U}\left(C_{\psi, A}\right)$ increases, and a slight increase in error for a fixed $\theta$ when $\lambda_{U}\left(C_{\psi, A}\right)$ increases. The same pattern was observed for other choices of $n$ and $d$; viz. Table D13 in the Appendix.

The last study focused on the effect of lower tail dependence as measured by the lower tail dependence coefficient $\lambda_{L}$ of [29]. For a bivariate Archimax copula $C_{\psi, A}$ whose generator $\psi$ satisfies Condition 4.2 (a),

$$
\lambda_{L}\left(C_{\psi, A}\right)=\{2 A(1 / 2)\}^{-s} .
$$

Again, we considered the LG Pickands dependence function. As the Archimedean generator we choose the Clayton generator, which is such that $s=$ $1 / \theta$. The right panel of Figure 4 shows that the effects of lower and upper tail dependence are similar: an increase in the contribution of $A$ to $\lambda_{L}$ leads to lower IRAE. There is also a slight decrease in performance when $\theta$ is fixed and $\lambda_{L}\left(C_{\psi, A}\right)$ increases. The same pattern was observed for other choices of $n$ and $d$; viz. Table D14 in the Appendix.

\section{Estimation of the distortion function}

Sections 3 to 5 focused on the nonparametric estimation of the stdf under the assumption that the distortion parameter $\psi$ is known. Before these estimators can be used however, $\psi$ needs to be estimated without the knowledge of $\ell$. To do so, assume that $\psi \in \Psi=\left\{\psi_{\theta}, \theta \in \Theta\right\}$. Recall that under the assumptions of Proposition 2.1, $\theta$ and $\ell$ are then identifiable. In this section, we propose a simple moment-based procedure for the most common scenario where $\Theta \subseteq \mathbb{R}$.

First consider an arbitrary bivariate copula $C$ and a pair $\left(U_{1}, U_{2}\right) \sim C$. The distribution function $K_{C}$ of the random variable $W_{C}=C\left(U_{1}, U_{2}\right)$ is called the Kendall distribution [40]. If $C=C_{\psi, A}$ is Archimax, it is known from (13) in [9] that for any $w \in[0,1], K_{C_{\psi, A}}(w)=K_{C_{\psi}}(w)+\phi(w) / \phi^{\prime}(w) \tau(A)$, where $\tau(A)$ is Kendall's tau of $C_{A}$. From this it is easily seen that for any $k \in \mathbb{N}$, the $k$-th moment of $W_{C_{\psi, A}}$ satisfies

$$
m_{k}=\mathrm{E}\left(W_{C_{\psi, A}}^{k}\right)=\frac{\tau(A)}{k+1}+\{1-\tau(A)\} \mathrm{E}\left(W_{C_{\psi}}^{k}\right) .
$$

Equations (6.1) for $k=1$ and $k=2$ then lead to the following identity:

$$
\frac{1-2 \mathrm{E}\left(W_{C_{\psi}}\right)}{1-3 \mathrm{E}\left(W_{C_{\psi}}^{2}\right)}=\frac{1-2 m_{1}}{1-3 m_{2}}
$$

The left-hand side depends only on the Archimedean generator and is thus a function of $\theta$, say $f$. Assuming that $\psi$ is twice differentiable, Theorem 4.3 .4 in [40] and partial integration yield that for any $\theta \in \Theta$,

$$
f(\theta)=\frac{1-2 \mathrm{E}\left(W_{C_{\psi_{\theta}}}\right)}{1-3 \mathrm{E}\left(W_{C_{\psi_{\theta}}}^{2}\right)}=\frac{\int_{0}^{x_{\psi_{\theta}}} x\left\{\psi_{\theta}^{(1)}(x)\right\}^{2} d x}{3 \int_{0}^{x_{\psi_{\theta}}} x \psi_{\theta}(x)\left\{\psi_{\theta}^{(1)}(x)\right\}^{2} d x} .
$$


The following example provides explicit expressions for $f$ for three families of generators; in each case, $f$ is strictly monotone in $\theta$.

Example 6.1. For the Clayton generator given, for any $x \geq 0$, by $\psi_{\theta}(x)=$ $(1+\theta x)_{+}^{-1 / \theta}$ where $\theta \geq-1$ and by $\exp (-x)$ when $\theta=0, E\left(W_{\psi_{\theta}}^{k}\right)=(\theta+$ $1) /\{(k+1)(\theta+k+1)\}$ for any integer $k \geq 1$. Consequently,

$$
f(\theta)=\frac{\theta+3}{2(\theta+2)}
$$

Next, consider the Genest-Ghoudi family [17] whose generator is given, for any $x \in[0,1]$, by $\psi_{\theta}(x)=\left(1-x^{\theta}\right)^{1 / \theta}$ for $\theta \in(0,1]$. Here, $E\left(W_{\psi_{\theta}}^{k}\right)=(1-$ $\theta) /(k+1-\theta)$, for any integer $k \geq 1$. Hence, for any $\theta \in(0,1]$,

$$
f(\theta)=\frac{3-\theta}{4-2 \theta} .
$$

Finally, for the bivariate Frank family with parameter $\theta \in \mathbb{R}$, the generator is given, for any $x \geq 0$, by $\psi_{\theta}(x)=-(1 / \theta) \ln \left\{1+e^{-x}\left(e^{-\theta}-1\right)\right\}$. For $j \in \mathbb{N}$, let $D_{j}(\theta)=\left(j / \theta^{j}\right) \int_{0}^{\theta}\left(t^{j}\right) /\left(e^{t}-1\right) d t$ denote the Debye function [1, Chapter 27]. Here, (6.3) yields after some algebra that for any $\theta \in \mathbb{R}$,

$$
f(\theta)=\frac{4 \theta-4 \theta D_{1}(\theta)}{3\left\{2 \theta-\theta D_{2}(\theta)+4 D_{1}(\theta)-4\right\}} .
$$

If $f$ is one-to-one, as was the case in Example 6.1, (6.2) can be used to construct an estimator of $\theta$. Following [5], define $I_{i j}=\mathbb{1}\left(X_{i} \leq X_{j}, Y_{i} \leq Y_{j}\right)$ for all $i, j \in\{1, \ldots, n\}$ and set

$$
\hat{m}_{1}=\frac{1}{n(n-1)} \sum_{i \neq j} I_{i j}, \quad \hat{m}_{2}=\frac{1}{n(n-1)(n-2)} \sum_{i \neq j \neq k} I_{i j} I_{k j} .
$$

As $\hat{m}_{1}$ and $\hat{m}_{2}$ are $U$-statistics with square integrable kernels, the results of [5] imply that $\sqrt{n}\left\{\left(\hat{m}_{1}, \hat{m}_{2}\right)-\left(\mathrm{E}\left(W_{C}\right), \mathrm{E}\left(W_{C}^{2}\right)\right)\right\} \rightsquigarrow \mathcal{N}(\mathbf{0}, \Sigma)$ as $n \rightarrow \infty$; the entries of $\Sigma$ are given in Proposition 2 therein.

Next, provided $f$ has an inverse $f^{\leftarrow}$, define $h: \mathbb{R}^{2} \rightarrow \mathbb{R}$ by

$$
h\left(m_{1}, m_{2}\right)=f^{\leftarrow}\left(\frac{1-2 m_{1}}{1-3 m_{2}}\right)
$$

and set $\hat{\theta}=h\left(\hat{m}_{1}, \hat{m}_{2}\right)$. Assuming $h$ has continuous partial derivatives that are non-zero at $\left(m_{1}, m_{2}\right)$ and using the delta method, one gets that $\sqrt{n}(\hat{\theta}-\theta) \rightsquigarrow$ $\mathcal{N}\left(0, J_{h}\left(m_{1}, m_{2}\right) \Sigma J_{h}\left(m_{1}, m_{2}\right)^{T}\right)$, where $J_{h}$ is the $2 \times 1$ Jacobian matrix of $h$. Consistent plug-in estimators of the entries of $\Sigma$ are provided in [5]. For small $n$, the calculations of [5] can also be used to compute and estimate the finite sample variance-covariance matrix of $\left(\hat{m}_{1}, \hat{m}_{2}\right)$. 
Example 6.2. For the Clayton family, $\hat{\theta}=S_{n} / R_{n}$, where

$$
S_{n}=8 \hat{m}_{1}-9 \hat{m}_{2}-1, \quad R_{n}=1-4 \hat{m}_{1}+3 \hat{m}_{2} .
$$

Letting $S=8 m_{1}-9 m_{2}-1$ and $R=1-4 m_{1}+3 m_{2}$,

$$
\sqrt{n}(\hat{\theta}-\theta)=\sqrt{n}\left\{h\left(\hat{m}_{1}, \hat{m}_{2}\right)-h\left(m_{1}, m_{2}\right)\right\} \rightsquigarrow \mathcal{N}\left(0, \sigma^{2}\right)
$$

with the asymptotic variance

$$
\begin{aligned}
\sigma^{2}= & \frac{1}{R^{4}}\left\{R^{2}\left(64 \Sigma_{11}+81 \Sigma_{22}-144 \Sigma_{12}\right)\right. \\
& \left.+S^{2}\left(16 \Sigma_{11}+9 \Sigma_{22}-24 \Sigma_{12}\right)-2 R S\left(32 \Sigma_{11}-27 \Sigma_{22}+50 \Sigma_{12}\right)\right\} .
\end{aligned}
$$

Note that the numerator $S_{n}$ in (6.4) is the quantity on which the test for bivariate extreme-value dependence of [21] is based. These authors showed that when $C$ is extreme-value, $8 E\left(W_{C}\right)-9 E\left(W_{C}^{2}\right)-1=0$. When $\theta=0$, the Clayton generator simplifies to $\psi(t)=e^{-t}$ and $C_{\psi, A}=C_{A}$ is extreme-value.

For the Genest-Ghoudi family, $\hat{\theta}=-S_{n} / R_{n}$, where $S_{n}$ and $R_{n}$ are as in (6.4). Hence $\sqrt{n}(\hat{\theta}-\theta) \rightsquigarrow \mathcal{N}\left(0, \sigma^{2}\right)$, where $\sigma^{2}$ is given by (6.5).

For the bivariate Frank family, the function $f$ is one-to-one but its inverse is not explicit. Therefore, both the estimator and the asymptotic variance are not explicit either. An estimate of $\theta$ can be obtained numerically and its asymptotic variance can be studied via resampling.

In the multivariate case, a generalization of (6.1) does not seem possible. We thus propose to use $\hat{\theta}=[2 /\{d(d-1)\}] \sum_{j<k} \hat{\theta}_{j k}$, where $\hat{\theta}_{j k}$ is the above moment-based estimator of $\theta$ based on the bivariate sample $\left(X_{1 j}, X_{1 k}\right), \ldots$, $\left(X_{n j}, X_{n k}\right)$. A heuristic approach for checking whether averaging the pairwise estimates is reasonable is presented in the following section.

\section{Data application}

In this section, the practical usefulness of the proposed estimation procedure for Archimax copula models is illustrated in the context of flood monitoring. The data is a trivariate sample of daily precipitation amounts in French Brittany from 1976 to 2016 provided by Météo France. To avoid seasonality, the series is restricted to September to February, during which most extreme events occur. The position of the three stations Belle-Île, Groix, and Lorient is shown in the left panel of Figure 5.

To remove time dependence, and since our primary focus is on extreme precipitation, we considered monthly maxima at each station, totalling $240 \mathrm{ob}-$ servations. Blocking the data by months also eliminates ties; in particular, it avoids the large number of zeros in the sample of daily maxima. This series shows no departures from stationarity; the Ljung and Box-Pierce tests do not

reject the hypothesis of temporal independence except at Groix, where there is 
slight evidence of dependence at lags 1 and 2. As the asymptotic results hold for alpha-mixing sequences, time dependence is allowed.

The pairs of the normalized component-wise ranks of monthly maxima are displayed in the right panel of Figure 5 . These plots show strong correlation between Lorient and Groix, which is not surprising given their geographical proximity. Also apparent is asymmetry between Belle-Île on the one hand and both Lorient and Groix on the other, in the sense that large precipitation amounts at Groix correspond to large precipitation amounts at Belle-Île, but not necessarily vice versa, and similarly for Lorient.

Because the data at hand are monthly maxima, one might first think of fitting an extreme-value copula model. However the test of [32] clearly rejects the hypothesis of extreme-value dependence ( $\mathrm{p}$-val. $\approx 5 \times 10^{-5}$ ). This may be explained by the presence of lower-tail dependence, which manifests itself by the clumping of points in the bottom-left corner of the rankplots in the right panel of Figure 5. The empirical estimates of the tail probabilities plotted against $q$ in the bottom row of Figure 6 also indicate that all pairwise lower tail dependence coefficients are likely greater than 0 . This phenomenon is not present in multivariate extreme-value distributions, whose all pairwise lower tail dependence coefficients are equal to 0. Archimax copula models advocated in this paper may on the other hand capture lower-tail as well as extremal dependence. The Clayton-Archimax model may be particularly well suited. The latter assumes continuous marginals and an Archimax copula of the form $C_{\psi_{\theta}, A}$, where $A$ is an arbitrary Pickands dependence function and $\psi_{\theta}$ is the Clayton generator given for all $t \geq 0$ by $\psi_{\theta}(t)=(1+\theta t)^{-1 / \theta}$, where $\theta \in[-1 /(d-1), \infty)$ is an unknown parameter. Because $\psi_{\theta}$ for any $\theta>0$ satisfies Condition 4.2 (a) with $s=1 / \theta$, the lower tail dependence coefficient equals $\{2 A(1 / 2)\}^{-1 / \theta}$. Furthermore, Condition 4.1 holds with $m=1$, so that $C_{\psi_{\theta}, A}$ is in the domain
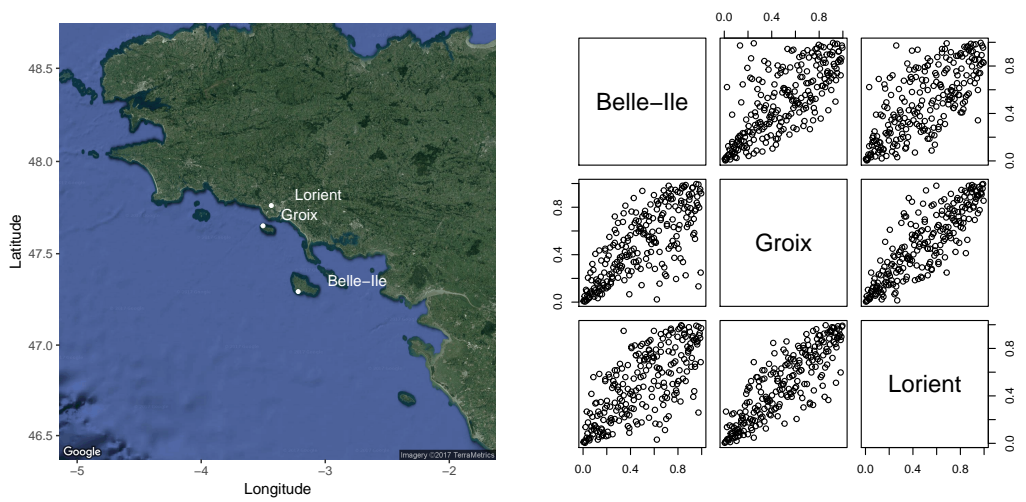

Figure 5: Satellite map of French Brittany, showing the sites Belle-Île, Groix, and Lorient (left). Rankplots of monthly maximum precipitation for the months of September to February, from 1976 to 2016 (right). 


\begin{tabular}{rcccc}
\hline & $\hat{\theta}$ & $90 \%$ C.I. & $\tau\left(C_{\hat{\psi}, \hat{A}}\right)$ & $\tau_{n}$ \\
\hline Belle-Île \& Groix & 1.58 & $(0.77,2.39)$ & 0.54 & 0.56 \\
Belle-Île \& Lorient & 1.08 & $(0.49,1.67)$ & 0.51 & 0.52 \\
Groix \& Lorient & 1.27 & $(0.54,1.99)$ & 0.64 & 0.67 \\
\hline
\end{tabular}

Table 1: Pairwise estimates $\hat{\theta}$ along with $90 \%$ asymptotic confidence intervals in the Clayton-Archimax model, model-based estimates $\tau\left(C_{\hat{\psi}, \hat{A}}\right)$ of pairwise Kendall's tau in the Clayton-Archimax model, and empirical estimates $\tau_{n}$ of pairwise Kendall's tau.

of attraction of the extreme-value copula $C_{A}$. The Clayton-Archimax model is fitted to the data in Section 7.1; comparisons with other estimators of the limiting $A$ are considered in Section 7.2.

\subsection{Fitting the Clayton-Archimax model}

We begin by estimating the Clayton distortion using the moment-based method presented in Section 6. The pairwise estimates of $\theta$ are given in Table 1, along with $90 \%$ confidence intervals. Because these intervals overlap, there is no evidence against a trivariate Clayton-Archimax model with a common value of $\theta$. The latter is estimated by the average of the pairwise estimates to be $\hat{\theta}=1.31$.

The next step consists of estimating $A$. We use the CFG-type estimator $A_{n, c}^{\mathrm{CFG}}$ given in (3.5) with $\psi$ replaced by $\psi_{\hat{\theta}}$. The Pickands-type estimator is not well suited here, because for the estimated value of $\theta, s \approx 0.76<2$, so that the requirements of Theorem 4.2 are likely violated. On the other hand, assuming that Condition 4.3 holds, the hypotheses of Theorem 4.1 are fulfilled. However, the latter theorem is only the first step in the assessment of the limiting behaviour of $A_{n, c}^{\mathrm{CFG}}$ because the Archimedean generator is estimated parametrically rather than treated as known. To establish the limiting behavior of $\mathbb{A}_{n}^{C F G}$ under this more general setup seems very complex however. Instead, we run a pilot simulation which is detailed in Section 7.2 and the results of which are shown in Figure 8. The boxplots $\mathrm{AXC}(1)$ and $\mathrm{AXC}(2)$ summarize the IRAE when $\psi$ is known and estimated parametrically, respectively. Unsurprisingly, parameter uncertainty increases the variability of the estimator; this is also confirmed by our preliminary calculations which show that the limiting process of $\mathbb{A}_{n}^{C F G}$ will have an additional term involving the asymptotic distribution of $\hat{\theta}$. Nonetheless, we conjecture that $\mathbb{A}_{n}^{C F G}$ converges to a centered Gaussian process as $n \rightarrow \infty$.

A contour plot of $A_{n, c}^{\mathrm{CFG}}$ is shown in the left panel of Figure 7. The contour levels of $A_{n, c}^{\mathrm{CFG}}$ show a clear global asymmetry, but axial symmetry with respect to Belle-Île. This pattern corroborates what was seen on the rankplots in Figure 5. This asymmetry may be explained by the fact that Belle-Ile is located far off shore. This can lead to strong localized rainfall which does not affect the stations at Groix and Lorient. Although Groix is also an island, it 
lies much closer to the coast, and is hence not affected by the localized rainfall phenomenon. Furthermore, it can also be seen from pressure maps and radar images that heavy rainfall at Groix and Lorient is mainly due to large-scale weather systems that affect Belle-Île as well.

Finally, we check the fit of the Clayton-Archimax model. Because $A_{n, c}^{\mathrm{CFG}}$ is nonparametric, no existing formal goodness-of-fit test for copula models can be used. However, the contours of the fitted trivariate Clayton-Archimax copula seem fairly close to the empirical copula, as evidenced by the bottom panel of Figure 6 . We also compared various sample dependence measures to their model estimates. To assess the fit in the tails, we consider each pair of stations $j \neq k$, say. Following [12], we plot the empirical estimates of

$$
\begin{aligned}
& \chi_{U}(q)=2-\frac{\log \operatorname{Pr}\left\{F_{j}\left(X_{j}\right)<q, F_{k}\left(X_{k}\right)<q\right\}}{\log (q)} \\
& \chi_{L}(q)=2-\frac{\log \operatorname{Pr}\left\{F_{j}\left(X_{j}\right)>1-q, F_{k}\left(X_{k}\right)>1-q\right\}}{\log (q)}
\end{aligned}
$$

against $q$ together with the model-based estimates of the lower and upper tail dependence coefficients $\lambda_{L}$ and $\lambda_{U}$ for that pair, respectively. To compute the latter, we use that in a bivariate Clayton-Archimax model,

$$
\lambda_{L}=\lim _{q \rightarrow 1} \chi_{L}(q)=\{2 A(1 / 2)\}^{-1 / \theta}, \quad \lambda_{U}=\lim _{q \rightarrow 1} \chi_{U}(q)=2-2 A(1 / 2) .
$$

The top two panels of Figure 6 show that the model-based estimates approximate the empirical probabilities quite nicely when $q \rightarrow 1$, which indicates a good fit in the tails. The contour plots of the empirical copula and the fitted ClaytonArchimax model displayed in the bottom panel of the same Figure match nicely as well. Finally, we compared empirical estimates of pairwise Kendall's tau with model-based estimates. To compute the latter, we used (5.2) with $\tau_{\psi}=\theta /(\theta+2)$ and $\tau(A)=\int_{0}^{1}[\{t(1-t)\} / A(t)] d A^{\prime}(t)$, and approximated the integral in the expression for $\tau(A)$ with finite differences. Table 1 shows that the empirical and model-based estimates are very close. Overall, the fit of the Clayton-Archimax model seems adequate, and allows to model the dependence in this trivariate precipitation dataset, not only in extremes, but also in a medium size regime.

\subsection{Comparison with other estimators of $A$}

If the objective is to specifically assess the joint risk of extreme precipitation, then the estimation of the Pickands dependence function $A$ of the extremevalue attractor of the distribution of the monthly maxima at the three stations is of interest. Because the Clayton-Archimax copula $C_{\psi, A}$ is in the domain of attraction of $C_{A}$, the estimator $A_{n, c}^{\mathrm{CFG}}$ calculated in the preceding section is also an estimate of the limiting Pickands dependence function. As such, it can be compared to other nonparametric estimators considered in the literature.

The first idea would be to block the data by seasons and consider the maxima over the period from September to February. This reduces the sample size to 

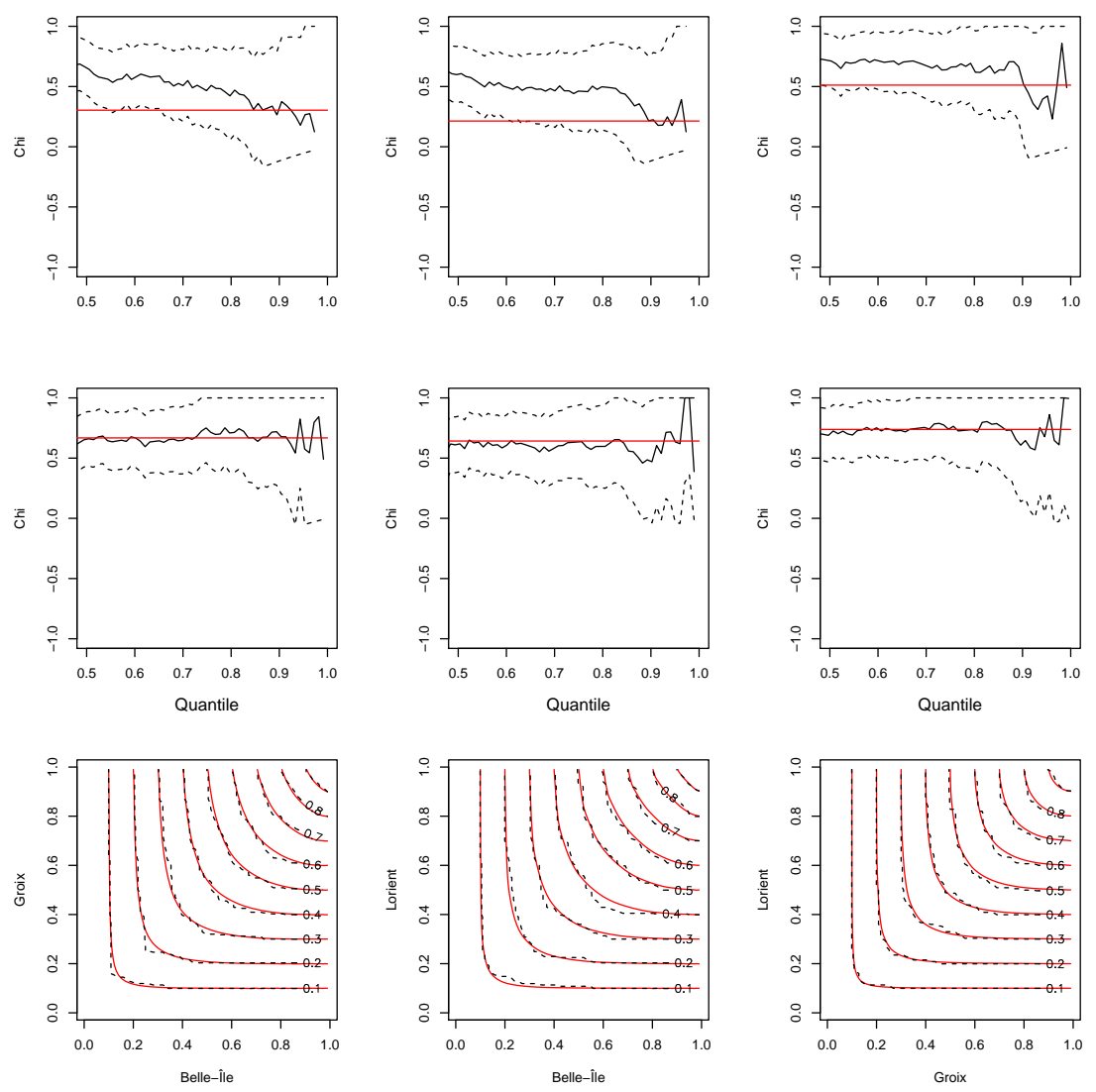

Figure 6: Empirical estimates of $\chi_{U}(q)$ (top) and $\chi_{L}(q)$ (middle) plotted against $q$ (Quantile) along with $95 \%$ confidence bands (black). The red lines indicate the model-based estimates of $\lambda_{U}$ (top) and $\lambda_{L}$ (middle). Contour plots (bottom) of the empirical copula (black dashed) and the fitted Clayton-Archimax copula (red). The plots correspond to Belle-Île \& Groix (left), Belle-Île \& Lorient (middle), and Groix \& Lorient (right).

$n=40$, but the hypothesis of extreme-value dependence is no longer rejected by the test of $[32]$ (p-val. $\approx 0.43$ ). Consequently, the multivariate rank-based CFG estimator of [23] can be used. Another option would be to use nonparametric estimators of $A$ that only assume that the underlying copula is in the domain of attraction of $C_{A}$. We consider the FHM and EKS estimators of [16] and [13], respectively. More precisely, the FHM estimator is denoted as $\stackrel{\circ}{L}_{\text {agg }}$ in [16, Section 5.1], built from equation (15) therein, and its tuning parameters are $\kappa_{n}=239, a=0.8, r=0.8, k_{\rho}=237$. The bias-corrected EKS estimator is denoted $\bar{\ell}_{n, k, k_{1}}$ and its parameters were set to the default choices from the $\mathrm{R}$ package tailDepFun. 

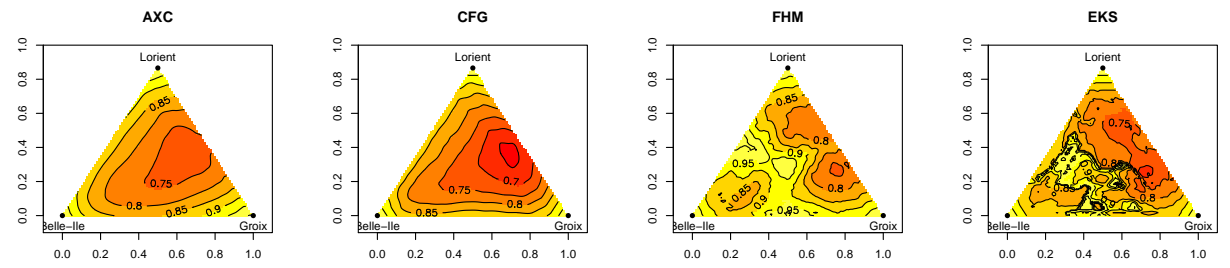

Figure 7: AXC: CFG-type estimator $A_{n, c}^{\mathrm{CFG}}$ based on monthly maxima and the Clayton-Archimax model. CFG: Rank-based CFG estimator of [22] based on seasonal maxima. FHM and EKS: Estimators of [16] and [13] based on monthly maxima.

The three competing estimators CFG, FHM, and EKS are displayed in Figure 7 along with $A_{n, c}^{\mathrm{CFG}}$ from Section 7.1. The contours of the CFG estimator are rougher, which is not surprising given that it is based on 40 observations. Although we expect this estimator to be more variable because it is based on a smaller sample, it is comforting that it shows a similar pattern as $A_{n, c}^{\mathrm{CFG}}$; this further confirms that the Clayton-Archimax model is adequate for the data at hand. The contours of the FHM and EKS estimators are much more irregular which makes the plots difficult to interpret.

To compare these estimators further, we run a pilot simulation study mimicking the data. We generated $N=1000$ samples of size $n=240$ from a trivariate Clayton-Archimax copula with $\theta=1.31$ and the scaled negative extremal Dirichlet Pickands dependence function parameters $\boldsymbol{\alpha}=(1,2,3)$ and $\rho=0.9$ whose shape roughly resembles $A_{n, c}^{\mathrm{CFG}}$ (see the left panel of Figure 8 ). For each sample, we estimated $A$ by: (i) the CFG-type estimator from (3.5) assuming that $\psi$ is known; (ii) the CFG-type estimator from (3.5) with $\theta$ estimated by the moment estimator $\hat{\theta}$ from Section 7.1; (iii) the CFG estimator of [22] based on block maxima with 40 blocks; (iv) the FHM estimator of [16]; (v) the EKS estimator of [13]. The boxplots of the IRAE are shown in Figure 8. Even if $\psi$ is estimated by $\psi_{\hat{\theta}}, A_{n, c}^{\mathrm{CFG}}$ is clearly superior to the CFG, FHM and EKS estimators particularly in terms of bias.

To sum up, this application on precipitation data demonstrates the feasibility of the proposed inference techniques but more importantly illustrates the potential of Archimax copulas to model joint risk in subasymptotic settings. Since the max domain of attraction of Archimax copulas is known, one can check the performance of the latter model by comparing it to models using the max-stable assumption. In this particular data application, the Archimax model accurately captures the bulk and both tails of medium to high precipitation observations. Performance at extreme levels is no doubt also due to the fact that the studied weather stations are located in a relatively small area. To model extremes over larger spatial scales however, more flexible models than those studied herein are required in order to capture asymptotic independence, 
as noted e.g. by [28] and [47].
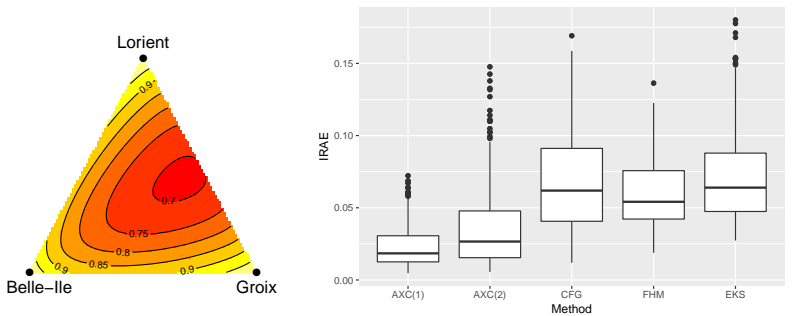

Figure 8: Left: Negative scaled extremal Dirichlet (NSD) Pickands dependence function $A$ with parameters $\boldsymbol{\alpha}=(1,2,3)$ and $\rho=0.9$. Right: Boxplots of $\operatorname{IRAE}\left(\hat{A}_{n}\right)$ based on $N=1000$ samples of size $n=240$ from a 3-variate ClaytonArchimax copula $C_{\psi_{\theta}, A}$ with $\theta=1.31$ and the scaled negative extremal Dirichlet $A$ with parameters $\boldsymbol{\alpha}=(1,2,3)$ and $\rho=0.9$. AXC(1): the CFG-type estimator from (3.5) assuming that $\psi$ is known; $\mathrm{AXC}(2)$ : the CFG-type estimator from (3.5) with $\theta$ estimated by the moment estimator $\hat{\theta}$ from Section 7.1 ; CFG: the CFG estimator of [22] based on block maxima with 40 blocks; FHM: the estimator of [16]; EKS: the estimator of [13].

\section{Acknowledgments}

The authors would like to thank Météo France for kindly providing the data, and in particular Maxime Taillardat for numerous fruitful discussions and also for the convenient format of the data. This work was partially supported by the LABEX MILYON (ANR-10-LABX-0070) of Université de Lyon, within the program "Investissements d'Avenir" (ANR-11-IDEX- 0007) operated by the French National Research Agency (ANR), further by an individual operating grant from the Natural Sciences and Engineering Research Council of Canada (RGPIN2015-06801), and team grants from the Fonds de recherche du Québec - Nature et technologies (2015-PR-183236) and from the Canadian Statistical Sciences Institutes. We thank the $\mathrm{R}$ project that provides free material, and especially Marius Hofert and Martin Maechler, the authors of the $\mathrm{R}$ package simsalapar for their useful package which greatly facilitates simulation studies.

\section{Proofs from Section 2}

Proof of Lemma 2.1. For all $\boldsymbol{u} \in[0,1]^{d}, \ell_{1}\left\{\psi^{-1}(\boldsymbol{u})\right\}=\ell_{2}\left\{\psi^{-1}(\boldsymbol{u})\right\}$, and since $\psi^{-1}$ is one-to-one, $\ell_{1}(\boldsymbol{x})=\ell_{2}(\boldsymbol{x})$ for all $\boldsymbol{x} \in \mathbb{R}_{+}^{d}$.

Proof of Lemma 2.2. If $\ell(\boldsymbol{x})=\ell_{M}(\boldsymbol{x})=\max \left\{x_{1}, \ldots, x_{d}\right\}$ for all $\boldsymbol{x} \in \mathbb{R}_{+}^{d}$, then regardless of $\psi_{1}$ and $\psi_{2}$, we have that $C_{1}=C_{2}=C_{M}$, the copula corresponding to the Fréchet-Hoeffding upper bound which describes comonotonic variables. 
Now suppose that $\ell \neq \ell_{M}$. Then it is clear that $C_{k} \neq C_{M}$ for both $k \in\{1,2\}$. Indeed, fix $k \in\{1,2\}$. Note that $\ell(\boldsymbol{x})>\ell_{M}(\boldsymbol{x})$ for some $\boldsymbol{x} \in \mathbb{R}_{+}^{d}$. By the homogeneity of $\ell$, there also exists an $x \in \mathbb{R}_{+}^{d}$ such that $0<\psi_{k}\{\ell(\boldsymbol{x})\}<$ $\psi_{k}\left\{\ell_{M}(\boldsymbol{x})\right\}$. Therefore, $C_{k}(\boldsymbol{u})=\psi_{k} \circ \ell\left\{\psi_{k}^{-1}(\boldsymbol{u})\right\}<\psi_{k} \circ \ell_{M}\left\{\psi_{k}^{-1}(\boldsymbol{u})\right\}=C_{M}(\boldsymbol{u})$ for $\boldsymbol{u}=\psi_{k}(\boldsymbol{x})$. Consequently, there exists at least one pair $i, j \in\{1, \ldots, d\}$, $i<j$, such that the bivariate margin of $C_{k}$, given, for all $u_{i}, u_{j} \in[0,1]$, by

$$
C_{k}^{(i j)}\left(u_{i}, u_{j}\right):=C_{k}\left(1, \ldots, 1, u_{i}, 1, \ldots, 1, u_{j}, 1, \ldots, 1\right)
$$

is not the Fréchet-Hoeffding upper bound copula. Next note that for all $u_{i}, u_{j} \in$ $[0,1]$,

$$
\begin{aligned}
C_{1}^{(i j)}\left(u_{i}, u_{j}\right) & =\psi_{1} \circ \ell^{(i j)}\left\{\psi_{1}^{-1}\left(u_{i}\right), \psi_{1}^{-1}\left(u_{j}\right)\right\} \\
& =\psi_{2} \circ \ell^{(i j)}\left\{\psi_{2}^{-1}\left(u_{i}\right), \psi_{2}^{-1}\left(u_{j}\right)\right\}=C_{2}^{(i j)}\left(u_{i}, u_{j}\right),
\end{aligned}
$$

where $\ell^{(i j)}$ denotes the bivariate margin of $\ell$, given, for all $x_{i}, x_{j} \in \mathbb{R}_{+}$, by

$$
\ell^{(i j)}\left(x_{i}, x_{j}\right):=\ell\left(0, \ldots, 0, x_{i}, 0, \ldots, 0, x_{j}, 0, \ldots, 0\right) .
$$

Therefore, $C_{k}^{(i j)}, k \in\{1,2\}$ are bivariate Archimax. According to Equation (13) of [9], they have the following Kendall's function for $w \in[0,1]$,

$$
K_{k}(w)=\tau_{\ell^{(i j)}} w+\left(1-\tau_{\ell^{(i j)}}\right) K_{\psi_{k}}(w),
$$

where $\tau_{\ell^{(i j)}}$ is the Kendall's tau of the extreme-value copula $C_{\ell^{(i j)}}$ and $K_{\psi_{k}}(w)$ is the Kendall's function of the bivariate Archimedean copula $C_{\psi_{k}}$. Since $\ell^{(i j)} \neq$ $\ell_{M}$, we know that $\tau_{\ell^{(i j)}}<1$ and thus that $K_{\psi_{1}}(w)=K_{\psi_{2}}(w)$. From [20] and [18], it follows that $C_{\psi_{1}}=C_{\psi_{2}}$. By the identifiability of Archimedean copulas, this yields the equality of $\psi_{1}$ and $\psi_{2}$ up to scaling (see for example Chapter 4 of [40]).

Proof of Lemma 2.3. Proof of part (i). Clearly, $\psi_{\beta}$ is a continuous and decreasing function such that $\psi_{\beta}(0)=1$ and $\psi_{\beta}(x) \rightarrow 0$ as $x \rightarrow \infty$. Let $\ell_{\beta}$ be the logistic stdf given, for all $\boldsymbol{x} \in \mathbb{R}_{+}^{d}$ by $\ell_{\beta}\left(x_{1}, \ldots, x_{d}\right)=\left(x_{1}^{1 / \beta}+\cdots+x_{d}^{1 / \beta}\right)^{\beta}$. The Archimax copula $C_{\psi, \ell_{\beta}}$, is a bona-fide copula by Theorem 2.1 in [11]. However, it is easily seen that $C_{\psi, \ell_{\beta}}=C_{\psi_{\beta}}$, where $C_{\psi_{\beta}}$ is the $d$-variate Archimedean copula with generator $\psi_{\beta}$. By Theorem 2.2 in [36], $\psi_{\beta}$ must be $d$-monotone.

Proof of part (ii). Let $\psi_{\beta}$ be the generator of the Gumbel copula given, for all $x \geq 0$, by $\psi_{\beta}(x)=e^{-x^{\beta}}$. Then $\psi_{\beta}$ is a completely monotone Archimedean generator and $1-\psi_{\beta}(1 / x) \in \mathcal{R}_{-\beta}$. By Proposition 6.1 in [11], the $d$-variate Archimax copula $C_{\psi_{\beta}, \ell}$ is in the maximum domain of attraction of the extremevalue copula with stdf $\ell_{\beta}$. Consequently, $\ell_{\beta}$ is a $d$-variate stdf, as claimed.

Proof of Lemma 2.4. Proposition 6.1 in [11] implies that, for all $k \in\{1,2\}$, that $C_{\psi_{k}, \ell_{k}}$ is in the maximum domain of attraction of the extreme-value copula with stdf given, for all $\boldsymbol{x} \in \mathbb{R}_{+}^{d}$, by $\ell_{k}^{1 / m_{k}}\left(\boldsymbol{x}^{m_{k}}\right)$. Because $C_{\psi_{1}, \ell_{1}}=C_{\psi_{2}, \ell_{2}}$ 
by assumption, this implies that for all $\boldsymbol{x} \in \mathbb{R}_{+}^{d}$, it holds that $\ell_{1}^{1 / m_{1}}\left(\boldsymbol{x}^{m_{1}}\right)=$ $\ell_{2}^{1 / m_{2}}\left(\boldsymbol{x}^{m_{2}}\right)$. Hence, for all $\boldsymbol{x} \in \mathbb{R}_{+}^{d}$,

$$
\ell_{1}\left(x_{1}, \ldots, x_{d}\right)=\ell_{2}^{m_{1} / m_{2}}\left(x_{1}^{m_{2} / m_{1}}, \ldots, x_{d}^{m_{2} / m_{1}}\right) .
$$

Thus, for all $\boldsymbol{u} \in[0,1]^{d}$,

$$
C_{\psi_{1}, \ell_{1}}(\boldsymbol{u})=\psi_{1} \circ \ell_{2}^{m_{1} / m_{2}}\left[\left\{\psi_{1}^{-1}\left(u_{1}\right)\right\}^{m_{2} / m_{1}}, \ldots,\left\{\psi_{1}^{-1}\left(u_{d}\right)\right\}^{m_{2} / m_{1}}\right] .
$$

Now set $\psi_{1}^{\star}(t)=\psi_{1}\left(t^{m_{1} / m_{2}}\right)$ for $t \in \mathbb{R}_{+}$and note that $\psi_{1}^{\star}$ is a $d$-monotone Archimedean generator by Lemma 2.3. Therefore, $C_{\psi_{1}, \ell_{1}}=C_{\psi_{1}^{\star}, \ell_{2}}=C_{\psi_{2}, \ell_{2}}$. Given that $\ell_{2} \neq \ell_{M}$ by assumption, the rest of the claim follows from Lemma 2.2.

\section{Smoothness of the Archimax family}

Let first recall the following conditions considered in [44] and [6], featuring the set $V_{d, i}=\left\{\boldsymbol{u} \in[0,1]^{d}: u_{i} \in(0,1)\right\}$.

Condition 9.1. For each $j \in 1, \ldots, d$, the partial derivative $\dot{C}_{j}$ given for all $\boldsymbol{u} \in[0,1]^{d}$ by $\dot{C}_{j}(\boldsymbol{u})=\left(\partial / \partial u_{j}\right) C(\boldsymbol{u})$ exists and is continuous on the set $V_{d, j}$.

Condition 9.2. For every $i, j \in 1, \ldots, d$, the second-order partial derivative $\ddot{C}_{i j}$ given for all $\boldsymbol{u} \in[0,1]^{d}$ by $\ddot{C}_{i j}(\boldsymbol{u})=\left(\partial^{2} / \partial u_{i} \partial u_{j}\right) C(\boldsymbol{u})$ exists and is continuous on the set $V_{d, j} \cap V_{d, i}$, and there exists a constant $K>0$ such that for all $\boldsymbol{u} \in V_{d, j} \cap V_{d, i}$

$$
\left|\ddot{C}_{i j}(\boldsymbol{u})\right| \leq K \min \left\{\frac{1}{u_{i}\left(1-u_{i}\right)}, \frac{1}{u_{j}\left(1-u_{j}\right)}\right\} .
$$

Let $C$ be a $d$-dimensional Archimax copula $C_{\psi, \ell}$. With the notation $\phi(\boldsymbol{u})=$ $\left\{\phi\left(u_{1}\right), \ldots, \phi\left(u_{d}\right)\right\}$, the partial derivatives of $C$ can be computed for each $i, j \in$ $\{1, \ldots, d\}, i \neq j$, as

$$
\begin{gathered}
\dot{C}_{i}(\boldsymbol{u})=\psi^{\prime}[\ell\{\phi(\boldsymbol{u})\}] \dot{\ell}_{i}\{\phi(\boldsymbol{u})\} \phi^{\prime}\left(u_{i}\right) \\
\ddot{C}_{i j}(\boldsymbol{u})=\left(\psi^{\prime \prime}[\ell\{\phi(\boldsymbol{u})\}] \dot{\ell}_{i}\{\phi(\boldsymbol{u})\} \dot{\ell}_{j}\{\phi(\boldsymbol{u})\}+\psi^{\prime}[\ell\{\phi(\boldsymbol{u})\}] \ddot{\ell}_{i j}\{\phi(\boldsymbol{u})\}\right) \\
\times \phi^{\prime}\left(u_{i}\right) \phi^{\prime}\left(u_{j}\right) \\
\ddot{C}_{i i}(\boldsymbol{u})=\left(\psi^{\prime \prime}[\ell\{\phi(\boldsymbol{u})\}]\left[\dot{\ell}_{i}\{\phi(\boldsymbol{u})\}\right]^{2}+\psi^{\prime}[\ell\{\phi(\boldsymbol{u})\}] \ddot{\ell}_{i i}\{\phi(\boldsymbol{u})\}\right) \\
\times\left\{\phi^{\prime}\left(u_{i}\right)\right\}^{2}+\psi^{\prime}[\ell\{\phi(\boldsymbol{u})\}] \dot{\ell}_{i}\{\phi(\boldsymbol{u})\} \phi^{\prime \prime}\left(u_{i}\right)
\end{gathered}
$$




\subsection{Auxiliary results}

Lemma 9.1. Let $\ell$ be a $d$-variate stdf whose first order partial derivatives exist on $\mathbb{R}_{+}^{d}$. Then, for any $i \in\{1, \ldots, d\}$ and $\boldsymbol{x} \in \mathbb{R}_{+}^{d}$, one has $0 \leq \dot{\ell}_{i}(\boldsymbol{x}) \leq 1$.

Proof. Both inequalities can be derived from the properties (a)-(c) in Section 2.1. Fix $i \in\{1, \ldots, d\}$ and $\boldsymbol{x} \in \mathbb{R}_{+}^{d}$. Since $\ell$ is fully $d$-max decreasing, it is increasing in each argument. This yields the first inequality. To show the second inequality, note that properties (a) and (b) imply $\ell\left(0, \ldots, 0, x_{i}, 0, \ldots, 0\right)=x_{i}$, and hence $\dot{\ell}_{i}\left(0, \ldots, 0, x_{i}, 0, \ldots, 0\right)=1$. From property (c), it also follows that $\dot{\ell}_{i}$ is non-increasing in the $j$-th argument for all $j \neq i$. Therefore $\dot{\ell}_{i}(\boldsymbol{x}) \leq$ $\dot{\ell}_{i}\left(0, \ldots, 0, x_{i}, 0, \ldots, 0\right)=1$.

Lemma 9.2. Let $\psi$ be a d-monotone Archimedean generator for some $d \geq 2$ such that $\psi^{\prime}$ exists and is continuous on $(0, \infty)$ when $d=2$. Assume that Conditions 4.1 and 4.2 hold and let $x_{\psi}=\inf \{x \in[0, \infty): \psi(x)=0\}$. Then the function given for any $x \in\left(0, x_{\psi}\right)$ by $f(x)=\psi(x)\{1-\psi(x)\} /\left\{-x \psi^{\prime}(x)\right\}$ is continuous on $\left(0, x_{\psi}\right)$ and has finite limits at 0 and $x_{\psi}$.

Proof. Given that the continuity of $f$ is immediate, it suffices to show that its limits at 0 and $x_{\psi}$ are finite. Because Condition 4.1 holds,

$$
\lim _{x \rightarrow 0} f(x)=\lim _{x \rightarrow \infty} \frac{\psi(1 / x)\{1-\psi(1 / x)\}}{(1 / x)\left\{-\psi^{\prime}(1 / x)\right\}}=m,
$$

where the last equality follows from Equation (12) in [33]. Turning to the limit of $f$ at $x_{\psi}$, three cases have to be distinguished.

Assume first that Condition 4.2 (a) holds. In this case, $x_{\psi}=\infty$ and Equation (7) in [33] implies $\lim _{x \rightarrow \infty} f(x)=1 / s$. Next, assume that Condition 4.2 (b) holds. Because the function given for all $x \in\left(0, x_{\psi}\right)$ by $\psi(x) /\left\{-\psi^{\prime}(x)\right\}$ is an auxiliary function by the calculations in the proof of Theorem 1 (c) on p. 213 in [33], $\lim _{x \rightarrow x_{\psi}} f(x)=0$ by Lemma 3.10.1 [7]. Finally, assuming Condition 4.2 (c), $x_{\psi}<\infty$ and

$$
\begin{aligned}
\lim _{x \rightarrow x_{\psi}} f(x) & =\lim _{x \rightarrow \infty} \frac{\left\{1-\psi\left(x_{\psi}-1 / x\right)\right\} \psi\left(x_{\psi}-1 / x\right)}{-\psi^{\prime}\left(x_{\psi}-1 / x\right)\left(x_{\psi}-1 / x\right)} \\
& =\lim _{x \rightarrow \infty} \frac{x \psi\left(x_{\psi}-1 / x\right)}{-\psi^{\prime}\left(x_{\psi}-1 / x\right)} \frac{(1 / x)\left\{1-\psi\left(x_{\psi}-1 / x\right)\right\}}{x_{\psi}-1 / x}=0,
\end{aligned}
$$

since the first ratio in the last expression tends to $1 /(\alpha+d-1)$ thanks to Condition 4.2 (c) and [33, p. 211].

Lemma 9.3. Let $\psi$ be a d-monotone Archimedean generator for some $d \geq 3$ such that $\psi^{\prime \prime}$ exists and is continuous on $(0, \infty)$. Assume that Conditions 4.1 and 4.2 hold and let $x_{\psi}=\inf \{x \in[0, \infty): \psi(x)=0\}$. Then the function given for any $x \in\left(0, x_{\psi}\right)$ by $f(x)=\psi(x)\{1-\psi(x)\} \psi^{\prime \prime}(x) /\left\{\psi^{\prime}(x)\right\}^{2}$ is continuous on $\left(0, x_{\psi}\right)$ and has finite limits at 0 and $x_{\psi}$. 
Proof. As in the proof of Lemma 9.2, the continuity of $f$ is immediate and hence it suffices to show that its limits at 0 and $x_{\psi}$ are finite. From Condition 4.1 and Equation (12) in [33],

$$
\lim _{x \rightarrow 0} f(x)=\lim _{x \rightarrow \infty} \frac{\psi(1 / x)\{1-\psi(1 / x)\}(1 / x)^{2} \psi^{\prime \prime}(1 / x)}{\left\{-(1 / x) \psi^{\prime}(1 / x)\right\}^{2}}=m-1,
$$

Turning to the limit of $f$ at $x_{\psi}$, three cases have to be distinguished.

Assume first that Condition 4.2 (a) holds. In this case, $x_{\psi}=\infty$ and Equation (7) in [33] implies $\lim _{x \rightarrow \infty} f(x)=(s+1) / s$.

Next, assume that Condition 4.2 (b) holds. By the calculations in the proof of Theorem 1 (c) on p. 213 in [33], the functions given for all $x \in\left(0, x_{\psi}\right)$ by $a_{1}^{*}(x)=\psi(x) /\left\{-\psi^{\prime}(x)\right\}$ and $a_{2}^{*}(x)=-\psi^{\prime}(x) / \psi^{\prime \prime}(x)$ are auxiliary functions that are asymptotically equivalent to the auxiliary function $a$ of $\psi$. Consequently, $a_{1}^{*}(x) / a_{2}^{*}(x) \rightarrow 1$ as $x \rightarrow x_{\psi}$ so that $\lim _{x \rightarrow x_{\psi}} f(x)=1$.

Finally, assuming Condition 4.2 (c), $x_{\psi}<\infty$ and

$$
\begin{aligned}
\lim _{x \rightarrow x_{\psi}} f(x) & =\lim _{x \rightarrow \infty} \frac{\psi\left(x_{\psi}-1 / x\right)}{-(1 / x) \psi^{\prime}\left(x_{\psi}-1 / x\right)} \frac{(1 / x)^{2} \psi^{\prime \prime}\left(x_{\psi}-1 / x\right)}{-(1 / x) \psi^{\prime}\left(x_{\psi}-1 / x\right)}\left\{1-\psi\left(x_{\psi}-1 / x\right)\right\} \\
& =\frac{\alpha+d-2}{\alpha+d-1},
\end{aligned}
$$

where the last equality follows from the calculations on p. 211 in the proof of Theorem 1 (b) in [33].

\subsection{Verification of the smoothness conditions}

Proposition 1. Let $C=C_{\psi, \ell}$ be a d-variate Archimax copula such that $\psi^{\prime}$ exists and is continuous on $(0, \infty)$ when $d=2$, and the first order partial derivatives of $\ell$ exist and are continuous on $\mathbb{R}_{+}^{d}$. Then Condition 9.1 holds.

Proof. Fix $j \in\{1, \ldots, d\}, \boldsymbol{u} \in V_{d, j}$, set $\boldsymbol{x}=\phi(\boldsymbol{u})$ and using (9.2) write

$$
\dot{C}_{j}\{\psi(\boldsymbol{x})\}=\frac{\psi^{\prime}\{\ell(\boldsymbol{x})\} \dot{\ell}_{j}(\boldsymbol{x})}{\psi^{\prime}\left(x_{j}\right)} .
$$

Because $\psi^{\prime}>0$ on $\left(0, \psi^{-1}(0)\right)$, and $\ell(\boldsymbol{x}) \geq x_{j}>0$ on $V_{d, j}$, the assumptions imply that $\dot{C}_{j}$ is continuous on $(0,1]^{d} \cap V_{d, j}$. If $u_{i} \rightarrow 0$ for at least one $i \neq j$, $x_{i} \rightarrow \psi^{-1}(0)$ and $\ell(x) \rightarrow \ell\left(x_{1}, \ldots, x_{i-1}, \psi^{-1}(0), x_{i+1}, \ldots, x_{d}\right) \geq \psi^{-1}(0)$. By Lemma 1 in [48], $\psi^{\prime}(x) \rightarrow 0$ as $x \rightarrow \psi^{-1}(0)$ and if $\psi^{-1}(0)<\infty, \psi^{\prime}(x)=0$ for $x \geq \psi^{-1}(0)$. Consequently, as $x_{i} \rightarrow \psi^{-1}(0), \dot{C}_{j}\{\psi(\boldsymbol{x})\} \rightarrow 0$.

Proposition 2. Let $C=C_{\psi, \ell}$ be a d-variate Archimax copula such that $\psi$ is $k$-monotone for some $k \geq 3$ and $\psi^{\prime \prime}$ exists and is continuous on $(0, \infty)$. If Conditions 4.1, 4.2 (a) and 4.3 hold, or if $-\log (\psi)$ is concave and Conditions 4.1, 4.2 (b) and 4.3 hold, then Condition 9.2 is satisfied. 
Proof. For any $\boldsymbol{u} \in[0,1]^{d}$, set $\boldsymbol{x}=\phi(\boldsymbol{u})$ and for any $i, j \in\{1, \ldots, d\}$, introduce the following terms:

$$
\begin{gathered}
T_{i j, 1}(\boldsymbol{x})=\frac{\psi^{\prime \prime}\{\ell(\boldsymbol{x})\}}{\psi^{\prime}\left(x_{i}\right) \psi^{\prime}\left(x_{j}\right)}, \quad T_{i j, 2}(\boldsymbol{x})=\frac{-M \psi^{\prime}\{\ell(\boldsymbol{x})\}}{\left(x_{i} \vee x_{j}\right) \psi^{\prime}\left(x_{i}\right) \psi^{\prime}\left(x_{j}\right)}, \\
T_{i i, 3}(\boldsymbol{x})=\frac{\psi^{\prime}\{\ell(\boldsymbol{x})\} \psi^{\prime \prime}\left(x_{i}\right)}{\left\{\psi^{\prime}\left(x_{i}\right)\right\}^{3}} .
\end{gathered}
$$

By the $d$-monotonicity of $\psi$, observe first that for $k \in\{1,2,3\}, T_{i j, k} \geq 0$. Now let $x_{\psi}=\inf \{x \in[0, \infty): \psi(x)=0\}$. From (9.3), (9.4), Lemma 9.1, and Condition 4.3 it follows that for any $\boldsymbol{x} \in\left(0, x_{\psi}\right)^{d}$,

$\left|\ddot{C}_{i j}\{\psi(\boldsymbol{x})\}\right| \leq T_{i j, 1}(\boldsymbol{x})+T_{i j, 2}(\boldsymbol{x}), \quad\left|\ddot{C}_{i i}\{\psi(\boldsymbol{x})\}\right| \leq T_{i i, 1}(\boldsymbol{x})+T_{i i, 2}(\boldsymbol{x})+T_{i i, 3}(\boldsymbol{x})$.

Next, note that for any $i \neq j, \ddot{C}_{i j}$ and $\ddot{C}_{i i}$ are continuous on $(0,1]^{d} \cap V_{d, i} \cap V_{d, j}$. The $d$-monotonicity of $\psi$ and Lemma 1 in [48] implies that for $k \in\{1,2\}$, $\psi^{(k)}(x) \rightarrow 0$ as $x \rightarrow x_{\psi}$ and if $x_{\psi}<\infty, \psi^{(k)}(x)=0$ for $x \geq x_{\psi}$. Consequently, for each $k \in\{1,2\}, T_{i j, k}(\boldsymbol{x}) \rightarrow 0$ as $x_{r} \rightarrow x_{\psi}$ for at least one $r \notin\{i, j\}$ and that for each $k \in\{1,2,3\}, T_{i i, k}(\boldsymbol{x}) \rightarrow 0$ as $x_{r} \rightarrow x_{\psi}$ for at least one $r \neq i$. This in turn implies that $\ddot{C}_{i j}\{\psi(\boldsymbol{x})\} \rightarrow 0$ and $\ddot{C}_{i i}\{\psi(\boldsymbol{x})\} \rightarrow 0$ as $x_{r} \rightarrow x_{\psi}$ for at least one $r$ in $\{1, \ldots, d\} \backslash\{i, j\}$ and $\{1, \ldots, d\} \backslash\{i\}$, respectively. Hence for $i \neq j, \ddot{C}_{i j}$ and $\ddot{C}_{i i}$ are continuous on $V_{d, i} \cap V_{d, j}$.

Now introduce the functions given, for any $z_{1}, z_{2} \in\left(0, x_{\psi}\right)$, by

$$
\begin{gathered}
\tilde{T}_{1}\left(z_{1}, z_{2}\right)=\frac{\psi^{\prime \prime}\left\{z_{1} \vee z_{2}\right\}}{\psi^{\prime}\left(z_{1}\right) \psi^{\prime}\left(z_{2}\right)}, \quad \tilde{T}_{2}\left(z_{1}, z_{2}\right)=\frac{-M \psi^{\prime}\left\{z_{1} \vee z_{2}\right\}}{\left(z_{1} \vee z_{2}\right) \psi^{\prime}\left(z_{1}\right) \psi^{\prime}\left(z_{2}\right)}, \\
\tilde{T}_{3}\left(z_{1}\right)=\frac{\psi^{\prime}\left\{z_{1}\right\} \psi^{\prime \prime}\left(z_{1}\right)}{\left\{\psi^{\prime}\left(z_{1}\right)\right\}^{3}}
\end{gathered}
$$

Note first that for $k \in\{1,2,3\}, \tilde{T}_{k} \geq 0$ on its domain. Because $(-1)^{q} \psi^{(q)}$ is nonincreasing on $[0, \infty)$ for $q \in\{1,2\}$ and $\ell(\boldsymbol{x}) \geq x_{1} \vee \cdots \vee x_{d}$ for any $\boldsymbol{x} \in \mathbb{R}_{+}^{d}$, one has that for any $i \neq j$ and any $\boldsymbol{x} \in\left\{\phi(\boldsymbol{u}), \boldsymbol{u} \in V_{d, i} \cap V_{d, j}\right\}$ and $\boldsymbol{x} \in\left\{\phi(\boldsymbol{u}), \boldsymbol{u} \in V_{d, i}\right\}$,

$$
\begin{aligned}
\left|\ddot{C}_{i j}\{\psi(\boldsymbol{x})\}\right| & \leq \tilde{T}_{1}\left(x_{i}, x_{j}\right)+\tilde{T}_{2}\left(x_{i}, x_{j}\right) \\
\text { and } \quad\left|\ddot{C}_{i i}\{\psi(\boldsymbol{x})\}\right| & \leq \tilde{T}_{1}\left(x_{i}, x_{i}\right)+\tilde{T}_{2}\left(x_{i}, x_{i}\right)+\tilde{T}_{3}\left(x_{i}\right),
\end{aligned}
$$

respectively. Note that for $k \in\{1,2\}$, the term $\tilde{T}_{k}$ is symmetric. To show the inequality (9.1) it thus suffices to prove that for $k \in\{1,2\}$, the function given for all $z_{1}, z_{2} \in\left(0, x_{\psi}\right)$ by $\psi\left(z_{1}\right)\left\{1-\psi\left(z_{1}\right)\right\} \tilde{T}_{k}\left(z_{1}, z_{2}\right)$ is bounded on $\left(0, x_{\psi}\right)^{2}$, and further that the function given for all $z_{1} \in\left(0, x_{\psi}\right)$ by $\psi\left(z_{1}\right)\left\{1-\psi\left(z_{1}\right)\right\} \tilde{T}_{3}\left(z_{1}\right)$ is bounded on $\left(0, x_{\psi}\right)$. First observe that because $-\psi^{\prime}$ is nonincreasing,

$$
\begin{aligned}
\psi\left(z_{1}\right)\left\{1-\psi\left(z_{1}\right)\right\} \tilde{T}_{2}\left(z_{1}, z_{2}\right) & \leq \frac{M \psi\left(z_{1}\right)\left\{1-\psi\left(z_{1}\right)\right\}}{-z_{1} \psi^{\prime}\left(z_{1}\right)}, \\
\psi\left(z_{1}\right)\left\{1-\psi\left(z_{1}\right)\right\} \tilde{T}_{3}\left(z_{1}\right) & \leq \frac{\psi^{\prime \prime}\left(z_{1}\right) \psi\left(z_{1}\right)\left\{1-\psi\left(z_{1}\right)\right\}}{\left\{\psi^{\prime}\left(z_{1}\right)\right\}^{2}} .
\end{aligned}
$$


The function on the right-hand side in the first and second inequality is bounded on $\left(0, x_{\psi}\right)$ by Lemma 9.2 and Lemma 9.3 , respectively.

It remains to consider the function $\tilde{T}_{1}$. For all $z_{1}, z_{2} \in\left(0, x_{\psi}\right)$, denote $h\left(z_{1}, z_{2}\right)=\psi\left(z_{1}\right)\left\{1-\psi\left(z_{1}\right)\right\} \tilde{T}_{1}\left(z_{1}, z_{2}\right)$. First note that because $-\psi^{\prime}$ is decreasing on $\left(0, x_{\psi}\right)$,

$$
h\left(z_{1}, z_{2}\right) \leq \frac{\psi\left(z_{1}\right)\left\{1-\psi\left(z_{1}\right)\right\}}{-z_{1} \psi^{\prime}\left(z_{1}\right)} \frac{\left(z_{1} \vee z_{2}\right) \psi^{\prime \prime}\left(z_{1} \vee z_{2}\right)}{-\psi^{\prime}\left(z_{1} \vee z_{2}\right)}=f\left(z_{1}\right) g\left(z_{1} \vee z_{2}\right),
$$

in terms of $f(x)=\psi(x)\{1-\psi(x)\} /\left\{-x \psi^{\prime}(x)\right\}$ and $g(x)=x \psi^{\prime \prime}(x) /\left\{-\psi^{\prime}(x)\right\}$. Now $f$ is bounded on $\left(0, x_{\psi}\right)$ by Lemma 9.2. Furthermore, $g$ is continuous and because Condition 4.1 holds, it satisfies

$$
\lim _{x \rightarrow 0} g(x)=\lim _{x \rightarrow \infty} \frac{(1 / x)^{2} \psi^{\prime \prime}(1 / x)}{(1 / x)\left\{-\psi^{\prime}(1 / x)\right\}}=1-1 / m
$$

where the last equality follows from Equation (12) in [33]. Therefore, $h$ is bounded on $(0, \kappa]^{2}$ for any $\kappa<x_{\psi}$. To conclude that $h$ is bounded on the entire set $\left(0, x_{\psi}\right)^{2}$, two cases have to be distinguished. First, assume that Condition 4.2 (a) holds. In this case, $x_{\psi}=\infty$ and Equation (7) in [33] implies $\lim _{x \rightarrow \infty} g(x)=$ $s+1$ and hence the upper bound in (9.5) is bounded on $\left(0, x_{\psi}\right)^{2}$. Next, assume that Condition 4.2 (b) holds, and that $-\log (\psi)$ is concave. In this case, the upper bound in (9.5) is too crude because $g(x) \rightarrow \infty$ as $x \rightarrow x_{\psi}$. Instead observe that, because $\psi$ is decreasing,

$$
\begin{aligned}
h\left(z_{1}, z_{2}\right)=\frac{\psi\left(z_{1} \vee z_{2}\right) \psi^{\prime \prime}\left(z_{1} \vee z_{2}\right)}{\left\{\psi^{\prime}\left(z_{1} \vee z_{2}\right)\right\}^{2}} & \frac{\psi\left(z_{1}\right)}{\psi\left(z_{1} \vee z_{2}\right)} \frac{\psi^{\prime}\left(z_{1} \vee z_{2}\right)}{\psi^{\prime}\left(z_{1} \wedge z_{2}\right)} \\
& \leq \frac{\psi\left(z_{1} \vee z_{2}\right) \psi^{\prime \prime}\left(z_{1} \vee z_{2}\right)}{\left\{\psi^{\prime}\left(z_{1} \vee z_{2}\right)\right\}^{2}} \frac{a_{1}^{*}\left(z_{1} \wedge z_{2}\right)}{a_{1}^{*}\left(z_{1} \vee z_{2}\right)},
\end{aligned}
$$

where for any $x \in\left(0, x_{\psi}\right), a_{1}^{*}(x)=\psi(x) /\left\{-\psi^{\prime}(x)\right\}$. From the proof of Lemma 9.3, $\psi(x) \psi^{\prime \prime}(x) /\left\{\psi^{\prime}(x)\right\}^{2} \rightarrow 1$ as $x \rightarrow x_{\psi}$. Furthermore, because $-\log (\psi)$ is concave, $a_{1}^{*}$ is increasing and hence the upper bound in (9.6) is bounded on $\left(0, x_{\psi}\right)^{2} \backslash(0, \kappa]^{2}$ for any $\kappa \in\left(0, x_{\psi}\right)$. Put together, $h$ is bounded on $\left(0, x_{\psi}\right)^{2}$.

\section{Proofs from Section 4}

\subsection{Proofs of Lemmas 4.1 and 4.2}

Proof of Lemma 4.1. For part (i), note that Condition 4.2 (a) with $s>1$ is equivalent to $Z \in \mathcal{M}\left(\Phi_{s}\right)$ with $s>1$. Similarly, Condition 4.2 (b) is equivalent to $Z \in \mathcal{M}(\Lambda)$, and Condition 4.2 (c) implies that $Z$ is bounded from above. In either case, $\mathrm{E}(Z)<\infty$, see, e.g., Chapter 3 in [14]. Before showing that $\hat{\mu} \rightarrow \mathrm{E}(Z)$ as $n \rightarrow \infty$, note that for any positive random variable with finite expectation and a differentiable survival function $\bar{F}$, integrating by parts and a change of variable yields

$$
\int_{0}^{\infty} \bar{F}(t) \mathrm{d} t=\int_{0}^{1}(\bar{F})^{-1}(s) \mathrm{d} s
$$


given that $\lim _{t \rightarrow \infty} t \bar{F}(t)=\lim _{t \rightarrow 0} t \bar{F}(t)=0$. Eq. (10.1) then gives

$$
\int_{0}^{1} \phi(s) \mathrm{d} s=\int_{0}^{\infty} \psi(t) \mathrm{d} t=\mathrm{E}(Z)<\infty,
$$

and hence $\hat{\mu} \rightarrow \mathrm{E}(Z)$ as $n \rightarrow \infty$, as claimed.

To show part (ii), write

$\mathrm{E}(\log Z)=\mathrm{E}\{\log (Z \vee 1)\}+\mathrm{E}\{\log (Z \wedge 1)\}=\mathrm{E}\{\log (Z \vee 1)\}-\mathrm{E}\{\log (1 / Z \vee 1)\}$.

When Condition 4.2 holds, $Z$ is in the domain of attraction of either the Fréchet, the Gumbel or the Weibull distributions. In either case, $\mathrm{E}\{\log (Z \vee 1)\}<\infty$; see Corollary 3.3.32 and Examples 3.3.33 and 3.3.34 in [14]. Furthermore, given that $1-\psi(1 / x)$ is the survival function of $1 / Z$, Condition 4.1 implies that $1 / Z \in \mathcal{M}\left(\Phi_{1 / m}\right)$ and hence $\mathrm{E}\{\log (1 / Z \vee 1)\}<\infty$ again using Example 3.3.33 in [14]. As in part (i), $\hat{\nu} \rightarrow \mathrm{E}(\log Z)$ as $n \rightarrow \infty$ then follows directly from

$$
\mathrm{E}(\log Z)=\int_{0}^{\infty} \psi\{\exp (t)\} \mathrm{d} t=\int_{0}^{1} \log \{\phi(s)\} \mathrm{d} s<\infty,
$$

which holds by Eq. (10.1) given that $\psi\left(e^{t}\right)$ is the survival function of $\log Z$.

Proof of Lemma 4.2. Using the fact that $\log (t)=\int_{0}^{\infty}\{\mathbb{1}(x \leq t)-\mathbb{1}(x \leq 1)\} x^{-1} d x$, for $\boldsymbol{w} \in \Delta_{d}$, write

$$
\begin{aligned}
\mathbb{B}_{n}^{C F G}(\boldsymbol{w})=-\sqrt{n}\left\{-E \log Z+\frac{1}{n} \sum_{i=1}^{n} \log \hat{\xi}_{i}(\boldsymbol{w})+E \log Z-E \log \xi(\boldsymbol{w})\right\} \\
=-\sqrt{n}\left(\frac{1}{n} \sum_{i=1}^{n} \int_{0}^{\infty}\left[\mathbb{1}\left\{x \leq \hat{\xi}_{i}(\boldsymbol{w})\right\}-\mathbb{1}\{x \leq 1\}\right] \frac{d x}{x}\right. \\
\left.\quad-E \int_{0}^{\infty}[\mathbb{1}\{x \leq \xi(\boldsymbol{w})\}-\mathbb{1}\{x \leq 1\}] \frac{d x}{x}\right) \\
=-\sqrt{n}\left(\int_{0}^{\infty}\left[\frac{1}{n} \sum_{i=1}^{n} \mathbb{1}\left\{x \leq \hat{\xi}_{i}(\boldsymbol{w})\right\}-\mathbb{1}\{x \leq 1\}\right] \frac{d x}{x}\right. \\
\left.\quad-\int_{0}^{\infty}[\mathbb{P}\{x \leq \xi(\boldsymbol{w})\}-\mathbb{1}\{x \leq 1\}] \frac{d x}{x}\right) \\
=-\sqrt{n} \int_{0}^{\infty}\left[\frac{1}{n} \sum_{i=1}^{n} \mathbb{1}\left\{\hat{U}_{i 1} \leq \psi\left(w_{1} x\right), \ldots, \hat{U}_{i d} \leq \psi\left(w_{d} x\right)\right\}\right. \\
\left.\quad-\mathbb{P}\left\{U_{i 1} \leq \psi\left(w_{1} x\right), \ldots, U_{i d} \leq \psi\left(w_{d} x\right)\right\}\right] \frac{d x}{x} \\
=-\int_{0}^{\infty} \sqrt{n}\left[\hat{C}_{n}\{\psi(\boldsymbol{w} x)\}-C\{\psi(\boldsymbol{w} x)\}\right] \frac{d x}{x} \\
=\int_{0}^{1} \hat{\mathbb{C}}_{n}[\psi\{-\boldsymbol{w} \log (u)\}] \frac{d u}{u \log u} .
\end{aligned}
$$


Similarly, for the Pickands-type estimator, for $\boldsymbol{w} \in \Delta_{d}$,

$$
\begin{aligned}
\mathbb{B}_{n}^{\mathrm{P}}(\boldsymbol{w}) & =\sqrt{n}\left\{\frac{\sum_{i=1}^{n} \hat{\xi}_{i}(\boldsymbol{w})}{n E(Z)}-\frac{A(\boldsymbol{w})}{E(Z)}\right\} \\
& =\frac{\sqrt{n}}{E(Z)} \int_{0}^{\infty}\left[\frac{1}{n} \sum_{i=1}^{n} \mathbb{1}\left\{\hat{\xi}_{i}(\boldsymbol{w}) \geq x\right\} d x-E\{\xi(\boldsymbol{w})\}\right] d x \\
& =\frac{\sqrt{n}}{E(Z)} \int_{0}^{\infty}\left[\frac{1}{n} \sum_{i=1}^{n} \mathbb{1}\left\{\hat{\xi}_{i}(\boldsymbol{w}) \geq x\right\}-\mathbb{P}\{\xi(\boldsymbol{w})>x\}\right] d x \\
& =\frac{1}{E(Z)} \int_{0}^{\infty} \sqrt{n}\left[\hat{C}_{n}\{\psi(\boldsymbol{w} x)\}-C\{\psi(\boldsymbol{w} x)\}\right] d x \\
& =\frac{1}{E(Z)} \int_{0}^{1} \hat{\mathbb{C}}_{n}[\psi\{-\boldsymbol{w} \log (u)\}] \frac{d u}{u}
\end{aligned}
$$

\subsection{Auxiliary results}

In the following, lemmas that are used in the proof of Proposition 4.2 are stated and proved.

Lemma 10.1. Suppose that Condition 4.1 holds. Then for any $K \in \mathbb{N}$ and $c<1 / K^{1 / m}$, there exists $N_{K} \in \mathbb{N}$ so that for all $n \geq N_{K}$,

$$
\psi\left\{K \phi\left(1-\frac{c}{n}\right)\right\}>\frac{n}{n+1} .
$$

Proof. First note that because $\phi(1-1 / x) \in \mathcal{R}_{-m}$,

$$
\lim _{n \rightarrow \infty} \frac{\phi(1-c / n)}{\phi\{1-1 /(n+1)\}}=c^{m} .
$$

Consequently, because $\phi\{1-1 /(n+1)\} \rightarrow 0$ as $n \rightarrow \infty$,

$$
\begin{gathered}
\lim _{n \rightarrow \infty} \frac{1-\psi\{K \phi(1-c / n)\}}{1-\psi[\phi\{1-1 /(n+1)\}]} \\
=\lim _{n \rightarrow \infty} \frac{1-\psi[K \phi\{1-1 /(n+1)\} \phi(1-c / n) / \phi\{1-1 /(n+1)\}]}{1-\psi[\phi\{1-1 /(n+1)\}]}=c K^{1 / m}
\end{gathered}
$$

Because $c K^{1 / m}<1$ by assumption, the result follows.

Lemma 10.2. (i) If Condition 4.2 holds, then for any $\omega \in(0,1 / 2)$ and $a \in$ $\left(0, x_{\psi}\right), \int_{a}^{x_{\psi}}\{\psi(x)\}^{\omega} / x d x$ is finite.

(ii) If Condition 4.2 (a) holds with $s>2$, then for any $\omega \in(1 / s, 1 / 2)$ and any $a>0, \int_{a}^{\infty}\{\psi(x)\}^{\omega} d x$ is finite. 
(iii) If Condition 2 (b) or (c) holds, then for any $\omega \in(0,1 / 2)$ and any $a \in$ $\left(0, x_{\psi}\right), \int_{a}^{x_{\psi}}\{\psi(x)\}^{\omega} d x$ is finite.

Proof. (i) If Condition 4.2 (a) holds, $x_{\psi}=\infty$ and the integrand has index of regular variation $-s \omega-1<-1$; the integral is thus finite by Karamata's Theorem [14, Theorem A3.6]. If Condition 4.2 (b) holds and $x_{\psi}=\infty$, then $\psi$ is rapidly varying and the result follows from Theorem A3.12 (a) in [14]. If Condition 4.2 (b) holds and $x_{\psi}<\infty$ or Condition (c) is satisfied, then $x_{\psi}<\infty$ and the integrand is bounded on $\left[a, x_{\psi}\right]$.

(ii) Given that the integrand is regularly varying with index $-s \omega<-1$, the result follows from Karamata's Theorem, as in (i).

(iii) In this case, the result follows from Theorem A3.12 (a) in [14] if Condition 4.2 (b) holds and $x_{\psi}=\infty$, and from fact that $x_{\psi}<\infty$ otherwise.

Lemma 10.3. (i) If Condition 4.2 holds, then for any $c \in(0,1)$,

$$
\lim _{n \rightarrow \infty} \sqrt{n} \int_{\phi(c / n)}^{x_{\psi}} \frac{\psi(x)}{x} d x=0 .
$$

(ii) If either Condition 4.2 (a) with $s>2$, (b) or (c) holds, then for any $c \in$ $(0,1)$,

$$
\lim _{n \rightarrow \infty} \sqrt{n} \int_{\phi(c / n)}^{x_{\psi}} \psi(x) d x=0 .
$$

Proof. (i) If Condition 4.2 (a) holds, $x_{\psi}=\infty$. By Karamata's Theorem the integral is a regularly varying function of $\phi(c / n)$ with index $-s$. For some slowly varying function $L$,

$$
\sqrt{n} \int_{\phi(c / n)}^{\infty} \frac{\psi(x)}{x} d x=\sqrt{n}\{\phi(c / n)\}^{-s} L\{\phi(c / n)\} .
$$

Due to the regular variation of $\phi$ at zero, there exists a slowly varying function $L^{*}$ such that

$$
\begin{aligned}
\sqrt{n}\{\phi(c / n)\}^{-s} L\{\phi(c / n)\} & =\sqrt{n}\left\{(n / c)^{1 / s} L^{*}(n / c)\right\}^{-s} L\{\phi(c / n)\} \\
& =\frac{c}{\sqrt{n}} L^{\dagger}(n),
\end{aligned}
$$

where $L^{\dagger}(n)=L^{*}(n / c)^{-s} L\{\phi(c / n)\}$ is a slowly varying function of $n$ Resnick [42, Proposition 0.8 (iv)]. Consequently, the right-hand side of (10.2) converges to zero as $n \rightarrow \infty$.

If Condition 4.2 (b) holds and $x_{\psi}=\infty$, Theorem A3.12 (b) in [14] implies that

$$
\lim _{n \rightarrow \infty} \frac{n}{c} \int_{\phi(c / n)}^{\infty} \frac{\psi(x)}{x} d x=0
$$

from which the result follows at once. Finally, if Condition 4.2 (b) holds and $x_{\psi}<\infty$ or if Condition 4.2 (c) is satisfied, $x_{\psi}<\infty$ and $\psi(x)=0$ for all $x \geq x_{\psi}$. 
Because $\psi$ is decreasing,

$$
\sqrt{n} \int_{\phi(c / n)}^{x_{\psi}} \frac{\psi(x)}{x} d x \leq \sqrt{n} \int_{\phi(c / n)}^{x_{\psi}} \frac{\psi\{\phi(c / n)\}}{x} d x=\frac{\log x_{\psi}-\log \{\phi(c / n)\}}{\sqrt{n} / c} .
$$

Clearly, the last expression converges to zero as $n \rightarrow \infty$.

(ii) If Condition 4.2 (a) holds with $s>2, x_{\psi}=\infty$ and one can argue as in the proof of (i) using Karamata's Theorem that

$$
\sqrt{n} \int_{b_{n}}^{\infty} \psi(x) d x=n^{1 / 2+1 / s-1} L^{\dagger \dagger}(n),
$$

where $L^{\dagger \dagger}$ is slowly varying. Since $1 / 2+1 / s-1<0$, the right-hand side converges to 0 as $n \rightarrow \infty$. If Condition 4.2 (b) holds and $x_{\psi}=\infty$, Theorem A3.12 (b) in [14] and the fact that $\phi(1 / x)$ is slowly varying [7, Theorem 2.4.7] imply that

$$
\lim _{n \rightarrow \infty} \sqrt{n} \int_{\phi(c / n)}^{\infty} \psi(x) d x=\lim _{n \rightarrow \infty} \frac{c \phi(c / n)}{\sqrt{n}} \frac{\int_{\phi(c / n)}^{\infty} \psi(t) d t}{(c / n) \phi(c / n)}=0 .
$$

If Condition 4.2 (b) holds and $x_{\psi}<\infty$ or Condition 4.2 (c) is satisfied, then $x_{\psi}<\infty$. Consequently,

$$
\sqrt{n} \int_{\phi(c / n)}^{x_{\psi}} \psi(x) d x \leq \sqrt{n}(c / n)\left\{x_{\psi}-\phi(c / n)\right\} ;
$$

the last expression clearly converges to zero as $n \rightarrow \infty$.

Remark 10.1. It emerges from the proofs of Lemma 10.2 and 10.3 that these results remain valid if instead of Condition $4.2(b)$ or (c), $\psi$ satisfies the weaker condition that either $x_{\psi}<\infty$, or that $x_{\psi}=\infty$ and $\psi$ is rapidly varying as defined, e.g., on p. 83 in [7].

\subsection{Proof of Proposition 4.2 (a)}

Let $\mathcal{K}$ be a compact subset of $\check{\Delta}_{d}$. For an arbitrary $\boldsymbol{w} \in \Delta_{d}$, set $w_{(1)}=$ $\min _{i=1, \ldots, d} w_{i}$ and $w_{(d)}=\max _{i=1, \ldots, d} w_{i}$. Define, for any $k \in \mathbb{N}$, the set $B_{1 / k}=$ $\left\{\boldsymbol{w} \in \Delta_{d}: w_{(1)} \geq 1 / k\right\}$. Since $\mathcal{K}$ is compact, there exists an integer $K>1$ such that $\mathcal{K} \subset B_{1 / K} \subset \stackrel{\circ}{d}_{d}$. Next, pick an arbitrary $c \in\left(0,1 / K^{1 / m}\right)$ with $m$ from Condition 4.1, and define

$$
a_{n}=\phi\left(1-\frac{c}{n}\right), \quad b_{n}=\phi\left(\frac{c}{n}\right) .
$$

By Lemma 10.1 and because $c<1$, there exists $N_{K} \in \mathbb{N}$ so that for any $n \geq N_{K}$,

$$
c<\frac{n}{n+1} \quad \text { and } \quad \psi\left\{K \phi\left(1-\frac{c}{n}\right)\right\}>\frac{n}{n+1} .
$$


Next, for any $i \geq 1$ and $j \in\{1, \ldots, d\}$, let $U_{i j}=F_{j}\left(X_{i j}\right)$ and set $\boldsymbol{U}_{i}=$ $\left(U_{i 1}, \ldots, U_{i d}\right)$. Let $\alpha_{n}$ be the empirical process pertaining to the (unobservable) sequence $\boldsymbol{U}_{1}, \ldots, \boldsymbol{U}_{n}$, that is, for any $\boldsymbol{u} \in[0,1]^{d}$,

$$
\alpha_{n}(\boldsymbol{u})=\sqrt{n}\left\{C_{n}(\boldsymbol{u})-C(\boldsymbol{u})\right\}, \quad C_{n}(\boldsymbol{u})=\frac{1}{n} \sum_{i=1}^{n} \prod_{j=1}^{d} \mathbb{1}\left(U_{i j} \leq u_{j}\right) .
$$

Furthermore, let $\overline{\mathbb{C}}_{n}$ be the process defined at any $\boldsymbol{u} \in[0,1]^{d}$ as follows:

$$
\overline{\mathbb{C}}_{n}(\boldsymbol{u})=\alpha_{n}(\boldsymbol{u})-\sum_{j=1}^{d} \dot{C}_{j}(\boldsymbol{u}) \alpha_{n}\left(\boldsymbol{u}^{(j)}\right) .
$$

Before proceeding, recall that for any $\boldsymbol{x} \in \mathbb{R}_{+}^{d}, \psi(\boldsymbol{x})=\left(\psi\left(x_{1}\right), \ldots, \psi\left(x_{d}\right)\right)$ and note the following lemma.

Lemma 10.4. As $n \rightarrow \infty, \sup _{\boldsymbol{w} \in B_{1 / K}} \int_{0}^{x_{\psi} / w_{(d)}}\left|\overline{\mathbb{C}}_{n}\{\psi(\boldsymbol{w} x)\}-\hat{\mathbb{C}}_{n}\{\psi(\boldsymbol{w} x)\}\right| \frac{d x}{x}$ converges in probability to 0.

Proof of Lemma 10.4. Using triangle inequality and $a_{n}, b_{n}$ as in (10.3), write, for any $\boldsymbol{w} \in B_{1 / K}$,

$$
\int_{0}^{x_{\psi} / w_{(d)}}\left|\overline{\mathbb{C}}_{n}\{\psi(\boldsymbol{w} x)\}-\hat{\mathbb{C}}_{n}\{\psi(\boldsymbol{w} x)\}\right| \frac{d x}{x} \leq I_{1}(\boldsymbol{w})+I_{2}(\boldsymbol{w})+I_{3}(\boldsymbol{w})+I_{4}(\boldsymbol{w})+I_{5}(\boldsymbol{w}),
$$

where

$$
\begin{gathered}
I_{1}(\boldsymbol{w})=\int_{a_{n} / w_{(1)}}^{b_{n} / w_{(d)}}\left|\hat{\mathbb{C}}_{n}\{\psi(\boldsymbol{w} x)\}-\overline{\mathbb{C}}_{n}\{\psi(\boldsymbol{w} x)\}\right| \frac{d x}{x}, \\
I_{2}(\boldsymbol{w})=\int_{0}^{a_{n} / w_{(1)}}\left|\hat{\mathbb{C}}_{n}\{\psi(\boldsymbol{w} x)\}\right| \frac{d x}{x}, \\
I_{3}(\boldsymbol{w})=\int_{b_{n} / w_{(d)}}^{x_{\psi} / w_{(d)}}\left|\hat{\mathbb{C}}_{n}\{\psi(\boldsymbol{w} x)\}\right| \frac{d x}{x}, \quad I_{4}(\boldsymbol{w})=\int_{0}^{a_{n} / w_{(1)}}\left|\overline{\mathbb{C}}_{n}\{\psi(\boldsymbol{w} x)\}\right| \frac{d x}{x}, \\
I_{5}(\boldsymbol{w})=\int_{b_{n} / w_{(d)}}^{x_{\psi} / w_{(d)}}\left|\overline{\mathbb{C}}_{n}\{\psi(\boldsymbol{w} x)\}\right| \frac{d x}{x} .
\end{gathered}
$$

In the sequel, we show that for any $p \in\{1, \ldots, 5\}, \sup _{\boldsymbol{w} \in B_{1 / K}} I_{p}(\boldsymbol{w}) \rightarrow 0$ in probability as $n \rightarrow \infty$.

Treatment of $I_{1}$. Fix an arbitrary $\boldsymbol{w} \in B_{1 / K}$ and introduce, for any $\omega \in(0,1 / 2)$, the weight function $g_{\omega}$ as in [6]. The latter is given at any $\boldsymbol{u} \in[0,1]^{d}$ by

$$
g_{\omega}(\boldsymbol{u})=\min \left[\bigwedge_{i=1}^{d} u_{j}, \bigwedge_{i=1}^{d}\left\{1-\min _{j=1, \ldots, d}\left(u_{1}, \ldots, u_{j-1}, u_{j+1}, \ldots, u_{d}\right)\right\}\right]^{\omega} .
$$


Because $a_{n} / w_{(1)}<x<b_{n} / w_{(d)}$ implies that, for all $j \in\{1, \ldots, d\}, c / n<$ $\psi\left(w_{j} x\right)<1-c / n$, one has

$$
\begin{aligned}
I_{1}(\boldsymbol{w}) & =\int_{a_{n} / w_{(1)}}^{b_{n} / w_{(d)}}\left|\frac{\hat{\mathbb{C}}_{n}\{\psi(\boldsymbol{w} x)\}}{g_{\omega}\{\psi(\boldsymbol{w} x)\}}-\frac{\overline{\mathbb{C}}_{n}\{\psi(\boldsymbol{w} x)\}}{g_{\omega}\{\psi(\boldsymbol{w} x)\}}\right| \frac{g_{\omega}\{\psi(\boldsymbol{w} x)\}}{x} d x \\
& \leq S_{n} \int_{0}^{x_{\psi} / w_{(d)}} \frac{g_{\omega}\{\psi(\boldsymbol{w} x)\}}{x} d x
\end{aligned}
$$

where

$$
S_{n}=\sup _{\boldsymbol{u} \in[c / n, 1-c / n]^{d}}\left|\frac{\hat{\mathbb{C}}_{n}(\boldsymbol{u})}{g_{\omega}(\boldsymbol{u})}-\frac{\overline{\mathbb{C}}_{n}(\boldsymbol{u})}{g_{\omega}(\boldsymbol{u})}\right| .
$$

By Equation (2.1) in Theorem 2.2 in [6], $S_{n}$ converges to 0 in probability as $n \rightarrow \infty$. The conditions of the latter Theorem are indeed fulfilled because of Proposition 4.1. To conclude that $\sup _{\boldsymbol{w} \in B_{1 / K}} I_{1}(\boldsymbol{w}) \rightarrow 0$ in probability as $n \rightarrow \infty$, it thus suffices to show that $\int_{0}^{x_{\psi} / w_{(d)}} \frac{g_{\omega}\{\psi(\boldsymbol{w} x)\}}{x} d x$ is finite. To this end, note that because $\psi$ is decreasing,

$$
g_{\omega}\{\psi(\boldsymbol{w} x)\} \leq\left[\min \left\{\psi\left(x w_{1}\right), \ldots, \psi\left(x w_{d}\right)\right\}\right]^{\omega}=\left\{\psi\left(w_{(d)} x\right)\right\}^{\omega}
$$

and that, since $w_{j} \leq 1$ for all $j \in\{1, \ldots, d\}$,

$$
g_{\omega}\{\psi(\boldsymbol{w} x)\} \leq\left[1-\min \left\{\psi\left(x w_{1}\right), \ldots, \psi\left(x w_{d}\right)\right\}\right]^{\omega}=\left\{1-\psi\left(w_{(d)} x\right)\right\}^{\omega} .
$$

Choosing an arbitrary $a \in\left(0, x_{\psi}\right)$, one then has

$$
\begin{aligned}
\int_{0}^{x_{\psi} / w_{(d)}} \frac{g_{\omega}\{\psi(\boldsymbol{w} x)\}}{x} d x \leq & \int_{0}^{a / w_{(d)}} \frac{\left\{1-\psi\left(w_{(d)} x\right)\right\}^{\omega}}{x} d x \\
& +\int_{a / w_{(d)}}^{x_{\psi} / w_{(d)}} \frac{\left\{\psi\left(w_{(d)} x\right)\right\}^{\omega}}{x} d x=I_{11}+I_{12}<\infty
\end{aligned}
$$

where

$$
I_{11}=\int_{1 / a}^{\infty} \frac{\{1-\psi(1 / x)\}^{\omega}}{x} d x, \quad I_{12}=\int_{a}^{x_{\psi}} \frac{\{\psi(x)\}^{\omega}}{x} d x .
$$

Indeed, under Condition 4.1, $I_{11}$ is finite by Karamata's Theorem, since the integrand has index of regular variation $-m \omega-1$ which is strictly less than -1 . Finally, $I_{12}$ is finite under Condition 4.2 by Lemma 10.2 (i).

Treatment of $I_{2}$. Without loss of generality, suppose that $n \geq N_{K}$ so that (10.4) holds. Fix an arbitrary $\boldsymbol{w} \in B_{1 / K}$ and observe that from the definition of $B_{1 / K}$ one has, for any $x \in\left(0, a_{n} / w_{(1)}\right)$ and $j \in\{1, \ldots, d\}$,

$$
w_{j} x \leq \frac{w_{j}}{w_{(1)}} \phi\left(1-\frac{c}{n}\right) \leq K \phi\left(1-\frac{c}{n}\right) \text {. }
$$

This and (10.4) imply that

$$
\psi\left(w_{j} x\right) \geq \psi\{K \phi(1-c / n)\}>\frac{n}{n+1} .
$$


Consequently, for any $x \in\left(0, a_{n} / w_{(1)}\right), \hat{C}_{n}\{\psi(\boldsymbol{w} x)\}=1$. Using (1.2), one thus has

$$
I_{2}(\boldsymbol{w})=\sqrt{n} \int_{0}^{a_{n} / w_{(1)}}[1-C\{\psi(\boldsymbol{w} x)\}] \frac{d x}{x}=\sqrt{n} \int_{0}^{a_{n} / w_{(1)}} \frac{1-\psi\{\ell(\boldsymbol{w} x)\}}{x} d x .
$$

Because for any $x>0, \ell(\boldsymbol{w} x)=x \ell(\boldsymbol{w}), \ell(\boldsymbol{w}) \leq 1$, and $w_{(1)} \geq 1 / K$ one further has that

$$
I_{2}(\boldsymbol{w}) \leq \sqrt{n} \int_{w_{(1)} / a_{n}}^{\infty} \frac{1-\psi(1 / x)}{x} d x \leq \sqrt{n} \int_{1 /\left(K a_{n}\right)}^{\infty} \frac{1-\psi(1 / x)}{x} d x .
$$

Next, Karamata's Theorem implies that there exists a slowly varying function $L_{1}$ such that

$$
\sqrt{n} \int_{1 /\left(K a_{n}\right)}^{\infty} \frac{1-\psi(1 / x)}{x} d x=\sqrt{n}\left\{K \phi\left(1-\frac{c}{n}\right)\right\}^{\frac{1}{m}} L_{1}\left[\left\{K \phi\left(1-\frac{c}{n}\right)\right\}^{-1}\right] .
$$

Because $\phi(1-1 / x)$ is regularly varying with index $-m$, there exists a slowly varying function $L_{2}$ such that

$$
\begin{aligned}
\sqrt{n}\left\{K \phi\left(1-\frac{c}{n}\right)\right\}^{\frac{1}{m}} & L_{1}\left[\left\{K \phi\left(1-\frac{c}{n}\right)\right\}^{-1}\right] \\
& =\sqrt{n}\left\{K(n / c)^{-m} L_{2}(n / c)\right\}^{\frac{1}{m}} L_{1}\left[\left\{K \phi\left(1-\frac{c}{n}\right)\right\}^{-1}\right] .
\end{aligned}
$$

Thus $I_{2}(\boldsymbol{w}) \leq n^{-1 / 2} L_{3}(n)$, where $L_{3}(x)=c K^{1 / m} L_{2}(x / c)^{1 / m}$ $\times L_{1}\left[\{K \phi(1-c / x)\}^{-1}\right]$. Because $L_{3}$ is slowly varying [42, Proposition 0.8 (iv)], $n^{-1 / 2} L_{3}(n) \rightarrow 0$ as $n \rightarrow \infty$.

Treatment of $I_{3}$. Without loss of generality, suppose that $n \geq N_{K}$ so that (10.4) holds. Fix an arbitrary $\boldsymbol{w} \in B_{1 / K}$ and observe that if $x \geq b_{n} / w_{(d)}$, $\psi\left(x w_{(d)}\right) \leq c / n<1 /(n+1)$ and consequently $\hat{C}_{n}\{\psi(\boldsymbol{w} x)\}=0$. One thus has

$$
\begin{aligned}
I_{3}(\boldsymbol{w}) & =\sqrt{n} \int_{b_{n} / w_{(d)}}^{x_{\psi} / w_{(d)}} C\{\psi(\boldsymbol{w} x)\} \frac{d x}{x} \\
& \leq \sqrt{n} \int_{b_{n} / w_{(d)}}^{x_{\psi} / w_{(d)}} \frac{\psi\left(w_{(d)} x\right)}{x} d x=\sqrt{n} \int_{b_{n}}^{x_{\psi}} \frac{\psi(x)}{x} d x .
\end{aligned}
$$

The last term in the above inequality is independent of $\boldsymbol{w}$ and converges to 0 as $n \rightarrow \infty$ by Lemma 10.3 (i).

Treatment of $I_{4}$. Recall the second weight function $\tilde{g}_{\omega}$ from [6] given, for all $\boldsymbol{u} \in[0,1]^{d}$, by $\tilde{g}_{\omega}(\boldsymbol{u})=g_{\omega}(\boldsymbol{u})+\mathbb{1}\left\{g_{\omega}(\boldsymbol{u})=0\right\}$. Fix an arbitrary $\boldsymbol{w} \in B_{1 / K}$, let

$$
Z_{n}=\sup _{\boldsymbol{u} \in[0,1]^{d}}\left|\frac{\overline{\mathbb{C}}_{n}(\boldsymbol{u})}{\tilde{g}_{\omega}(\boldsymbol{u})}\right|
$$


and observe that

$$
\begin{aligned}
I_{4}(\boldsymbol{w})=\int_{0}^{a_{n} / w_{(1)}}\left|\frac{\overline{\mathbb{C}}_{n}\{\psi(\boldsymbol{w} x)\}}{\tilde{g}_{\omega}\{\psi(\boldsymbol{w} x)\}}\right| \frac{\tilde{g}_{\omega}\{\psi(\boldsymbol{w} x)\}}{x} d x & \leq \int_{0}^{a_{n} / w_{(1)}} Z_{n} \frac{\tilde{g}_{\omega}\{\psi(\boldsymbol{w} x)\}}{x} d x \\
& \leq Z_{n} \int_{0}^{K a_{n}} \frac{\tilde{g}_{\omega}\{\psi(\boldsymbol{w} x)\}}{x} d x .
\end{aligned}
$$

Given that $Z_{n} \rightsquigarrow \sup _{\boldsymbol{u} \in[0,1]^{d}}\left|\mathbb{C}(\boldsymbol{u}) / \tilde{g}_{\omega}(\boldsymbol{u})\right|$ as $n \rightarrow \infty$ by Theorem 2.2 in [6], it suffices to prove that $\int_{0}^{K a_{n}} \frac{\tilde{g}_{\omega}\{\psi(\boldsymbol{w} x)\}}{x} d x$ converges uniformly to 0 as $n \rightarrow \infty$. To this end, note that $g_{\omega}(\boldsymbol{u})=0$ occurs either when at least one component of $\boldsymbol{u}$ is equal to 0 or at least $d-1$ components are equal to 1 . Given that $a_{n} \rightarrow 0$ as $n \rightarrow \infty$, one thus has, for sufficiently large $n$,

$$
\int_{0}^{K a_{n}} \frac{\tilde{g}_{\omega}\{\psi(\boldsymbol{w} x)\}}{x} d x=\int_{0}^{K a_{n}} \frac{g_{\omega}\{\psi(\boldsymbol{w} x)\}}{x} d x .
$$

Using (10.8), the integral on the right-hand side can be bounded above by

$$
\begin{aligned}
\int_{0}^{K a_{n} / w_{(d)}} \frac{g_{\omega}\{\psi(\boldsymbol{w} x)\}}{x} d x & \leq \int_{0}^{K a_{n} / w_{(d)}} \frac{\left\{1-\psi\left(w_{(d)} x\right)\right\}^{\omega}}{x} d x \\
& =\int_{1 /\left(K a_{n}\right)}^{\infty} \frac{\{1-\psi(1 / x)\}^{\omega}}{x} d x
\end{aligned}
$$

The last expression converges to 0 as $n \rightarrow \infty$, given that it is bounded above by $I_{11}$ in (10.9), which is finite, and given that $a_{n} \rightarrow 0$ as $n \rightarrow \infty$.

Treatment of $I_{5}$. Let $\tilde{g}_{\omega}$ be as in the preceding paragraph concerning $I_{4}$. Fix an arbitrary $\boldsymbol{w} \in B_{1 / K}$ and note that, using (10.7) and performing a change of variable,

$$
\begin{aligned}
I_{5}(\boldsymbol{w}) \leq Z_{n} \int_{b_{n} / w_{(d)}}^{x_{\psi} / w_{(d)}} \frac{\tilde{g}_{\omega}\{\psi(\boldsymbol{w} x)\}}{x} d x & =Z_{n} \int_{b_{n} / w_{(d)}}^{x_{\psi} / w_{(d)}} \frac{g_{\omega}\{\psi(\boldsymbol{w} x)\}}{x} d x \\
& \leq Z_{n} \int_{b_{n}}^{x_{\psi}} \frac{\{\psi(x)\}^{\omega}}{x} d x .
\end{aligned}
$$

The claim follows since $\int_{b_{n}}^{x_{\psi}} \frac{\{\psi(x)\}^{\omega}}{x} d x \rightarrow 0$ as $n \rightarrow \infty$ by Lemma 10.2 (i) given that $b_{n} \rightarrow x_{\psi}$ as $n \rightarrow \infty$.

Returning to the proof of Proposition 4.2 (a), fix an arbitrary $\boldsymbol{w} \in B_{1 / K}$ and observe that from Lemma 4.2 and the fact that $\hat{C}_{n}\{\psi(\boldsymbol{w} x)\}=C\{\psi(\boldsymbol{w} x)\}=0$ whenever $x>x_{\psi} / w_{(d)}$,

$$
\mathbb{B}_{n}^{\mathrm{CFG}}(\boldsymbol{w})=-\int_{0}^{x_{\psi} / w_{(d)}} \hat{\mathbb{C}}_{n}\{\psi(\boldsymbol{w} x)\} \frac{d x}{x} .
$$


Now introduce the process $\overline{\mathbb{B}}_{n}^{\mathrm{CFG}}$ given, for any $\boldsymbol{w} \in \Delta_{d}$, by

$$
\overline{\mathbb{B}}_{n}^{\mathrm{CFG}}(\boldsymbol{w})=-\int_{0}^{x_{\psi} / w_{(d)}} \overline{\mathbb{C}}_{n}\{\psi(\boldsymbol{w} x)\} \frac{d x}{x} .
$$

From Lemma 10.4, it follows that $\sup _{\boldsymbol{w} \in B_{1 / K}}\left|\mathbb{B}_{n}^{\mathrm{CFG}}(\boldsymbol{w})-\overline{\mathbb{B}}_{n}^{\mathrm{CFG}}(\boldsymbol{w})\right|$ converges to zero in probability. It thus remains to show that $\overline{\mathbb{B}}_{n}^{\mathrm{CFG}} \rightsquigarrow \mathbb{B}^{\mathrm{CFG}}$ in $\mathcal{C}\left(B_{1 / K}\right)$ as $n \rightarrow \infty$. To do so, consider the map

$$
\begin{aligned}
\Gamma:\left(\ell^{\infty}\left([0,1]^{d}\right),\|\cdot\|_{\tilde{g}_{\omega}}\right) & \longrightarrow\left(\ell^{\infty}\left(B_{1 / K}\right),\|\cdot\|_{\infty}\right) \\
f & \longrightarrow\left\{\boldsymbol{w} \rightarrow-\int_{0}^{x_{\psi} / w_{(d)}} f\{\psi(\boldsymbol{w} x)\} \frac{d x}{x}\right\},
\end{aligned}
$$

where $\|f\|_{\tilde{g}_{\omega}}=\sup _{\boldsymbol{u} \in[0,1]^{d}}\left|f(\boldsymbol{u}) / \tilde{g}_{\omega}(\boldsymbol{u})\right|$. Let $f_{1}, f_{2}$ be arbitrary functions in $\left(\ell^{\infty}\left([0,1]^{d}\right),\|\cdot\|_{\tilde{g}_{\omega}}\right)$. Then

$$
\begin{aligned}
& \sup _{\boldsymbol{w} \in B_{1 / K}}\left|\Gamma\left(f_{1}\right)-\Gamma\left(f_{2}\right)\right| \\
& =\sup _{\boldsymbol{w} \in B_{1 / K}}\left|-\int_{0}^{x_{\psi} / w_{(d)}} \frac{f_{1}\{\psi(\boldsymbol{w} x)\}-f_{2}\{\psi(\boldsymbol{w} x)\}}{\tilde{g}_{\omega}\{\psi(\boldsymbol{w} x)\}} \frac{\tilde{g}_{\omega}\{\psi(\boldsymbol{w} x)\}}{x} d x\right| \\
& \leq \sup _{\boldsymbol{w} \in B_{1 / K}}\left|\int_{0}^{x_{\psi} / w_{(d)}}\left\|f_{1}-f_{2}\right\|_{\tilde{g}_{\omega}} \frac{\tilde{g}_{\omega}\{\psi(\boldsymbol{w} x)\}}{x} d x\right| \\
& \leq\left\|f_{1}-f_{2}\right\|_{\tilde{g}_{\omega}}\left(I_{11}+I_{12}\right),
\end{aligned}
$$

where the last inequality follows from (10.9). The map $\Gamma$ is thus Lipschitz. Theorem 2.2 in $[6]$ and the Continuous Mapping Theorem then imply that $\overline{\mathbb{B}}_{n}^{\mathrm{CFG}}=\Gamma\left(\overline{\mathbb{C}}_{n}\right) \rightsquigarrow \Gamma(\mathbb{C})=\mathbb{B}^{\mathrm{CFG}}$ as $n \rightarrow \infty$ weakly in $\ell^{\infty}\left(B_{1 / K}\right)$. Since $\mathbb{B}^{\mathrm{CFG}}$ has continuous paths on $B_{1 / K}$, the convergence takes place on $\mathcal{C}\left(B_{1 / K}\right)$.

\subsection{Proof of Proposition 4.2 (b)}

The proof of Proposition 4.2 (b) is similar to the proof of part (a) detailed in Section 10.3. For the sake of brevity, only the differences are pointed out.

Let $\mathcal{K}$ be a compact subset of $\Delta_{d}$. Let $B_{1 / K}$ and $c$ be as in Section 10.3 and $a_{n}, b_{n}$ as in (10.3). Furthermore, assume without loss of generality that $n$ is sufficiently large so that (10.4) holds. Finally, recall the weight function $g_{\omega}$ given in (10.5) for some arbitrary fixed $\omega \in(0,1 / 2)$; if Condition 4.2 (a) holds, $\omega \in(0,1 / 2)$ must in addition be such that $s \omega>1$. The following result is the analogue of Lemma 10.4.

Lemma 10.5. As $n \rightarrow \infty, \sup _{\boldsymbol{w} \in B_{1 / K}} \int_{0}^{x_{\psi} / w_{(d)}}\left|\overline{\mathbb{C}}_{n}\{\psi(\boldsymbol{w} x)\}-\hat{\mathbb{C}}_{n}\{\psi(\boldsymbol{w} x)\}\right| d x$ converges in probability to 0.

Proof of Lemma 10.5. Fix an arbitrary $\boldsymbol{w} \in B_{1 / K}$. Then

$$
\int_{0}^{x_{\psi} / w_{(d)}}\left|\overline{\mathbb{C}}_{n}\{\psi(\boldsymbol{w} x)\}-\hat{\mathbb{C}}_{n}\{\psi(\boldsymbol{w} x)\}\right| d x \leq I_{1}(\boldsymbol{w})+I_{2}(\boldsymbol{w})+I_{3}(\boldsymbol{w})+I_{4}(\boldsymbol{w})+I_{5}(\boldsymbol{w}),
$$


where

$$
\begin{gathered}
I_{1}(\boldsymbol{w})=\int_{a_{n} / w_{(1)}}^{b_{n} / w_{(d)}}\left|\hat{\mathbb{C}}_{n}\{\psi(\boldsymbol{w} x)\}-\overline{\mathbb{C}}_{n}\{\psi(\boldsymbol{w} x)\}\right| d x, \\
I_{2}(\boldsymbol{w})=\int_{0}^{a_{n} / w_{(1)}}\left|\hat{\mathbb{C}}_{n}\{\psi(\boldsymbol{w} x)\}\right| d x, \\
I_{3}(\boldsymbol{w})=\int_{b_{n} / w_{(d)}}^{x_{\psi} / w_{(d)}}\left|\hat{\mathbb{C}}_{n}\{\psi(\boldsymbol{w} x)\}\right| d x, \quad I_{4}(\boldsymbol{w})=\int_{0}^{a_{n} / w_{(1)}}\left|\overline{\mathbb{C}}_{n}\{\psi(\boldsymbol{w} x)\}\right| d x, \\
I_{5}(\boldsymbol{w})=\int_{b_{n} / w_{(d)}}^{x_{\psi} / w_{(d)}}\left|\overline{\mathbb{C}}_{n}\{\psi(\boldsymbol{w} x)\}\right| d x .
\end{gathered}
$$

To prove the claim, we show that for any $p \in\{1 \ldots, 5\}$, $\sup _{\boldsymbol{w} \in B_{1 / K}} I_{p}(\boldsymbol{w}) \rightarrow 0$ in probability as $n \rightarrow \infty$.

Treatment of $I_{1}$. Define $S_{n}$ as in (10.6) and observe that

$$
I_{1}(\boldsymbol{w}) \leq S_{n} \int_{a_{n} / w_{(1)}}^{b_{n} / w_{(d)}} g_{\omega}\{\psi(\boldsymbol{w} x)\} d x \leq S_{n} \int_{0}^{x_{\psi} / w_{(d)}} g_{\omega}\{\psi(\boldsymbol{w} x)\} d x .
$$

For an arbitrary $a \in\left(0, x_{\psi}\right)$ one further has, using (10.7) and (10.8) and the fact that $w_{(d)} \geq 1 / d$,

$$
\int_{0}^{x_{\psi} / w_{(d)}} g_{\omega}\{\psi(\boldsymbol{w} x)\} d x \leq d \int_{0}^{a}\{1-\psi(x)\}^{\omega} d x+d \int_{a}^{x_{\psi}} \psi(x)^{\omega} d x .
$$

The upper bound in the preceding display is finite; this follows from Lemma 10.2 (ii)-(iii) and the fact that $\{1-\psi(x)\}^{\omega}$ is bounded on $[0, a]$. Given that $S_{n}$ converges to 0 in probability as $n \rightarrow \infty$ by Theorem 2.2 in [6], $\sup _{\boldsymbol{w} \in B_{1 / K}} I_{1}(\boldsymbol{w}) \rightarrow 0$ in probability as $n \rightarrow \infty$, as claimed.

Treatment of $I_{2}$. Fix an arbitrary $\boldsymbol{w} \in B_{1 / K}$. Using the same arguments as in the paragraph concerning the treatment of $I_{2}$ in the proof of Lemma 10.4, one has that

$$
I_{2}(\boldsymbol{w}) \leq \sqrt{n} \int_{0}^{a_{n} / w_{(1)}}\{1-\psi(x)\} d x \leq \frac{\sqrt{n}}{w_{(1)}} \phi(1-c / n) \leq K \sqrt{n} \phi(1-c / n) .
$$

Given that $\sqrt{x} \phi(1-c / x)$ is regularly varying of index $1 / 2-m<0$, the expression on the right-hand side converges to 0 as $n \rightarrow \infty$.

Treatment of $I_{3}$. Fix an arbitrary $\boldsymbol{w} \in B_{1 / K}$. Using the same arguments as in the paragraph concerning the treatment of $I_{3}$ in the proof of Lemma 10.4 and the fact that $w_{(d)} \geq 1 / d$, one has that $I_{3}(\boldsymbol{w}) \leq d \sqrt{n} \int_{b_{n}}^{x_{\psi}} \psi(x) d x$. The upper bound converges to 0 as $n \rightarrow \infty$ by Lemma 10.3 (ii).

Treatment of $I_{4}$. Fix an arbitrary $\boldsymbol{w} \in B_{1 / K}$. Arguing as in the paragraph concerning the treatment of $I_{4}$ in the proof of Lemma 10.4 and using the fact 
that $w_{(d)} \geq 1 / d$ one has that

$$
I_{4}(\boldsymbol{w}) \leq Z_{n} \int_{0}^{K a_{n}} g_{\omega}\{\psi(\boldsymbol{w} x)\} d x \leq Z_{n} d \int_{0}^{K a_{n}}\{1-\psi(x)\} d x
$$

The upper bound converges in probability to 0 as $n \rightarrow \infty$, given that $Z_{n}$ converges in distribution by Theorem 2.2 in [6], and $\int_{0}^{K a_{n}}\{1-\psi(x)\} d x \rightarrow 0$ as $n \rightarrow \infty$, given that $a_{n} \rightarrow 0$ as $n \rightarrow \infty$.

Treatment of $I_{5}$. Fix an arbitrary $\boldsymbol{w} \in B_{1 / K}$. Arguing as in the paragraph concerning the treatment of $I_{5}$ in the proof of Lemma 10.4, one has that

$$
I_{5}(\boldsymbol{w}) \leq Z_{n} d \int_{b_{n}}^{x_{\psi}} \psi(x) d x .
$$

As in the preceding paragraph, the claim follows from the fact that $\int_{b_{n}}^{x_{\psi}} \psi(x) d x \rightarrow 0$ as $n \rightarrow \infty$ given that $b_{n} \rightarrow x_{\psi}$ as $n \rightarrow \infty$.

Returning to the proof of Proposition 4.2 (b), introduce the process $\overline{\mathbb{B}}_{n}^{\mathrm{P}}$ given, for all $\boldsymbol{w} \in \Delta_{d}$, by

$$
\overline{\mathbb{B}}_{n}^{\mathrm{P}}(\boldsymbol{w})=\frac{1}{E(Z)} \int_{0}^{x_{\psi} / w_{(d)}} \overline{\mathbb{C}}_{n}\{\psi(\boldsymbol{w} x)\} d x .
$$

From Lemma 4.2 one has that

$$
\mathbb{B}_{n}^{\mathrm{P}}(\boldsymbol{w})=\frac{1}{E(Z)} \int_{0}^{x_{\psi} / x_{(d)}} \hat{\mathbb{C}}_{n}\{\psi(\boldsymbol{w} x)\} d x,
$$

and Lemma 10.5 implies that $\sup _{\boldsymbol{w} \in B_{1 / K}}\left|\mathbb{B}_{n}^{\mathrm{P}}(\boldsymbol{w})-\overline{\mathbb{B}}_{n}^{\mathrm{P}}(\boldsymbol{w})\right| \rightarrow 0$ in probability as $n \rightarrow \infty$. As in the proof of Proposition $4.2(\mathrm{~b})$, one can establish that $\overline{\mathbb{B}}_{n}^{\mathrm{P}} \rightsquigarrow \mathbb{B}^{\mathrm{P}}$ as $n \rightarrow \infty$ in $\mathcal{C}\left(B_{1 / K}\right)$ using Theorem 2.2 in [6] and the Continuous Mapping Theorem featuring the map

$$
\begin{aligned}
\Gamma:\left(\ell^{\infty}\left([0,1]^{d}\right),\|\cdot\|_{\tilde{g}_{\omega}}\right) & \longrightarrow\left(\ell^{\infty}\left(B_{1 / K}\right),\|\cdot\|_{\infty}\right) \\
f & \longrightarrow\left\{\boldsymbol{w} \rightarrow \int_{0}^{x_{\psi} / w_{(d)}} f\{\psi(\boldsymbol{w} x)\} d x\right\},
\end{aligned}
$$

which is easily shown to be Lipschitz.

\section{Detailed simulation study results}

This section contains the detailed results of the simulation study from Section 5 in the form of tables containing the means of errors obtained from 1000 Monte Carlo replicates.

Tables 13, 14 and 15 compare results for logistic (LG) and discrete spectral measure-type (DSM) Pickands dependence functions. Following the notation 
from [15], the parameter choices for the latter are provided below. We have $m=$ 10 and $\boldsymbol{w}^{(d)}$ the matrix of weight parameters, where $d$ denotes de dimension.

$$
\begin{aligned}
\boldsymbol{w}^{(2)}= & {\left[\begin{array}{llllllllll}
1.00 & 0.93 & 0.87 & 0.80 & 0.73 & 0.67 & 0.60 & 0.53 & 0.47 & 0.40 \\
0.00 & 0.07 & 0.13 & 0.20 & 0.27 & 0.33 & 0.40 & 0.47 & 0.53 & 0.60
\end{array}\right], } \\
\boldsymbol{w}^{(4)}= & {\left[\begin{array}{llllllllll}
0.67 & 0.00 & 0.33 & 0.33 & 0.00 & 0.33 & 0.33 & 0.00 & 0.00 & 0.00 \\
0.33 & 1.00 & 0.33 & 0.00 & 0.00 & 0.33 & 0.00 & 0.00 & 0.33 & 0.00 \\
0.00 & 0.00 & 0.33 & 0.67 & 1.00 & 0.00 & 0.33 & 0.67 & 0.00 & 0.00 \\
0.00 & 0.00 & 0.00 & 0.00 & 0.00 & 0.33 & 0.33 & 0.33 & 0.67 & 1.00
\end{array}\right], } \\
\boldsymbol{w}^{(10)}= & {\left[\begin{array}{lllllllllll}
0.33 & 0.00 & 0.00 & 0.33 & 0.00 & 0.00 & 0.00 & 0.33 & 0.00 & 0.00 \\
0.33 & 0.00 & 0.00 & 0.00 & 0.00 & 0.33 & 0.00 & 0.00 & 0.00 & 0.00 \\
0.00 & 0.33 & 0.00 & 0.33 & 0.00 & 0.00 & 0.00 & 0.00 & 0.00 & 0.00 \\
0.00 & 0.33 & 0.67 & 0.00 & 0.00 & 0.00 & 0.00 & 0.00 & 0.00 & 0.00 \\
0.33 & 0.00 & 0.00 & 0.00 & 0.33 & 0.33 & 0.00 & 0.33 & 0.33 & 0.00 \\
0.00 & 0.33 & 0.00 & 0.00 & 0.00 & 0.00 & 0.33 & 0.00 & 0.00 & 0.00 \\
0.00 & 0.00 & 0.33 & 0.00 & 0.33 & 0.00 & 0.00 & 0.00 & 0.00 & 0.00 \\
0.00 & 0.00 & 0.00 & 0.33 & 0.33 & 0.00 & 0.33 & 0.00 & 0.33 & 0.00 \\
0.00 & 0.00 & 0.00 & 0.00 & 0.00 & 0.33 & 0.33 & 0.00 & 0.00 & 0.00 \\
& & 0.00 & 0.00 & 0.00 & 0.00 & 0.33 & 0.33 & 1.00
\end{array}\right] }
\end{aligned}
$$




\begin{tabular}{|c|c|c|c|c|c|c|c|c|c|c|c|c|c|}
\hline \multirow[b]{3}{*}{$\psi$} & \multirow{3}{*}{$\begin{array}{l}n \\
\text { error } \\
\tau(\psi) \mid \text { estimator }\end{array}$} & \multicolumn{4}{|c|}{200} & \multicolumn{4}{|c|}{500} & \multicolumn{4}{|c|}{1000} \\
\hline & & \multicolumn{2}{|c|}{$\operatorname{IRAE}(\times 100)$} & \multicolumn{2}{|c|}{ ISE $(\times 10000)$} & \multicolumn{2}{|c|}{$\operatorname{IRAE}(\times 100)$} & \multicolumn{2}{|c|}{ ISE $(\times 10000)$} & \multicolumn{2}{|c|}{$\operatorname{IRAE}(\times 100)$} & \multicolumn{2}{|c|}{$\operatorname{ISE}(\times 10000)$} \\
\hline & & $\mathrm{CFG}$ & $\mathrm{P}$ & $\mathrm{CFG}$ & $\mathrm{P}$ & $\mathrm{CFG}$ & $\mathrm{P}$ & $\overline{\mathrm{CFG}}$ & $\mathrm{P}$ & $\mathrm{CFG}$ & $\mathrm{P}$ & $\mathrm{CFG}$ & $\mathrm{P}$ \\
\hline \multirow[t]{4}{*}{ Clayton } & $1 / 5$ & 1.39 & 2.42 & 1.97 & 6.08 & 0.86 & 1.76 & 0.75 & 3.16 & 0.64 & 1.33 & 0.42 & 1.82 \\
\hline & $2 / 5$ & 1.49 & 8.3 & 2.27 & 90.48 & 0.92 & 7.84 & 0.87 & 83.79 & 0.69 & 7.66 & 0.48 & 79.73 \\
\hline & $3 / 5$ & 1.59 & 28.97 & 2.68 & $26.55 \times 10^{2}$ & 0.98 & 35.08 & 1.02 & $41.92 \times 10^{2}$ & 0.75 & 36.38 & 0.56 & $44.95 \times 10^{2}$ \\
\hline & $4 / 5$ & 2.25 & 620.74 & 5.38 & $1915 \times 10^{6}$ & 1.24 & $86.32 \times 10$ & 1.64 & $66.08 \times 10^{6}$ & 0.87 & $48.34 \times 10$ & 0.77 & $30.40 \times 10^{5}$ \\
\hline \multirow[t]{4}{*}{ Frank } & $1 / 5$ & 1.38 & 1.84 & 1.9 & 3.43 & 0.84 & 1.16 & 0.72 & 1.33 & 0.64 & 0.87 & 0.41 & 0.77 \\
\hline & $2 / 5$ & 1.36 & 2.23 & 1.89 & 5.08 & 0.84 & 1.5 & 0.71 & 2.27 & 0.62 & 1.06 & 0.38 & 1.14 \\
\hline & $3 / 5$ & 1.31 & 2.9 & 1.75 & 8.73 & 0.82 & 1.86 & 0.68 & 3.56 & 0.61 & 1.39 & 0.37 & 1.9 \\
\hline & $4 / 5$ & 1.41 & 4.35 & 2.05 & 20.9 & 0.81 & 2.78 & 0.68 & 8.01 & 0.59 & 2.14 & 0.35 & 4.65 \\
\hline \multirow[t]{4}{*}{ Gumbel } & $1 / 5$ & 1.43 & 1.85 & 2.08 & 3.48 & 0.91 & 1.19 & 0.84 & 1.43 & 0.67 & 0.88 & 0.45 & 0.78 \\
\hline & $2 / 5$ & 1.49 & 2.27 & 2.23 & 5.23 & 0.95 & 1.49 & 0.92 & 2.25 & 0.69 & 1.1 & 0.48 & 1.21 \\
\hline & $3 / 5$ & 1.58 & 3.23 & 2.56 & 11.15 & 0.97 & 2.25 & 0.97 & 5.12 & 0.72 & 1.66 & 0.51 & 2.74 \\
\hline & $4 / 5$ & 1.97 & 7.46 & 4.07 & 69.08 & 1.11 & 5.57 & 1.27 & 36.18 & 0.79 & 4.32 & 0.62 & 20.31 \\
\hline \multirow[t]{4}{*}{ Joe } & $1 / 5$ & 1.46 & 1.79 & 2.17 & 3.27 & 0.92 & 1.14 & 0.87 & 1.33 & 0.68 & 0.84 & 0.46 & 0.71 \\
\hline & $2 / 5$ & 1.54 & 2.04 & 2.4 & 4.2 & 0.98 & 1.32 & 1 & $\begin{array}{l}1.74 \\
\text { S }\end{array}$ & 0.72 & 0.94 & 0.51 & 0.91 \\
\hline & $3 / 5$ & 1.71 & 2.41 & $\begin{array}{l}2.4 \\
3.1\end{array}$ & 5.78 & $\begin{array}{l}1.05 \\
1.05\end{array}$ & 1.56 & 1.17 & 2.44 & 0.77 & 1.12 & 0.59 & 1.31 \\
\hline & $4 / 5$ & 2.37 & 3.38 & 5.81 & 12.03 & 1.28 & 2.21 & 1.7 & 4.99 & 0.91 & 1.56 & 0.83 & 2.53 \\
\hline
\end{tabular}

Table 2: Average Integrated relative absolute error (IRAEx100) and Integrated squared error (ISEx10000) of $A_{n, c}^{\mathrm{CFG}}$ and $A_{n, c}^{\mathrm{P}}$ for 2-dimensional Archimax copula $C_{\psi, A}$ samples of size $n \in\{200,500,1000\}$. The Pickands dependence function $A$ is LG with parameter $\varrho=2$ so that $\tau(A)=1 / 2$, where $\tau(A)=\tau\left(C_{A}\right)$ is Kendall's tau of the bivariate extreme-value copula $C_{A}$. There are four choices for the Archimedean generator $\psi$, Clayton, Frank, Gumbel and Joe, each with four parameter choices so that $\tau(\psi) \in\{1 / 5,2 / 5,3 / 5,4 / 5\}$, where $\tau(\psi)=\tau\left(C_{\psi}\right)$ is Kendall's tau of the bivariate Archimedean copula $C_{\psi}$. There are 1000 Monte Carlo replicates. 


\begin{tabular}{|c|c|c|c|c|c|c|c|c|c|c|c|c|c|}
\hline \multirow[b]{3}{*}{$\psi$} & \multirow{3}{*}{$\begin{array}{l}n \\
\text { error } \\
\tau(\psi) \mid \text { estimator }\end{array}$} & \multicolumn{4}{|c|}{200} & \multicolumn{4}{|c|}{500} & \multicolumn{4}{|c|}{1000} \\
\hline & & \multicolumn{2}{|c|}{$\operatorname{IRAE}(\times 100)$} & \multicolumn{2}{|c|}{$\operatorname{ISE}(\times 10000)$} & \multicolumn{2}{|c|}{ IRAE $(\times 100)$} & \multicolumn{2}{|c|}{$\operatorname{ISE}(\times 10000)$} & \multicolumn{2}{|c|}{$\operatorname{IRAE}(\times 100)$} & \multicolumn{2}{|c|}{$\operatorname{ISE}(\times 10000)$} \\
\hline & & CFG & $\mathrm{P}$ & $\mathrm{CFG}$ & $\mathrm{P}$ & $\mathrm{CFG}$ & $\mathrm{P}$ & $\mathrm{CFG}$ & $\mathrm{P}$ & $\mathrm{CFG}$ & $\mathrm{P}$ & $\mathrm{CFG}$ & $\mathrm{P}$ \\
\hline \multirow[t]{4}{*}{ Clayton } & $1 / 5$ & 1.44 & 2.47 & 2.16 & 6.62 & 0.9 & 1.78 & 0.84 & 3.37 & 0.65 & 1.31 & 0.43 & 1.81 \\
\hline & $2 / 5$ & 1.52 & 7.84 & 2.41 & 83.82 & 0.93 & 7.93 & 0.9 & 83.3 & 0.67 & 7.1 & 0.46 & 63.43 \\
\hline & $3 / 5$ & 1.63 & 34.37 & 2.87 & $47.27 \times 10^{2}$ & 1 & 36.39 & 1.06 & $50.09 \times 10^{2}$ & 0.7 & 33.13 & 0.51 & $35.04 \times 10^{2}$ \\
\hline & $4 / 5$ & 2.3 & $52.42 \times 10$ & 5.49 & $35.15 \times 10^{5}$ & 1.29 & $75.85 \times 10$ & 1.73 & $18.66 \times 10^{6}$ & 0.86 & $47.71 \times 10$ & 0.76 & $26.10 \times 10^{5}$ \\
\hline \multirow[t]{4}{*}{ Frank } & $1 / 5$ & 1.38 & 1.84 & 2 & 3.55 & 0.87 & 1.16 & 0.78 & 1.4 & 0.62 & 0.83 & 0.4 & 0.72 \\
\hline & $2 / 5$ & 1.37 & 2.29 & 1.96 & 5.52 & 0.87 & 1.43 & 0.77 & 2.13 & 0.61 & 1.03 & 0.39 & 1.1 \\
\hline & $3 / 5$ & 1.33 & 2.89 & 1.82 & 9.12 & 0.83 & 1.88 & 0.71 & 3.67 & 0.58 & 1.35 & 0.36 & 1.93 \\
\hline & $4 / 5$ & 1.48 & 4.27 & 2.26 & 19.98 & 0.86 & 2.81 & 0.75 & 8.51 & 0.58 & 2.05 & 0.35 & 4.43 \\
\hline \multirow[t]{4}{*}{ Gumbel } & $1 / 5$ & 1.43 & 1.8 & 2.12 & 3.37 & 0.93 & 1.17 & 0.86 & 1.41 & 0.66 & 0.84 & 0.46 & 0.75 \\
\hline & $2 / 5$ & 1.5 & 2.22 & 2.37 & 5.17 & 0.94 & 1.46 & 0.9 & 2.22 & 0.69 & 1.08 & 0.51 & 1.21 \\
\hline & $3 / 5$ & 1.59 & 3.23 & 2.69 & 11.29 & 0.97 & 2.18 & 0.98 & 5 & 0.72 & 1.62 & 0.55 & 2.74 \\
\hline & $4 / 5$ & 2.01 & 7.46 & 4.31 & 70.59 & 1.13 & 5.28 & 1.3 & 32.79 & 0.78 & 4.47 & 0.64 & 23.05 \\
\hline \multirow[t]{4}{*}{ Joe } & $1 / 5$ & 1.45 & 1.7 & 2.23 & 3.03 & 0.94 & 1.12 & 0.9 & 1.28 & 0.68 & 0.8 & 0.48 & 0.66 \\
\hline & $2 / 5$ & 1.53 & 1.94 & 2.52 & 3.96 & 0.98 & 1.29 & 1.01 & 1.72 & 0.73 & 0.91 & 0.56 & 0.86 \\
\hline & $3 / 5$ & 1.74 & 2.38 & 3.27 & 5.82 & 1.06 & 1.56 & 1.19 & 2.55 & 0.77 & 1.11 & 0.64 & 1.28 \\
\hline & $4 / 5$ & 2.49 & 3.27 & 6.54 & 11.4 & 1.33 & 2.2 & 1.86 & 5.02 & 0.91 & 1.59 & 0.87 & 2.61 \\
\hline
\end{tabular}

Table 3: Average Integrated relative absolute error (IRAEx100) and Integrated squared error (ISEx10000) of $A_{n, c}^{\mathrm{CFG}}$ and $A_{n, c}^{\mathrm{P}}$ for 2-dimensional Archimax copula $C_{\psi, A}$ samples of size $n \in\{200,500,1000\}$. The Pickands dependence function $A$ is NSD with parameters $\boldsymbol{\alpha}=(1,2), \rho=0.59$, so that $\tau(A)=1 / 2$, where $\tau(A)=\tau\left(C_{A}\right)$ is Kendall's tau of the bivariate extreme-value copula $C_{A}$. There are four choices for the Archimedean generator $\psi$, Clayton, Frank, Gumbel and Joe, each with four parameter choices so that $\tau(\psi) \in\{1 / 5,2 / 5,3 / 5,4 / 5\}$, where $\tau(\psi)=\tau\left(C_{\psi}\right)$ is Kendall's tau of the bivariate Archimedean copula $C_{\psi}$. There are 1000 Monte Carlo replicates. 


\begin{tabular}{|c|c|c|c|c|c|c|c|c|c|c|c|c|c|}
\hline \multirow[b]{3}{*}{$\psi$} & \multirow{3}{*}{$\begin{array}{l}n \\
\text { error } \\
\tau(\psi) \mid \text { estimator }\end{array}$} & \multicolumn{4}{|c|}{200} & \multicolumn{4}{|c|}{500} & \multicolumn{4}{|c|}{1000} \\
\hline & & \multicolumn{2}{|c|}{$\operatorname{IRAE}(\times 100)$} & \multicolumn{2}{|c|}{$\operatorname{ISE}(\times 10000)$} & \multicolumn{2}{|c|}{ IRAE $(\times 100)$} & \multicolumn{2}{|c|}{ ISE $(\times 10000)$} & \multicolumn{2}{|c|}{$\operatorname{IRAE}(\times 100)$} & \multicolumn{2}{|c|}{ ISE $(\times 10000)$} \\
\hline & & CFG & $\mathrm{P}$ & CFG & $\mathrm{P}$ & CFG & $\mathrm{P}$ & CFG & $\mathrm{P}$ & CFG & $\mathrm{P}$ & CFG & $\mathrm{P}$ \\
\hline Clayton & $\begin{array}{l}1 / 5 \\
2 / 5 \\
3 / 5 \\
4 / 5\end{array}$ & $\begin{array}{l}2.89 \\
2.91 \\
3.07 \\
4.05\end{array}$ & $\begin{array}{r}4.66 \\
15.47 \\
71.13 \\
13.12 \times 10^{2}\end{array}$ & $\begin{array}{l}4.23 \\
4.22 \\
4.95 \\
8.76\end{array}$ & $\begin{array}{r}10.44 \\
125.2 \\
67.03 \times 10^{2} \\
36.57 \times 10^{6}\end{array}$ & $\begin{array}{r}1.79 \\
1.84 \\
1.97 \\
2.5\end{array}$ & $\begin{array}{r}3.29 \\
14.31 \\
69.62 \\
19.46 \times 10^{2}\end{array}$ & $\begin{array}{r}1.6 \\
1.71 \\
2.01 \\
3.3\end{array}$ & $\begin{array}{r}5.29 \\
106.94 \\
67.85 \times 10^{2} \\
81.52 \times 10^{6}\end{array}$ & $\begin{array}{r}1.29 \\
1.3 \\
1.41 \\
1.6\end{array}$ & $\begin{array}{r}2.4 \\
13.99 \\
74.4 \\
19.42 \times 10^{2}\end{array}$ & $\begin{array}{r}0.82 \\
0.87 \\
1 \\
1.33\end{array}$ & $\begin{array}{r}2.82 \\
98.9 \\
75.85 \times 10^{2} \\
54.47 \times 10^{6}\end{array}$ \\
\hline Frank & $\begin{array}{l}1 / 5 \\
2 / 5 \\
3 / 5 \\
4 / 5\end{array}$ & $\begin{array}{l}2.76 \\
2.75 \\
2.47 \\
2.54\end{array}$ & $\begin{array}{r}3.21 \\
3.85 \\
5.3 \\
8.28\end{array}$ & $\begin{array}{l}3.86 \\
3.82 \\
3.08 \\
3.37\end{array}$ & $\begin{array}{r}5.01 \\
7.23 \\
14.01 \\
36.42\end{array}$ & $\begin{array}{l}1.67 \\
1.64 \\
1.54 \\
1.49\end{array}$ & $\begin{array}{l}1.99 \\
2.45 \\
3.24 \\
4.88\end{array}$ & $\begin{array}{l}1.39 \\
1.37 \\
1.17 \\
1.13\end{array}$ & $\begin{array}{r}1.98 \\
2.98 \\
5.26 \\
11.67\end{array}$ & $\begin{array}{r}1.23 \\
1.18 \\
1.1 \\
1.05\end{array}$ & $\begin{array}{l}1.39 \\
1.72 \\
2.41 \\
3.68\end{array}$ & $\begin{array}{r}0.75 \\
0.68 \\
0.6 \\
0.55\end{array}$ & $\begin{array}{r}0.96 \\
1.49 \\
2.9 \\
6.76\end{array}$ \\
\hline Gumbel & $\begin{array}{l}1 / 5 \\
2 / 5 \\
3 / 5 \\
4 / 5\end{array}$ & $\begin{array}{r}2.96 \\
3.02 \\
3.13 \\
3.7\end{array}$ & $\begin{array}{r}3.17 \\
3.96 \\
5.8 \\
13.8\end{array}$ & $\begin{array}{l}4.35 \\
4.57 \\
5.02 \\
7.29\end{array}$ & $\begin{array}{r}4.96 \\
7.67 \\
16.48 \\
10.46 \times 10\end{array}$ & $\begin{array}{r}1.82 \\
1.9 \\
1.97 \\
2.21\end{array}$ & $\begin{array}{l}2.07 \\
2.66 \\
4.07 \\
9.94\end{array}$ & $\begin{array}{r}1.67 \\
1.8 \\
1.96 \\
2.53\end{array}$ & $\begin{array}{r}2.15 \\
3.49 \\
8.11 \\
50\end{array}$ & $\begin{array}{l}1.32 \\
1.34 \\
1.34 \\
1.45\end{array}$ & $\begin{array}{l}1.43 \\
1.82 \\
2.88 \\
7.84\end{array}$ & $\begin{array}{l}0.86 \\
0.88 \\
0.89 \\
1.06\end{array}$ & $\begin{array}{r}1.02 \\
1.66 \\
4.14 \\
30.24\end{array}$ \\
\hline Joe & $\begin{array}{l}1 / 5 \\
2 / 5 \\
3 / 5 \\
4 / 5\end{array}$ & $\begin{array}{l}3.08 \\
3.23 \\
3.51 \\
4.52\end{array}$ & $\begin{array}{l}3.13 \\
3.56 \\
4.34 \\
6.02\end{array}$ & $\begin{array}{r}4.7 \\
5.31 \\
6.61 \\
11.26\end{array}$ & $\begin{array}{r}4.81 \\
6.28 \\
9.27 \\
17.85\end{array}$ & $\begin{array}{l}1.86 \\
1.99 \\
2.17 \\
2.65\end{array}$ & $\begin{array}{l}1.99 \\
2.28 \\
2.82 \\
3.88\end{array}$ & $\begin{array}{l}1.74 \\
1.96 \\
2.39 \\
3.61\end{array}$ & $\begin{array}{r}1.96 \\
2.6 \\
3.93 \\
7.54\end{array}$ & $\begin{array}{l}1.37 \\
1.43 \\
1.52 \\
1.78\end{array}$ & $\begin{array}{l}1.37 \\
1.61 \\
1.98 \\
2.77\end{array}$ & $\begin{array}{l}0.93 \\
1.04 \\
1.18 \\
1.63\end{array}$ & $\begin{array}{r}0.94 \\
1.3 \\
1.96 \\
3.86\end{array}$ \\
\hline
\end{tabular}

Table 4: Average Integrated relative absolute error (IRAEx100) and Integrated squared error (ISEx10000) of $A_{n, c}^{\mathrm{CFG}}$ and $A_{n, c}^{\mathrm{P}}$ for 4-dimensional Archimax copula $C_{\psi, A}$ samples of size $n \in\{200,500,1000\}$. The Pickands dependence function $A$ is $\mathbf{L G}$ with parameter $\varrho=2$ so that $\tau(A)=1 / 2$, where $\tau(A)=\tau\left(C_{A}\right)$ is the averaged Kendall's tau across all bivariate margins of $C_{A}$. There are four choices for the Archimedean generator $\psi$, Clayton. Frank, Gumbel and Joe, each with four parameter choices so that $\tau(\psi) \in\{1 / 5,2 / 5,3 / 5,4 / 5\}$, where $\tau(\psi)=\tau\left(C_{\psi}\right)$ is Kendall's tau of the bivariate Archimedean copula $C_{\psi}$. There are 1000 Monte Carlo replicates. 


\begin{tabular}{|c|c|c|c|c|c|c|c|c|c|c|c|c|c|}
\hline \multirow[b]{3}{*}{$\psi$} & \multirow{3}{*}{$\begin{array}{l}n \\
\text { error } \\
\tau(\psi) \mid \text { estimator }\end{array}$} & \multicolumn{4}{|c|}{200} & \multicolumn{4}{|c|}{500} & \multicolumn{4}{|c|}{1000} \\
\hline & & \multicolumn{2}{|c|}{$\operatorname{IRAE}(\times 100)$} & \multicolumn{2}{|c|}{ ISE $(\times 10000)$} & \multicolumn{2}{|c|}{ IRAE $(\times 100)$} & \multicolumn{2}{|c|}{$\operatorname{ISE}(\times 10000)$} & \multicolumn{2}{|c|}{$\operatorname{IRAE}(\times 100)$} & \multicolumn{2}{|c|}{$\operatorname{ISE}(\times 10000)$} \\
\hline & & $\mathrm{CFG}$ & $\mathrm{P}$ & $\mathrm{CFG}$ & $\mathrm{P}$ & $\mathrm{CFG}$ & $\mathrm{P}$ & $\mathrm{CFG}$ & $P$ & CFG & $\mathrm{P}$ & $\mathrm{CFG}$ & $\mathrm{P}$ \\
\hline \multirow[t]{3}{*}{ Clayton } & $\begin{array}{l}1 / 5 \\
2 / 5\end{array}$ & $\begin{array}{l}2.08 \\
2.13\end{array}$ & $\begin{array}{r}3.53 \\
12.16\end{array}$ & $\begin{array}{r}1.8 \\
1.91\end{array}$ & $\begin{array}{r}5.11 \\
69.53\end{array}$ & $\begin{array}{l}1.34 \\
1.33\end{array}$ & $\begin{array}{r}2.47 \\
11.54\end{array}$ & $\begin{array}{l}0.75 \\
0.75\end{array}$ & $\begin{array}{r}2.56 \\
63.33\end{array}$ & $\begin{array}{l}0.93 \\
0.94\end{array}$ & $\begin{array}{r}1.91 \\
10.99\end{array}$ & $\begin{array}{l}0.36 \\
0.38\end{array}$ & $\begin{array}{r}1.52 \\
57.14\end{array}$ \\
\hline & $3 / 5$ & $\begin{array}{l}2.10 \\
2.41\end{array}$ & $\begin{array}{l}2.16 \\
60.02\end{array}$ & $\begin{array}{l}1.91 \\
2.57\end{array}$ & $\begin{array}{r}69.53 \\
46.99 \times 10^{2}\end{array}$ & $\begin{array}{l}1.33 \\
1.51\end{array}$ & $\begin{array}{l}11.54 \\
56.85\end{array}$ & $\begin{array}{l}0.75 \\
0.97\end{array}$ & $\begin{array}{r}63.33 \\
45.89 \times 10^{2}\end{array}$ & $\begin{array}{l}0.94 \\
1.06\end{array}$ & $\begin{array}{l}10.99 \\
55.34\end{array}$ & $\begin{array}{l}0.38 \\
0.47\end{array}$ & $\begin{array}{r}5.14 \\
36.54 \times 10^{2}\end{array}$ \\
\hline & $4 / 5$ & 3.55 & $13.92 \times 10^{2}$ & 5.28 & $35.28 \times 10^{6}$ & 1.99 & $12.48 \times 10^{2}$ & 1.67 & $37.26 \times 10^{6}$ & 1.28 & $22.95 \times 10^{2}$ & 0.68 & $25.54 \times 10^{7}$ \\
\hline \multirow[t]{4}{*}{ Frank } & $1 / 5$ & 2.02 & 2.53 & 1.65 & 2.67 & 1.25 & 1.6 & 0.66 & 1.07 & 0.88 & 1.15 & 0.33 & 0.55 \\
\hline & $2 / 5$ & 1.9 & 3.04 & 1.48 & 3.82 & 1.18 & 2.01 & 0.6 & 1.65 & 0.85 & 1.37 & 0.31 & 0.78 \\
\hline & $3 / 5$ & 1.86 & 4.07 & 1.48 & 6.96 & 1.19 & 2.68 & 0.6 & 2.98 & 0.82 & 1.85 & 0.28 & 1.42 \\
\hline & $4 / 5$ & 2.18 & 6.12 & 2.02 & 16.04 & 1.2 & 4.03 & 0.61 & 6.96 & 0.77 & 2.88 & 0.25 & 3.45 \\
\hline \multirow[t]{4}{*}{ Gumbel } & $1 / 5$ & 2.08 & 2.5 & 1.81 & 2.6 & 1.34 & 1.66 & 0.76 & 1.14 & 0.93 & 1.16 & 0.36 & 0.56 \\
\hline & $2 / 5$ & 2.13 & 3.09 & 1.93 & 3.95 & 1.36 & 2.07 & 0.8 & 1.78 & 0.96 & 1.48 & 0.39 & 0.91 \\
\hline & $3 / 5$ & 2.31 & 4.48 & 2.29 & 8.34 & 1.42 & 3.1 & 0.87 & 4.03 & 0.98 & 2.27 & 0.41 & 2.16 \\
\hline & $4 / 5$ & 3.09 & 11.23 & 3.96 & 61.4 & 1.7 & 7.8 & 1.21 & 28.63 & 1.12 & 6.09 & 0.53 & 15.69 \\
\hline \multirow[t]{4}{*}{ Joe } & $1 / 5$ & 2.17 & 2.45 & 1.97 & 2.51 & 1.37 & 1.6 & 0.81 & 1.06 & 0.96 & 1.11 & 0.39 & 0.51 \\
\hline & $2 / 5$ & 2.28 & 2.75 & 2.25 & 3.11 & 1.46 & 1.85 & 0.92 & 1.39 & $\begin{array}{l}1.01 \\
1.01\end{array}$ & $\begin{array}{l}1.27 \\
\end{array}$ & 0.43 & 0.67 \\
\hline & $3 / 5$ & 2.66 & 3.43 & 3.09 & 4.83 & 1.6 & 2.17 & 1.11 & $\begin{array}{l}1.94 \\
\end{array}$ & 1.09 & 1.54 & 0.51 & 0.98 \\
\hline & $4 / 5$ & 3.82 & 4.88 & 6.06 & 10.06 & 2.1 & 3.02 & 1.79 & 3.78 & 1.36 & 2.16 & 0.76 & 1.92 \\
\hline
\end{tabular}

Table 5: Average Integrated relative absolute error (IRAEx100) and Integrated squared error (ISEx10000) of $A_{n, c}^{\mathrm{CFG}}$ and $A_{n, c}^{\mathrm{P}}$ for 4-dimensional Archimax copula $C_{\psi, A}$ samples of size $n \in\{200,500,1000\}$. The Pickands dependence function $A$ is NSD with parameters $\boldsymbol{\alpha}=(1,2,3,4), \rho=0.59, \tau(A)=1 / 2$, where $\tau(A)=\tau\left(C_{A}\right)$ is the averaged Kendall's tau across all bivariate margins of $C_{A}$. There are four choices for the Archimedean generator $\psi$, Clayton, Frank, Gumbel and Joe, each with four parameter choices so that $\tau(\psi) \in\{1 / 5,2 / 5,3 / 5,4 / 5\}$, where $\tau(\psi)=\tau\left(C_{\psi}\right)$ is Kendall's tau of the bivariate Archimedean copula $C_{\psi}$. There are 1000 Monte Carlo replicates. 


\begin{tabular}{|c|c|c|c|c|c|c|c|c|c|c|c|c|c|}
\hline \multirow[b]{3}{*}{$\psi$} & \multirow{3}{*}{$\begin{array}{l}n \\
\text { error } \\
\tau(\psi) \mid \text { estimator } \\
\end{array}$} & \multicolumn{4}{|c|}{200} & \multicolumn{4}{|c|}{500} & \multicolumn{4}{|c|}{1000} \\
\hline & & \multicolumn{2}{|c|}{$\operatorname{IRAE}(\times 100)$} & \multicolumn{2}{|c|}{$\operatorname{ISE}(\times 10000)$} & \multicolumn{2}{|c|}{$\operatorname{IRAE}(\times 100)$} & \multicolumn{2}{|c|}{$\operatorname{ISE}(\times 10000)$} & \multicolumn{2}{|c|}{$\operatorname{IRAE}(\times 100)$} & \multicolumn{2}{|c|}{$\mathrm{ISE}(\times 10000)$} \\
\hline & & CFG & $\mathrm{P}$ & CFG & $\mathrm{P}$ & CFG & $\mathrm{P}$ & CFG & $\mathrm{P}$ & CFG & $\mathrm{P}$ & CFG & $\mathrm{P}$ \\
\hline \multirow[t]{3}{*}{ Clayton } & $\begin{array}{ll}1 / 5 \\
2 / 5\end{array}$ & $\begin{array}{l}4.76 \\
4.98\end{array}$ & $\begin{array}{r}7.23 \\
24.06\end{array}$ & $\begin{array}{l}4.62 \\
5.03\end{array}$ & $\begin{array}{r}10.27 \\
11.74 \times 10\end{array}$ & $\begin{array}{r}3.02 \\
3.1\end{array}$ & $\begin{array}{r}4.98 \\
22.02\end{array}$ & $\begin{array}{r}1.89 \\
2\end{array}$ & $\begin{array}{r}4.93 \\
91.01\end{array}$ & $\begin{array}{l}2.15 \\
2.28\end{array}$ & $\begin{array}{r}3.89 \\
21.08\end{array}$ & $\begin{array}{l}0.94 \\
1.07\end{array}$ & $\begin{array}{r}3 \\
82.74\end{array}$ \\
\hline & $3 / 5$ & $\begin{array}{l}4.90 \\
5.22\end{array}$ & $\begin{array}{r}24.00 \\
11.19 \times 10\end{array}$ & $\begin{array}{l}5.58 \\
5.05\end{array}$ & $55.04 \times 10^{2}$ & $\begin{array}{l}0.1 \\
3.3\end{array}$ & $10.00 \times 10$ & 2.26 & $44.32 \times 10^{2}$ & $\begin{array}{l}2.20 \\
2.38\end{array}$ & $\begin{array}{r}21.00 \\
10.69 \times 10\end{array}$ & 1.17 & $69.58 \times 10^{2}$ \\
\hline & $4 / 5$ & 6.13 & $85.86 \times 10^{2}$ & 8.41 & $31.67 \times 10^{8}$ & 3.75 & $33.04 \times 10^{2}$ & 2.97 & $11.26 \times 10^{7}$ & 2.66 & $59.59 \times 10^{2}$ & 1.47 & $48.02 \times 10^{7}$ \\
\hline \multirow[t]{4}{*}{ Frank } & $1 / 5$ & 4.46 & 4.72 & 4.12 & 4.56 & 2.86 & 2.94 & 1.69 & 1.78 & 2.14 & 2.03 & 0.93 & 0.84 \\
\hline & $2 / 5$ & 4.12 & 5.56 & 3.45 & 6.31 & 2.76 & 3.76 & 1.55 & 2.86 & 1.94 & 2.56 & 0.77 & 1.34 \\
\hline & $3 / 5$ & 3.85 & 8.08 & 3.02 & 13.16 & 2.44 & 5.03 & 1.25 & 5.24 & 1.8 & 3.47 & 0.67 & 2.48 \\
\hline & $4 / 5$ & 3.51 & 12.54 & 2.63 & 32.74 & 2.19 & 7.81 & 1.01 & 12.47 & 1.57 & 5.55 & 0.51 & 6.21 \\
\hline \multirow[t]{4}{*}{ Gumbel } & $1 / 5$ & 4.93 & 4.79 & 5.05 & 4.73 & 3.16 & 3.12 & 2.08 & 2 & 2.18 & 2.14 & 1.01 & 0.94 \\
\hline & $2 / 5$ & 5.12 & 5.86 & 5.5 & 7.01 & 3.24 & 3.89 & 2.19 & 3.1 & 2.21 & 2.69 & 1.04 & 1.5 \\
\hline & $3 / 5$ & 5.32 & 9.01 & 6.03 & 16.06 & 3.27 & 6.07 & 2.24 & 7.32 & 2.27 & 4.48 & 1.08 & 3.97 \\
\hline & $4 / 5$ & 5.9 & 21.96 & 7.85 & $10.24 \times 10$ & 3.49 & 15.54 & 2.57 & 48.2 & 2.38 & 12.91 & 1.21 & 32.38 \\
\hline \multirow[t]{4}{*}{ Joe } & $1 / 5$ & 5.09 & 4.78 & 5.41 & 4.77 & 3.25 & 3.1 & 2.21 & 1.98 & 2.28 & 2.16 & 1.08 & 0.95 \\
\hline & $2 / 5$ & 5.46 & 5.66 & 6.4 & 6.51 & 3.42 & 3.51 & 2.44 & 2.55 & 2.39 & 2.51 & 1.2 & 1.28 \\
\hline & $3 / 5$ & 5.85 & 6.94 & 7.47 & 9.95 & 3.65 & 4.35 & 2.78 & 3.85 & 2.53 & 3.02 & 1.33 & 1.85 \\
\hline & $4 / 5$ & 7.31 & 9.77 & 12 & 20.46 & 4.21 & 6.22 & 3.76 & 7.9 & 2.8 & 4.39 & 1.64 & 3.91 \\
\hline
\end{tabular}

Table 6: Average Integrated relative absolute error (IRAEx100) and Integrated squared error (ISEx10000) of $A_{n, c}^{\mathrm{CFG}}$ and $A_{n, c}^{\mathrm{P}}$ for 10-dimensional Archimax copula $C_{\psi, A}$ samples of size $n \in\{200,500,1000\}$. The Pickands dependence function $A$ is LG with parameter $\varrho=2$ so that $\tau(A)=1 / 2$, where $\tau(A)=\tau\left(C_{A}\right)$ is the averaged Kendall's tau across all bivariate margins of $C_{A}$. There are four choices for the Archimedean generator $\psi$, Clayton, Frank, Gumbel and Joe, each with four parameter choices so that $\tau(\psi) \in\{1 / 5,2 / 5,3 / 5,4 / 5\}$, where $\tau(\psi)=\tau\left(C_{\psi}\right)$ is Kendall's tau of the bivariate Archimedean copula $C_{\psi}$. There are 1000 Monte Carlo replicates. 


\begin{tabular}{|c|c|c|c|c|c|c|c|c|c|c|c|c|c|}
\hline \multirow[b]{3}{*}{$\psi$} & \multirow{3}{*}{$\begin{array}{l}n \\
\text { error } \\
\tau(\psi) \mid \text { estimator }\end{array}$} & \multicolumn{4}{|c|}{200} & \multicolumn{4}{|c|}{500} & \multicolumn{4}{|c|}{1000} \\
\hline & & \multicolumn{2}{|c|}{$\operatorname{IRAE}(\times 100)$} & \multicolumn{2}{|c|}{$\operatorname{ISE}(\times 10000)$} & \multicolumn{2}{|c|}{ IRAE $(\times 100)$} & \multicolumn{2}{|c|}{ ISE $(\times 10000)$} & \multicolumn{2}{|c|}{$\operatorname{IRAE}(\times 100)$} & \multicolumn{2}{|c|}{ ISE $(\times 10000)$} \\
\hline & & CFG & $\mathrm{P}$ & CFG & $\mathrm{P}$ & CFG & $\mathrm{P}$ & CFG & $\mathrm{P}$ & CFG & $\mathrm{P}$ & CFG & $\mathrm{P}$ \\
\hline Clayton & $\begin{array}{l}1 / 5 \\
2 / 5 \\
3 / 5 \\
4 / 5\end{array}$ & $\begin{array}{l}4.65 \\
4.95 \\
5.28 \\
6.25\end{array}$ & $\begin{array}{r}7.27 \\
24.09 \\
106 \\
65.85 \times 10^{2}\end{array}$ & $\begin{array}{l}4.76 \\
5.19 \\
6.13 \\
8.88\end{array}$ & $\begin{array}{r}10.99 \\
122.69 \\
57.55 \times 10^{2} \\
10.76 \times 10^{8}\end{array}$ & $\begin{array}{l}3.05 \\
3.21 \\
3.43 \\
3.89\end{array}$ & $\begin{array}{r}5.23 \\
22.63 \\
112.67 \\
61.49 \times 10^{2}\end{array}$ & $\begin{array}{l}1.99 \\
2.19 \\
2.52 \\
3.42\end{array}$ & $\begin{array}{r}5.59 \\
106.37 \\
73.89 \times 10^{2} \\
42.84 \times 10^{5}\end{array}$ & $\begin{array}{l}2.15 \\
2.18 \\
2.44 \\
2.81\end{array}$ & $\begin{array}{r}3.97 \\
22.14 \\
10.85 \times 10 \\
46.92 \times 10^{2}\end{array}$ & $\begin{array}{l}1.02 \\
1.04 \\
1.33 \\
1.74\end{array}$ & $\begin{array}{r}3.27 \\
98.17 \\
60.78 \times 10^{2} \\
13.06 \times 10^{7}\end{array}$ \\
\hline Frank & $\begin{array}{l}1 / 5 \\
2 / 5 \\
3 / 5 \\
4 / 5\end{array}$ & $\begin{array}{l}4.45 \\
4.24 \\
3.95 \\
3.76\end{array}$ & $\begin{array}{r}4.73 \\
5.67 \\
7.63 \\
12.32\end{array}$ & $\begin{array}{r}4.28 \\
3.81 \\
3.4 \\
3.15\end{array}$ & $\begin{array}{r}4.76 \\
6.82 \\
12.55 \\
34.04\end{array}$ & $\begin{array}{l}2.98 \\
2.77 \\
2.55 \\
2.31\end{array}$ & $\begin{array}{r}3.03 \\
3.73 \\
5.01 \\
7.9\end{array}$ & $\begin{array}{r}1.9 \\
1.68 \\
1.42 \\
1.19\end{array}$ & $\begin{array}{r}1.99 \\
3.01 \\
5.38 \\
13.44\end{array}$ & $\begin{array}{l}2.13 \\
1.99 \\
1.87 \\
1.68\end{array}$ & $\begin{array}{r}2.1 \\
2.52 \\
3.59 \\
5.83\end{array}$ & $\begin{array}{l}0.98 \\
0.86 \\
0.78 \\
0.63\end{array}$ & $\begin{array}{r}0.98 \\
1.39 \\
2.76 \\
7.36\end{array}$ \\
\hline Gumbel & $\begin{array}{l}1 / 5 \\
2 / 5 \\
3 / 5 \\
4 / 5\end{array}$ & $\begin{array}{l}4.77 \\
5.01 \\
5.33 \\
6.18\end{array}$ & $\begin{array}{l}4.76 \\
6.05 \\
9.27 \\
21.9\end{array}$ & $\begin{array}{l}5.01 \\
5.49 \\
6.26 \\
8.57\end{array}$ & $\begin{array}{r}4.94 \\
7.96 \\
17.84 \\
103.21\end{array}$ & $\begin{array}{r}3.09 \\
3.2 \\
3.35 \\
3.65\end{array}$ & $\begin{array}{r}3.04 \\
3.93 \\
6.24 \\
16.16\end{array}$ & $\begin{array}{l}2.06 \\
2.25 \\
2.47 \\
3.01\end{array}$ & $\begin{array}{r}1.99 \\
3.29 \\
8.19 \\
53.95\end{array}$ & $\begin{array}{l}2.33 \\
2.34 \\
2.36 \\
2.45\end{array}$ & $\begin{array}{r}2.22 \\
2.8 \\
4.48 \\
12.99\end{array}$ & $\begin{array}{l}1.18 \\
1.21 \\
1.22 \\
1.36\end{array}$ & $\begin{array}{r}1.08 \\
1.69 \\
4.28 \\
35.38\end{array}$ \\
\hline Joe & $\begin{array}{l}1 / 5 \\
2 / 5 \\
3 / 5 \\
4 / 5\end{array}$ & $\begin{array}{l}4.97 \\
5.33 \\
5.82 \\
7.41\end{array}$ & $\begin{array}{l}4.69 \\
5.46 \\
6.81 \\
9.42\end{array}$ & $\begin{array}{r}5.54 \\
6.49 \\
7.81 \\
12.75\end{array}$ & $\begin{array}{r}4.83 \\
6.42 \\
9.88 \\
19.47\end{array}$ & $\begin{array}{l}3.24 \\
3.42 \\
3.74 \\
4.32\end{array}$ & $\begin{array}{r}3 \\
3.54 \\
4.38 \\
6.03\end{array}$ & $\begin{array}{r}2.29 \\
2.6 \\
3.12 \\
4.26\end{array}$ & $\begin{array}{l}1.98 \\
2.68 \\
4.11 \\
7.93\end{array}$ & $\begin{array}{r}2.47 \\
2.6 \\
2.72 \\
3.08\end{array}$ & $\begin{array}{r}2.2 \\
2.59 \\
3.17 \\
4.36\end{array}$ & $\begin{array}{l}1.34 \\
1.51 \\
1.66 \\
2.12\end{array}$ & $\begin{array}{l}1.08 \\
1.45 \\
2.16 \\
4.18\end{array}$ \\
\hline
\end{tabular}

Table 7: Average Integrated relative absolute error (IRAEx100) and Integrated squared error (ISEx10000) of $A_{n, c}^{\mathrm{CFG}}$ and $A_{n, c}^{\mathrm{P}}$ for 10-dimensional Archimax copula $C_{\psi, A}$ samples of size $n \in\{200,500,1000\}$. The Pickands dependence function $A$ is NSD with parameters $\boldsymbol{\alpha}=(1,1,1,1,2,2,2,3,3,4), \rho=0.69, \tau(A)=1 / 2$, where $\tau(A)=\tau\left(C_{A}\right)$ is the averaged Kendall's tau across all bivariate margins of $C_{A}$. There are four choices for the Archimedean generator $\psi$, Clayton, Frank, Gumbel and Joe, each with four parameter choices so that $\tau(\psi) \in\{1 / 5,2 / 5,3 / 5,4 / 5\}$, where $\tau(\psi)=\tau\left(C_{\psi}\right)$ is Kendall's tau of the bivariate Archimedean copula $C_{\psi}$. There are 1000 Monte Carlo replicates. 


\begin{tabular}{|c|c|c|c|c|c|c|c|c|c|c|c|c|c|c|c|c|c|c|c|c|c|c|c|c|c|}
\hline \multirow[b]{3}{*}{$\psi$} & \multirow{3}{*}{$\begin{array}{l}n \\
\text { error } \\
\tau(\psi) \mid \tau(A)\end{array}$} & \multicolumn{8}{|c|}{200} & \multicolumn{8}{|c|}{500} & \multicolumn{8}{|c|}{1000} \\
\hline & & \multicolumn{4}{|c|}{ IRAE(x100) } & \multicolumn{4}{|c|}{$\operatorname{ISE}(x 10000)$} & \multicolumn{4}{|c|}{ IRAE(x100) } & \multicolumn{4}{|c|}{$\operatorname{ISE}(x 10000)$} & \multicolumn{4}{|c|}{ IRAE $(x 100)$} & \multicolumn{4}{|c|}{$\operatorname{ISE}(x 10000)$} \\
\hline & & $1 / 5$ & $2 / 5$ & $3 / 5$ & $4 / 5$ & $1 / 5$ & $2 / 5$ & $3 / 5$ & $4 / 5$ & $1 / 5$ & $2 / 5$ & $3 / 5$ & $4 / 5$ & $1 / 5$ & $2 / 5$ & $3 / 5$ & $4 / 5$ & $1 / 5$ & $2 / 5$ & $3 / 5$ & $4 / 5$ & $1 / 5$ & $2 / 5$ & $3 / 5$ & $4 / 5$ \\
\hline \multirow[t]{4}{*}{ Clayton } & $1 / 5$ & 6.6 & 3.23 & 1.03 & 0.17 & 2.22 & 1.72 & 1.04 & 0.4 & 2.52 & 1.23 & 0.4 & 0.06 & 1.38 & 1.06 & 0.64 & 0.24 & 1.42 & 0.7 & 0.22 & 0.03 & 1.03 & 0.8 & 0.48 & 0.17 \\
\hline & $2 / 5$ & 8.68 & 3.9 & 1.2 & 0.2 & 2.54 & 1.88 & 1.11 & 0.44 & 3.25 & 1.47 & 0.46 & 0.07 & 1.57 & 1.16 & 0.69 & 0.26 & 1.78 & 0.81 & 0.25 & 0.03 & 1.17 & 0.86 & 0.51 & 0.19 \\
\hline & $3 / 5$ & 10.01 & 4.4 & 1.51 & 0.36 & 2.7 & 1.96 & 1.23 & 0.6 & 4.12 & 1.75 & 0.55 & 0.1 & 1.74 & 1.24 & 0.74 & 0.32 & 2.22 & 0.95 & 0.3 & 0.05 & 1.3 & 0.94 & 0.56 & 0.22 \\
\hline & $4 / 5$ & 16.01 & 7.96 & 3.49 & 1.08 & 3.4 & 2.62 & 1.9 & 1.12 & 5.66 & 2.58 & 0.96 & 0.26 & 2 & 1.48 & 0.99 & 0.55 & 2.89 & 1.26 & 0.44 & 0.11 & 1.46 & 1.07 & 0.68 & 0.35 \\
\hline \multirow{4}{*}{ Frank } & $1 / 5$ & 6.49 & 3.11 & 1 & 0.15 & 2.23 & 1.7 & 1.03 & 0.38 & 2.48 & 1.22 & 0.38 & 0.05 & 1.36 & 1.05 & 0.63 & 0.23 & 1.37 & 0.68 & 0.21 & 0.03 & 1.02 & 0.79 & 0.47 & 0.16 \\
\hline & $2 / 5$ & 7.16 & 3.19 & 1.01 & 0.16 & 2.31 & 1.69 & 1.01 & 0.39 & 2.71 & 1.21 & 0.37 & 0.05 & 1.44 & 1.06 & 0.62 & 0.23 & 1.5 & 0.66 & 0.19 & 0.03 & 1.07 & 0.78 & 0.45 & 0.16 \\
\hline & $3 / 5$ & 6.94 & 2.97 & 0.94 & 0.18 & 2.31 & 1.66 & 0.99 & 0.42 & 2.78 & 1.17 & 0.36 & 0.06 & 1.44 & 1.03 & 0.61 & 0.23 & 1.51 & 0.64 & 0.19 & 0.03 & 1.07 & 0.76 & 0.45 & 0.16 \\
\hline & $4 / 5$ & 7.56 & 3.3 & 1.24 & 0.39 & 2.36 & 1.72 & 1.13 & 0.65 & 2.61 & 1.12 & 0.38 & 0.09 & 1.39 & 1 & 0.63 & 0.3 & 1.42 & 0.6 & 0.18 & 0.03 & 1.04 & 0.74 & 0.45 & 0.19 \\
\hline \multirow[t]{4}{*}{ Gumbel } & $1 / 5$ & 7.59 & 3.46 & 1.09 & 0.16 & 2.39 & 1.77 & 1.06 & 0.4 & 3.06 & 1.42 & 0.43 & 0.06 & 1.52 & 1.14 & 0.67 & 0.24 & 1.66 & 0.76 & 0.23 & 0.03 & 1.13 & 0.84 & 0.49 & 0.17 \\
\hline & $2 / 5$ & 8.95 & 3.78 & 1.19 & 0.19 & 2.61 & 1.87 & 1.12 & 0.43 & 3.7 & 1.57 & 0.47 & 0.07 & 1.67 & 1.2 & 0.69 & 0.26 & 1.95 & 0.83 & 0.25 & 0.03 & 1.22 & 0.87 & 0.51 & 0.18 \\
\hline & $3 / 5$ & 10.13 & 4.31 & 1.39 & 0.3 & 2.75 & 1.96 & 1.19 & 0.55 & 4.15 & 1.69 & 0.51 & 0.08 & 1.75 & 1.22 & 0.72 & 0.29 & 2.2 & 0.9 & 0.27 & 0.04 & 1.3 & 0.92 & 0.53 & 0.2 \\
\hline & $4 / 5$ & 13.4 & 6.17 & 2.48 & 0.75 & 3.1 & 2.33 & 1.6 & 0.93 & 4.77 & 2.06 & 0.74 & 0.19 & 1.88 & 1.35 & 0.88 & 0.46 & 2.54 & 1.07 & 0.35 & 0.07 & 1.39 & 1 & 0.61 & 0.28 \\
\hline \multirow[t]{4}{*}{ Joe } & $1 / 5$ & 7.99 & 3.64 & 1.14 & 0.18 & 2.45 & 1.82 & 1.08 & 0.42 & 3.33 & 1.49 & 0.45 & 0.06 & 1.59 & 1.16 & 0.68 & 0.24 & 1.77 & 0.78 & 0.24 & 0.03 & 1.16 & 0.85 & 0.5 & 0.18 \\
\hline & $2 / 5$ & 9.54 & 4.09 & 1.28 & 0.22 & 2.7 & 1.93 & 1.15 & 0.47 & 4.2 & 1.74 & 0.52 & 0.08 & 1.77 & 1.25 & 0.73 & 0.27 & 2.17 & 0.9 & 0.26 & 0.04 & 1.28 & 0.91 & 0.53 & 0.19 \\
\hline & $3 / 5$ & 11.67 & 5.13 & 1.71 & 0.37 & 2.94 & 2.13 & 1.31 & 0.62 & 4.94 & 2.01 & 0.62 & 0.11 & 1.9 & 1.33 & 0.78 & 0.34 & 2.56 & 1.04 & 0.31 & 0.05 & 1.39 & 0.98 & 0.57 & 0.23 \\
\hline & $4 / 5$ & 18.06 & 8.65 & 3.74 & 1.11 & 3.62 & 2.75 & 1.98 & 1.14 & 6.32 & 2.77 & 1.03 & 0.29 & 2.13 & 1.56 & 1.04 & 0.59 & 3.21 & 1.38 & 0.47 & 0.11 & 1.56 & 1.13 & 0.71 & 0.36 \\
\hline
\end{tabular}

Table 8: Average Integrated relative absolute error (IRAEx100) and Integrated squared error (ISEx10000) of $A_{n, c}^{\mathrm{CFG}}$ for 2-dimensional Archimax copula $C_{\psi, A}$ samples of size $n \in\{200,500,1000\}$. The Pickands dependence function $A$ is $\mathbf{L G}$ with four choices of parameters so that $\tau(A) \in\{1 / 5,2 / 5,3 / 5,4 / 5\}$, where $\tau(A)=\tau\left(C_{A}\right)$ is Kendall's tau of the bivariate extreme-value copula $C_{A}$. There are four choices for the Archimedean generator $\psi$, Clayton, Frank, Gumbel and Joe, each with four parameter choices so that $\tau(\psi) \in\{1 / 5,2 / 5,3 / 5,4 / 5\}$, where $\tau(\psi)=\tau\left(C_{\psi}\right)$ is Kendall's tau of the bivariate Archimedean copula $C_{\psi}$. There are 1000 Monte Carlo replicates. 


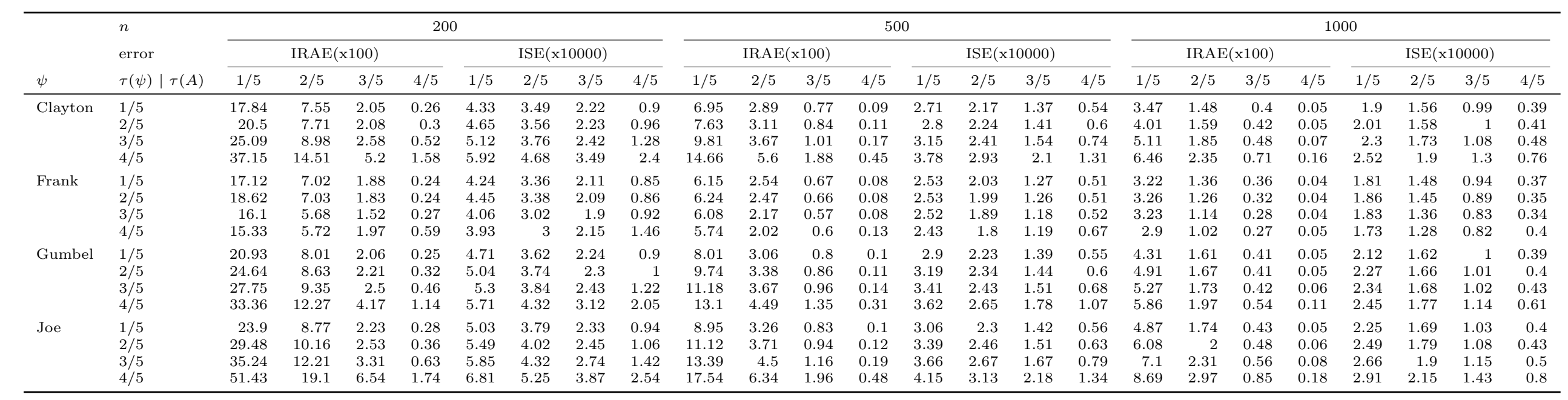

Table 9: Average Integrated relative absolute error (IRAEx100) and Integrated squared error (ISEx10000) of $A_{n, c}^{\mathrm{CFG}}$ and for 4-dimensional Archimax copula $C_{\psi, A}$ samples of size $n \in\{200,500,1000\}$. The Pickands dependence function $A$ is $\mathbf{L G}$ with four choices of parameters so that $\tau(A) \in$ $\{1 / 5,2 / 5,3 / 5,4 / 5\}$, where $\tau(A)=\tau\left(C_{A}\right)$ is Kendall's tau of the corresponding bivariate extreme-value copula $C_{A^{(2)}}$. $A^{(2)}$ is a 2-dimensional $\mathbf{L G}$ Pickands dependence function with the same parameter as $A$. There are four choices for the Archimedean generator $\psi$, Clayton, Frank, Gumbel and Joe, each with four parameter choices so that $\tau(\psi) \in\{1 / 5,2 / 5,3 / 5,4 / 5\}$, where $\tau(\psi)=\tau\left(C_{\psi}\right)$ is Kendall's tau of the bivariate Archimedean copula $C_{\psi}$. There are 1000 Monte Carlo replicates. 


\begin{tabular}{|c|c|c|c|c|c|c|c|c|c|c|c|c|c|c|c|c|c|c|c|c|c|c|c|c|c|}
\hline \multirow[b]{3}{*}{$\psi$} & \multirow{3}{*}{$\begin{array}{l}n \\
\text { error } \\
\tau(\psi) \mid \tau(A)\end{array}$} & \multicolumn{8}{|c|}{200} & \multicolumn{8}{|c|}{500} & \multicolumn{8}{|c|}{1000} \\
\hline & & \multicolumn{4}{|c|}{ IRAE $(x 100)$} & \multicolumn{4}{|c|}{$\operatorname{ISE}(x 10000)$} & \multicolumn{4}{|c|}{ IRAE(x100) } & \multicolumn{4}{|c|}{$\operatorname{ISE}(x 10000)$} & \multicolumn{4}{|c|}{ IRAE $(x 100)$} & \multicolumn{4}{|c|}{$\operatorname{ISE}(x 10000)$} \\
\hline & & $1 / 5$ & $2 / 5$ & $3 / 5$ & $4 / 5$ & $1 / 5$ & $2 / 5$ & $3 / 5$ & $4 / 5$ & $1 / 5$ & $2 / 5$ & $3 / 5$ & $4 / 5$ & $1 / 5$ & $2 / 5$ & $3 / 5$ & $4 / 5$ & $1 / 5$ & $2 / 5$ & $3 / 5$ & $4 / 5$ & $1 / 5$ & $2 / 5$ & $3 / 5$ & $4 / 5$ \\
\hline \multirow[t]{4}{*}{ Clayton } & $1 / 5$ & 32.88 & 9.78 & 1.97 & 0.2 & 7.1 & 5.74 & 3.73 & 1.6 & 13.8 & 4.34 & 0.87 & 0.08 & 4.59 & 3.82 & 2.47 & 1.04 & 6.53 & 2.03 & 0.4 & 0.04 & 3.11 & 2.6 & 1.68 & 0.71 \\
\hline & $2 / 5$ & 35.12 & 9.92 & 2.03 & 0.23 & 7.36 & 5.7 & 3.7 & 1.72 & 16.11 & 4.59 & 0.89 & 0.09 & 4.94 & 3.93 & 2.5 & 1.08 & 7.1 & 2.09 & 0.42 & 0.04 & 3.21 & 2.62 & 1.71 & 0.74 \\
\hline & $3 / 5$ & 43.17 & 11.68 & 2.48 & 0.38 & 8.1 & 6.22 & 4.1 & 2.19 & 18.48 & 5.05 & 1.01 & 0.12 & 5.22 & 4.07 & 2.63 & 1.28 & 8.9 & 2.38 & 0.47 & 0.05 & 3.65 & 2.81 & 1.8 & 0.83 \\
\hline & $4 / 5$ & 54.62 & 15.64 & 4.19 & 1.08 & 8.87 & 7.01 & 5.21 & 3.93 & 23.39 & 6.41 & 1.52 & 0.31 & 5.91 & 4.57 & 3.21 & 2.1 & 10.7 & 2.76 & 0.6 & 0.11 & 3.99 & 3.01 & 2.03 & 1.23 \\
\hline \multirow[t]{4}{*}{ Frank } & $1 / 5$ & 31.49 & 9.01 & 1.82 & 0.18 & 7 & 5.5 & 3.54 & 1.55 & 13.16 & 4.01 & 0.78 & 0.07 & 4.48 & 3.66 & 2.32 & 0.97 & 6.57 & 2.01 & 0.39 & 0.04 & 3.11 & 2.59 & 1.65 & 0.69 \\
\hline & $2 / 5$ & 28.42 & 7.96 & 1.62 & 0.18 & 6.61 & 5.15 & 3.34 & 1.51 & 11.96 & 3.41 & 0.67 & 0.07 & 4.24 & 3.34 & 2.14 & 0.92 & 5.9 & 1.65 & 0.32 & 0.03 & 2.97 & 2.35 & 1.5 & 0.63 \\
\hline & $3 / 5$ & 26.69 & 6.88 & 1.36 & 0.19 & 6.43 & 4.82 & 3.07 & 1.57 & 10.51 & 2.74 & 0.53 & 0.06 & 3.95 & 3 & 1.91 & 0.89 & 5.4 & 1.42 & 0.27 & 0.03 & 2.83 & 2.17 & 1.38 & 0.61 \\
\hline & $4 / 5$ & 21.7 & 5.79 & 1.46 & 0.4 & 5.67 & 4.3 & 3.1 & 2.39 & 8.29 & 2.13 & 0.47 & 0.09 & 3.47 & 2.62 & 1.78 & 1.14 & 4.06 & 1.05 & 0.22 & 0.03 & 2.43 & 1.85 & 1.22 & 0.67 \\
\hline \multirow[t]{4}{*}{ Gumbel } & $1 / 5$ & 40.74 & 10.81 & 2.05 & 0.21 & 7.89 & 6.02 & 3.77 & 1.64 & 17.62 & 4.68 & 0.88 & 0.08 & 5.1 & 3.93 & 2.47 & 1.04 & 8.53 & 2.23 & 0.41 & 0.04 & 3.56 & 2.72 & 1.7 & 0.7 \\
\hline & $2 / 5$ & 48.43 & 11.84 & 2.18 & 0.24 & 8.54 & 6.31 & 3.91 & 1.76 & 21.52 & 5.16 & 0.93 & 0.09 & 5.61 & 4.12 & 2.54 & 1.09 & 9.91 & 2.38 & 0.43 & 0.04 & 3.81 & 2.8 & 1.74 & 0.73 \\
\hline & $3 / 5$ & 52.22 & 12.45 & 2.36 & 0.35 & 8.74 & 6.37 & 3.99 & 2.12 & 23.46 & 5.45 & 0.98 & 0.11 & 5.85 & 4.22 & 2.61 & 1.22 & 10.61 & 2.49 & 0.45 & 0.05 & 3.94 & 2.86 & 1.77 & 0.79 \\
\hline & $4 / 5$ & 61.23 & 15.44 & 3.7 & 0.85 & 9.08 & 6.79 & 4.88 & 3.47 & 24.49 & 5.89 & 1.25 & 0.22 & 5.96 & 4.35 & 2.9 & 1.78 & 11.09 & 2.68 & 0.54 & 0.08 & 4.04 & 2.99 & 1.95 & 1.09 \\
\hline \multirow[t]{4}{*}{ Joe } & $1 / 5$ & 47.39 & 11.98 & 2.23 & 0.22 & 8.46 & 6.3 & 3.91 & 1.68 & 20.08 & 5.01 & 0.93 & 0.09 & 5.45 & 4.05 & 2.54 & 1.08 & 10.08 & 2.47 & 0.45 & 0.04 & 3.85 & 2.85 & 1.76 & 0.73 \\
\hline & $2 / 5$ & 59.62 & 14.23 & 2.59 & 0.29 & 9.27 & 6.76 & 4.16 & 1.89 & 25.61 & 5.85 & 1.04 & 0.1 & 6.14 & 4.41 & 2.71 & 1.18 & 12.37 & 2.82 & 0.49 & 0.05 & 4.18 & 3.01 & 1.84 & 0.79 \\
\hline & $3 / 5$ & 71.83 & 16.91 & 3.2 & 0.45 & 9.86 & 7.13 & 4.51 & 2.39 & 29.34 & 6.66 & 1.19 & 0.14 & 6.51 & 4.67 & 2.87 & 1.38 & 13.83 & 3.13 & 0.56 & 0.06 & 4.42 & 3.16 & 1.95 & 0.9 \\
\hline & $4 / 5$ & 95.53 & 24.21 & 5.59 & $\begin{array}{l}1.19 \\
\text { nat }\end{array}$ & 10.97 & 8.3 & 5.97 & 4.15 & 34.69 & 8.39 & 1.81 & 0.34 & 6.93 & 5.1 & 3.47 & 2.2 & 15.34 & 3.7 & 0.76 & 0.13 & 4.64 & 3.42 & 2.28 & 1.35 \\
\hline
\end{tabular}

Table 10: Average Integrated relative absolute error (IRAEx100) and Integrated squared error (ISEx10000) of $A_{n, c}^{\mathrm{CFG}}$ and for 10-dimensional Archimax copula $C_{\psi, A}$ samples of size $n \in\{200,500,1000\}$. The Pickands dependence function $A$ is $\mathbf{L G}$ with four choices of parameters so that $\tau(A) \in$ $\{1 / 5,2 / 5,3 / 5,4 / 5\}$, where $\tau(A)=\tau\left(C_{A}\right)$ is Kendall's tau of the corresponding bivariate extreme-value copula $C_{A^{(2)}}$. $A^{(2)}$ is a 2 -dimensional LG Pickands dependence function with the same parameter as $A$. There are four choices for the Archimedean generator $\psi$, Clayton, Frank, Gumbel and Joe, each with four parameter choices so that $\tau(\psi) \in\{1 / 5,2 / 5,3 / 5,4 / 5\}$, where $\tau(\psi)=\tau\left(C_{\psi}\right)$ is Kendall's tau of the bivariate Archimedean copula $C_{\psi}$. There are 1000 Monte Carlo replicates. 


\begin{tabular}{|c|c|c|c|c|c|c|c|c|c|c|c|c|c|c|c|c|c|c|c|c|c|c|c|c|c|}
\hline \multirow[b]{3}{*}{$d$} & \multirow{3}{*}{$\begin{array}{l}n \\
\text { error } \\
\lambda_{U}(\psi, A) \mid \lambda_{U}(A)\end{array}$} & \multicolumn{8}{|c|}{200} & \multicolumn{8}{|c|}{500} & \multicolumn{8}{|c|}{1000} \\
\hline & & \multicolumn{4}{|c|}{ IRAE(x100) } & \multicolumn{4}{|c|}{ ISE(x10000) } & \multicolumn{4}{|c|}{ IRAE(x100) } & \multicolumn{4}{|c|}{$\operatorname{ISE}(x 10000)$} & \multicolumn{4}{|c|}{ IRAE(x100) } & \multicolumn{4}{|c|}{ ISE(x10000) } \\
\hline & & $1 / 5$ & $2 / 5$ & $3 / 5$ & $4 / 5$ & $1 / 5$ & $2 / 5$ & $3 / 5$ & $4 / 5$ & $1 / 5$ & $2 / 5$ & $3 / 5$ & $4 / 5$ & $1 / 5$ & $2 / 5$ & $3 / 5$ & $4 / 5$ & $1 / 5$ & $2 / 5$ & $3 / 5$ & $4 / 5$ & $1 / 5$ & $2 / 5$ & $3 / 5$ & $4 / 5$ \\
\hline \multirow[t]{4}{*}{2} & $\begin{array}{l}5 / 10 \\
6 / 10\end{array}$ & $\begin{array}{r}9.34 \\
10.37\end{array}$ & $\begin{array}{l}4.63 \\
5.04\end{array}$ & & & $\begin{array}{r}2.6 \\
2.74\end{array}$ & $\begin{array}{l}1.98 \\
2.07\end{array}$ & & & $\begin{array}{l}3.91 \\
4.45\end{array}$ & $\begin{array}{l}1.83 \\
2.08\end{array}$ & & & $\begin{array}{l}1.67 \\
1.78\end{array}$ & $\begin{array}{l}1.26 \\
1.33\end{array}$ & & & $\begin{array}{l}2.06 \\
2.31\end{array}$ & $\begin{array}{r}1 \\
1.1\end{array}$ & & & $\begin{array}{r}1.22 \\
1.3\end{array}$ & $\begin{array}{l}.92 \\
.98\end{array}$ & & \\
\hline & $7 / 10$ & 11.38 & 5.49 & 1.94 & & 2.88 & 2.16 & 1.38 & & 5.02 & 2.35 & .77 & & $\begin{array}{l}1.89 \\
\end{array}$ & 1.41 & .88 & & $\begin{array}{r}2.61 \\
2.6\end{array}$ & $\begin{array}{l}1.1 \\
1.22\end{array}$ & .41 & & $\begin{array}{l}1.0 \\
1.37\end{array}$ & $\begin{array}{r}.30 \\
1.02\end{array}$ & .64 & \\
\hline & $8 / 10$ & 13.18 & 6.11 & 2.11 & & 3.07 & 2.26 & 1.45 & & 5.64 & 2.63 & .88 & & 1.98 & 1.48 & .93 & & 2.95 & 1.37 & .45 & & 1.45 & 1.08 & .67 & \\
\hline & $9 / 10$ & 17.3 & 8.22 & 2.73 & .39 & 3.48 & 2.59 & 1.61 & .63 & 6.65 & 3.11 & 1.03 & .14 & 2.13 & 1.59 & .99 & .38 & 3.47 & 1.62 & .53 & .07 & 1.58 & 1.17 & .73 & .28 \\
\hline \multirow[t]{5}{*}{2} & $5 / 10$ & 29.13 & 11.76 & & & 5.25 & 4.02 & & & 10.98 & 4.37 & & & 3.2 & 2.44 & & & 5.98 & 2.38 & & & 2.36 & 1.81 & & \\
\hline & $6 / 10$ & 33.02 & 13.18 & & & 5.55 & 4.27 & & & 12.59 & 4.88 & & & 3.42 & 2.59 & & & 6.8 & 2.64 & & & 2.5 & 1.9 & & \\
\hline & $7 / 10$ & 37.03 & 14.6 & 4 & & 5.82 & 4.47 & 2.89 & & 14.08 & 5.45 & 1.52 & & 3.6 & 2.74 & 1.76 & & 7.71 & 2.95 & .8 & & 2.65 & 2.01 & 1.29 & \\
\hline & $8 / 10$ & 41.83 & 16.44 & 4.47 & & 6.08 & 4.66 & 3.03 & & 16.22 & 6.12 & 1.7 & & 3.83 & 2.9 & 1.88 & & 8.68 & 3.31 & .89 & & 2.79 & 2.1 & 1.36 & \\
\hline & $9 / 10$ & 54.45 & 20.81 & 5.78 & .65 & 6.73 & 5.1 & 3.34 & 1.39 & 19.48 & 7.63 & 2.12 & .24 & 4.16 & 3.17 & 2.07 & .86 & 10.12 & 3.88 & 1.05 & .12 & 2.97 & 2.25 & 1.45 & .6 \\
\hline \multirow[t]{5}{*}{10} & $5 / 10$ & 63.58 & 17.87 & & & 8.88 & 6.64 & & & 27.19 & 7.63 & & & 5.74 & 4.32 & & & 13.72 & 3.82 & & & 4.06 & 3.07 & & \\
\hline & $6 / 10$ & 73.1 & 20.78 & & & 9.42 & 7.14 & & & 31.54 & 8.71 & & & 6.16 & 4.58 & & & 15.62 & 4.31 & & & 4.28 & 3.24 & & \\
\hline & $7 / 10$ & 82.19 & 23.37 & 4.53 & & 9.89 & 7.51 & 4.78 & & 35.6 & 9.87 & 1.9 & & 6.55 & 4.87 & 3.11 & & 17.26 & 4.84 & .93 & & 4.47 & 3.39 & 2.19 & \\
\hline & $8 / 10$ & 94.21 & 27.01 & 5.21 & & 10.34 & 7.87 & 5.12 & & 39.37 & 11.02 & 2.17 & & 6.88 & 5.17 & 3.34 & & 18.7 & 5.33 & 1.04 & & 4.65 & 3.53 & 2.29 & \\
\hline & $9 / 10$ & 112.8 & 31.95 & 6.53 & .56 & 10.93 & 8.37 & 5.52 & 2.44 & 44.77 & 12.64 & 2.52 & .22 & 7.19 & 5.46 & 3.58 & 1.58 & 20.29 & 5.89 & 1.2 & .11 & 4.85 & 3.7 & 2.44 & 1.08 \\
\hline
\end{tabular}

Table 11: Average Integrated relative absolute error (IRAEx100) and Integrated squared error (ISEx10000) of $A_{n, c}^{\mathrm{CFG}}$ and for $d$-dimensional Archimax copula $C_{\psi, A}$ samples of size $n \in\{200,500,1000\}$ for dimensions $d \in\{2,4,10\}$. The Pickands dependence function $A$ is $\mathbf{L G}$ with four choices of parameters so that $\lambda_{U}(A) \in\{1 / 5,2 / 5,3 / 5,4 / 5\}$, where $\lambda_{U}(A)=\lambda_{U}\left(C_{A}\right)$ is the upper tail dependence coefficient of the corresponding bivariate extremevalue copula $C_{A^{(2)}} . A^{(2)}$ is a 2 -dimensional LG Pickands dependence function with the same parameter as $A$. The Archimedean generator $\psi$ is Joe and $\lambda_{U}(\psi, A) \in\{5 / 10,6 / 10,7 / 10,8 / 10,9 / 10\}$, where $\lambda_{U}(\psi, A)=\lambda_{U}\left(C_{\psi, A}\right)$ is the upper tail coefficient of the bivariate Archimax copula $C_{\psi, A^{(2)}}$. There are 1000 Monte Carlo replicates. 


\begin{tabular}{|c|c|c|c|c|c|c|c|c|c|c|c|c|c|c|c|c|c|c|c|c|c|c|c|c|c|}
\hline \multirow{3}{*}{$\begin{array}{l}\psi \\
d\end{array}$} & \multirow{3}{*}{$\begin{array}{l}n \\
\text { error } \\
\lambda_{L}(\psi, A) \mid \eta_{L}(A)\end{array}$} & \multicolumn{8}{|c|}{200} & \multicolumn{8}{|c|}{500} & \multicolumn{8}{|c|}{1000} \\
\hline & & \multicolumn{4}{|c|}{ IRAE(x100) } & \multicolumn{4}{|c|}{ ISE(x10000) } & \multicolumn{4}{|c|}{ IRAE (x100) } & \multicolumn{4}{|c|}{$\operatorname{ISE}(x 10000)$} & \multicolumn{4}{|c|}{ IRAE(x100) } & \multicolumn{4}{|c|}{ ISE $(x 10000)$} \\
\hline & & 0.57 & 0.66 & 0.76 & 0.87 & 0.57 & 0.66 & 0.76 & 0.87 & 0.57 & 0.66 & 0.76 & 0.87 & 0.57 & 0.66 & 0.76 & 0.87 & 0.57 & 0.66 & 0.76 & 0.87 & 0.57 & 0.66 & 0.76 & 0.87 \\
\hline \multirow[t]{4}{*}{2} & $1 / 5$ & 6.39 & 3.24 & 1.06 & 0.15 & 2.17 & 1.71 & 1.05 & 0.39 & 2.48 & 1.26 & 0.4 & 0.05 & 1.36 & 1.07 & 0.64 & 0.23 & 1.37 & 0.69 & 0.22 & 0.03 & 1.02 & 0.8 & 0.48 & 0.17 \\
\hline & $2 / 5$ & 6.81 & 3.23 & 1.04 & 0.16 & 2.26 & 1.71 & 1.04 & 0.39 & 2.78 & 1.31 & 0.39 & 0.05 & 1.46 & 1.09 & 0.65 & 0.23 & 1.52 & 0.68 & 0.22 & 0.03 & 1.07 & 0.8 & 0.48 & 0.17 \\
\hline & $3 / 5$ & 8.33 & 3.47 & 1.07 & 0.15 & 2.54 & 1.79 & 1.06 & 0.39 & 3.24 & 1.48 & 0.4 & 0.06 & 1.57 & 1.16 & 0.65 & 0.24 & 1.71 & 0.73 & 0.23 & 0.03 & 1.14 & 0.81 & 0.49 & 0.17 \\
\hline & $4 / 5$ & 9.96 & 4.07 & 1.22 & 0.17 & 2.71 & 1.9 & 1.12 & 0.4 & 3.79 & 1.5 & 0.46 & 0.06 & 1.69 & 1.17 & 0.69 & 0.24 & 2.07 & 0.84 & 0.24 & 0.03 & 1.26 & 0.88 & 0.5 & 0.17 \\
\hline \multirow[t]{3}{*}{4} & $1 / 5$ & 16.81 & 6.99 & 1.92 & 0.23 & 4.22 & 3.4 & 2.16 & 0.86 & 6.52 & 2.88 & 0.77 & 0.09 & 2.62 & 2.15 & 1.36 & 0.53 & 3.41 & 1.45 & 0.39 & 0.04 & 1.88 & 1.53 & 0.97 & 0.37 \\
\hline & $2 / 5$ & 3.08 & 7.21 & 2 & 0.23 & 4.37 & 3.42 & 2.18 & 0.85 & 6.73 & 2.89 & 0.78 & 0.09 & 2.64 & 2.17 & 1.37 & 0.54 & 3.5 & 1.5 & 0.38 & 0.04 & 1.9 & 1.56 & 0.96 & 0.38 \\
\hline & $3 / 5$ & 20.02 & 7.42 & 2.07 & 0.25 & 4.58 & 3.46 & 2.22 & 0.89 & 7.9 & 2.95 & 0.75 & 0.09 & 2.84 & 2.18 & 1.35 & 0.53 & 3.73 & 1.46 & 0.39 & 0.04 & 1.96 & 1.53 & 0.97 & 0.38 \\
\hline \multirow[t]{3}{*}{10} & $1 / 5$ & $\begin{array}{r}33.23 \\
34 .\end{array}$ & 10.06 & 2 & 0.19 & $\begin{array}{l}7.15 \\
7.37\end{array}$ & $\begin{array}{r}5.8 \\
5.86\end{array}$ & $\begin{array}{l}3.72 \\
3.74\end{array}$ & 1.58 & $\begin{array}{r}12.8 \\
1381\end{array}$ & 4.15 & $\begin{array}{l}0.83 \\
0.79\end{array}$ & 0.08 & $\begin{array}{r}4.4 \\
\end{array}$ & $\begin{array}{r}3.7 \\
3.7\end{array}$ & $\begin{array}{r}2.4 \\
2.35\end{array}$ & 1 & 6.54 & $\begin{array}{l}2.02 \\
1.97\end{array}$ & $\begin{array}{r}0.39 \\
0.49\end{array}$ & 0.04 & $\begin{array}{l}3.09 \\
3.12\end{array}$ & $\begin{array}{r}2.6 \\
\end{array}$ & $\begin{array}{l}1.63 \\
1.68\end{array}$ & $\begin{array}{r}0.7 \\
0.6\end{array}$ \\
\hline & $\begin{array}{l}2 / 5 \\
3 / 5\end{array}$ & $\begin{array}{r}34.7 \\
34.72\end{array}$ & $\begin{array}{l}10.12 \\
10.49\end{array}$ & $\begin{array}{r}2 \\
2.02\end{array}$ & $\begin{array}{r}0.2 \\
0.19\end{array}$ & $\begin{array}{l}7.37 \\
7.32\end{array}$ & $\begin{array}{l}5.86 \\
5.94\end{array}$ & $\begin{array}{l}3.74 \\
3.78\end{array}$ & $\begin{array}{l}1.61 \\
1.59\end{array}$ & $\begin{array}{l}13.81 \\
15.36\end{array}$ & $\begin{array}{l}4.17 \\
4.09\end{array}$ & $\begin{array}{l}0.79 \\
0.86\end{array}$ & $\begin{array}{l}0.08 \\
0.08\end{array}$ & $\begin{array}{l}4.57 \\
4.81\end{array}$ & $\begin{array}{l}3.77 \\
3.71\end{array}$ & $\begin{array}{l}2.35 \\
2.46\end{array}$ & $\begin{array}{r}1.01 \\
1\end{array}$ & $\begin{array}{l}6.68 \\
7.11\end{array}$ & $\begin{array}{l}1.97 \\
2.08\end{array}$ & $\begin{array}{r}0.4 \\
0.42\end{array}$ & $\begin{array}{l}0.04 \\
0.04\end{array}$ & $\begin{array}{l}3.12 \\
3.22\end{array}$ & $\begin{array}{l}2.56 \\
2.62\end{array}$ & $\begin{array}{l}1.68 \\
1.71\end{array}$ & $\begin{array}{l}0.69 \\
0.71\end{array}$ \\
\hline & $4 / 5$ & 39.04 & 10.01 & 1.94 & 0.21 & 7.68 & 5.8 & 3.7 & 1.63 & 17.48 & 4.89 & 0.93 & 0.08 & 5.1 & 4.05 & 2.56 & 1.05 & 8.37 & 2.17 & 0.42 & 0.04 & 3.49 & 2.67 & 1.72 & 0.7 \\
\hline
\end{tabular}

Table 12: Average Integrated relative absolute error (IRAEx100) and Integrated squared error (ISEx10000) of $A_{n, c}^{\mathrm{CFG}}$ and $A_{n, c}^{\mathrm{P}}$ for $d$-dimensional Archimax copula $C_{\psi, A}$ samples of size $n \in\{200,500,1000\}$ for dimensions $d \in\{2,4,10\}$. The Pickands dependence function $A$ is $\mathbf{L G}$ with four choices of parameters so that $\eta_{L}(A) \in\{1 / 5,2 / 5,3 / 5,4 / 5\}$, where $\eta_{L}(A)=\eta_{L}\left(C_{A}\right)$ is the index of lower tail dependence [34] of the corresponding bivariate extreme-value copula $C_{A^{(2)}}$. $\quad A^{(2)}$ is a 2-dimensional LG Pickands dependence function with the same parameter as $A$. The Archimedean generator $\psi$ is Clayton and $\lambda_{L}(\psi, A) \in\{5 / 10,6 / 10,7 / 10,8 / 10,9 / 10\}$, where $\lambda_{L}(\psi, A)=\lambda_{L}\left(C_{\psi, A}\right)$ is the lower tail coefficient of the bivariate Archimax copula $C_{\psi, A(2)}$. There are 1000 Monte Carlo replicates. 


\begin{tabular}{|c|c|c|c|c|c|c|c|c|c|c|c|c|c|}
\hline \multirow[b]{3}{*}{$\psi$} & \multirow{3}{*}{$\begin{array}{l}\text { error } \\
n \\
\tau(\psi) \mid A\end{array}$} & \multicolumn{6}{|c|}{ IRAE(x100) } & \multicolumn{6}{|c|}{ ISE $(x 10000)$} \\
\hline & & \multicolumn{2}{|c|}{200} & \multicolumn{2}{|c|}{500} & \multicolumn{2}{|c|}{1000} & \multicolumn{2}{|c|}{200} & \multicolumn{2}{|c|}{500} & \multicolumn{2}{|c|}{1000} \\
\hline & & $\overline{\mathrm{DSM}}$ & $\overline{L G}$ & $\overline{\mathrm{DSM}}$ & $\overline{L G}$ & $\overline{\mathrm{DSM}}$ & $\overline{L G}$ & $\overline{\mathrm{DSM}}$ & $\overline{L G}$ & $\overline{\mathrm{DSM}}$ & $\overline{L G}$ & $\overline{\mathrm{DSM}}$ & LG \\
\hline \multirow[t]{4}{*}{ Clayton } & $1 / 5$ & 0.99 & 0.9 & 0.61 & 0.55 & 0.42 & 0.4 & 1 & 0.75 & 0.39 & 0.28 & 0.18 & 0.14 \\
\hline & $2 / 5$ & 1.02 & 0.96 & 0.64 & 0.59 & 0.44 & 0.41 & 1.05 & 0.87 & 0.41 & 0.33 & 0.19 & 0.16 \\
\hline & $3 / 5$ & 1.16 & $\begin{array}{l}1.09 \\
\text { S }\end{array}$ & 0.71 & 0.65 & 0.47 & 0.45 & 1.33 & 1.18 & 0.5 & 0.41 & 0.22 & 0.19 \\
\hline & $4 / 5$ & 1.76 & 1.66 & 0.94 & 0.92 & 0.61 & 0.56 & 2.89 & 2.56 & 0.82 & 0.78 & 0.34 & 0.29 \\
\hline \multirow[t]{4}{*}{ Frank } & $1 / 5$ & 0.95 & 0.88 & 0.58 & 0.56 & 0.41 & 0.38 & 0.93 & 0.73 & 0.35 & 0.29 & 0.17 & 0.14 \\
\hline & $2 / 5$ & 0.94 & 0.86 & 0.57 & 0.54 & 0.4 & 0.38 & 0.9 & 0.7 & 0.33 & 0.28 & 0.16 & 0.13 \\
\hline & $3 / 5$ & 0.93 & 0.88 & 0.57 & 0.54 & 0.38 & 0.37 & 0.88 & 0.74 & 0.33 & 0.27 & 0.15 & 0.13 \\
\hline & $4 / 5$ & 1.14 & 1 & 0.62 & 0.56 & 0.39 & 0.37 & 1.22 & 0.94 & 0.38 & 0.29 & 0.15 & 0.13 \\
\hline \multirow[t]{4}{*}{ Gumbel } & $1 / 5$ & 1 & 0.91 & 0.61 & 0.57 & 0.41 & 0.39 & 1.04 & 0.78 & 0.38 & 0.31 & 0.17 & 0.15 \\
\hline & $2 / 5$ & 1.03 & 0.92 & 0.63 & 0.59 & 0.42 & 0.41 & 1.07 & 0.82 & 0.41 & 0.32 & 0.18 & 0.16 \\
\hline & $3 / 5$ & 1.12 & 1.01 & 0.67 & 0.63 & 0.45 & 0.43 & 1.23 & 0.99 & 0.44 & 0.37 & 0.19 & 0.17 \\
\hline & $4 / 5$ & 1.56 & 1.38 & 0.84 & 0.8 & 0.52 & $\begin{array}{r}0.75 \\
0.5\end{array}$ & 2.21 & 1.77 & 0.66 & 0.59 & 0.26 & 0.23 \\
\hline \multirow[t]{4}{*}{ Joe } & $1 / 5$ & 1.02 & 0.92 & 0.64 & 0.57 & 0.41 & 0.41 & 1.09 & 0.81 & 0.41 & 0.31 & 0.17 & 0.16 \\
\hline & $2 / 5$ & 1.12 & 1 & 0.68 & 0.61 & 0.44 & 0.44 & 1.29 & 0.95 & 0.46 & 0.35 & 0.2 & 0.18 \\
\hline & $3 / 5$ & 1.27 & 1.18 & 0.76 & 0.66 & 0.48 & 0.47 & 1.63 & 1.35 & 0.56 & 0.42 & 0.23 & 0.21 \\
\hline & $4 / 5$ & 1.91 & 1.81 & 1.03 & 0.94 & 0.61 & 0.6 & 3.43 & 2.98 & 0.98 & 0.8 & 0.35 & 0.34 \\
\hline
\end{tabular}

Table 13: Average Integrated relative absolute error (IRAEx100) and Integrated squared error (ISEx10000) of $A_{n, c}^{\mathrm{CFG}}$ for 2-dimensional Archimax copula $C_{\psi, A}$ samples of size $n \in\{200,500,1000\}$. The Pickands dependence function $A$ is either DSM or LG. Parameters for the DSM case are reported in Equation (11.1). The parameter in LG is set $\varrho=2.87$ so that the averaged pairwise Kendall's tau of both Pickands dependence functions is approximately equal to 0.65 . There are four choices for the Archimedean generator $\psi$, Clayton, Frank, Gumbel and Joe, each with four parameter choices so that $\tau(\psi) \in\{1 / 5,2 / 5,3 / 5,4 / 5\}$, where $\tau(\psi)=\tau\left(C_{\psi}\right)$ is Kendall's tau of the bivariate Archimedean copula $C_{\psi}$. There are 1000 Monte Carlo replicates. 


\begin{tabular}{|c|c|c|c|c|c|c|c|c|c|c|c|c|c|}
\hline \multirow[b]{3}{*}{$\psi$} & \multirow{3}{*}{$\begin{array}{l}\text { error } \\
n \\
\tau(\psi) \mid A\end{array}$} & \multicolumn{6}{|c|}{ IRAE(x100) } & \multicolumn{6}{|c|}{ ISE(x10000) } \\
\hline & & \multicolumn{2}{|c|}{200} & \multicolumn{2}{|c|}{500} & \multicolumn{2}{|c|}{1000} & \multicolumn{2}{|c|}{200} & \multicolumn{2}{|c|}{500} & \multicolumn{2}{|c|}{1000} \\
\hline & & $\overline{\mathrm{DSM}}$ & LG & $\overline{\mathrm{DSM}}$ & LG & $\overline{\mathrm{DSM}}$ & LG & DSM & $\mathrm{LG}$ & $\overline{D S M}$ & $\mathrm{LG}$ & $\overline{\mathrm{DSM}}$ & LG \\
\hline \multirow{4}{*}{ Clayton } & $1 / 5$ & 3.59 & 2.54 & 2.31 & 1.63 & 1.63 & 1.16 & 10.98 & 2.92 & 4.6 & 1.22 & 2.26 & 0.63 \\
\hline & $2 / 5$ & 4.06 & 2.63 & 2.57 & 1.69 & 1.9 & 1.16 & 14.02 & 3.17 & 5.77 & 1.31 & 3.08 & 0.63 \\
\hline & $3 / 5$ & 4.68 & 2.86 & 2.98 & 1.81 & 2.15 & 1.28 & 18.66 & 3.86 & 7.67 & 1.54 & 3.98 & 0.77 \\
\hline & $4 / 5$ & 5.89 & 3.84 & 3.69 & 2.28 & 2.57 & 1.46 & 30.67 & 7.22 & 11.87 & 2.48 & 5.83 & 0.99 \\
\hline \multirow[t]{4}{*}{ Frank } & $1 / 5$ & 3.5 & 2.47 & 2.26 & 1.61 & 1.61 & 1.08 & 10.3 & 2.81 & 4.39 & 1.19 & 2.25 & 0.54 \\
\hline & $2 / 5$ & 3.73 & 2.35 & 2.4 & $\begin{array}{l}1.49 \\
\text { S }\end{array}$ & 1.71 & 1.07 & 11.77 & 2.55 & 4.96 & 1.02 & 2.51 & 0.53 \\
\hline & $3 / 5$ & 3.9 & 2.2 & 2.45 & $\begin{array}{l}1.42 \\
\text { S }\end{array}$ & 1.8 & 1 & 12.81 & 2.23 & 5.2 & 0.94 & 2.79 & 0.45 \\
\hline & $4 / 5$ & 3.97 & 2.37 & 2.41 & 1.42 & 1.74 & 0.92 & 13.73 & 2.67 & 5.05 & 0.94 & 2.61 & 0.39 \\
\hline \multirow[t]{4}{*}{ Gumbel } & $1 / 5$ & 4.04 & 2.62 & 2.57 & 1.64 & 1.83 & 1.18 & 14.17 & 3.14 & 5.6 & 1.25 & 2.83 & 0.64 \\
\hline & $2 / 5$ & 4.52 & 2.68 & 2.9 & 1.68 & 2.04 & 1.17 & 18.11 & 3.4 & 7.16 & 1.31 & 3.55 & 0.64 \\
\hline & $3 / 5$ & 4.89 & 2.82 & 3.13 & 1.75 & 2.2 & 1.18 & 21.7 & 3.86 & 8.34 & 1.44 & 4.14 & 0.65 \\
\hline & $4 / 5$ & 5.61 & 3.42 & 3.38 & 1.99 & 2.36 & 1.28 & 28.32 & 5.71 & 10.1 & 1.92 & 4.84 & 0.78 \\
\hline \multirow[t]{4}{*}{ Joe } & $1 / 5$ & 4.39 & 2.62 & 2.72 & 1.66 & 1.94 & 1.16 & 16.65 & 3.23 & 6.29 & 1.27 & 3.24 & 0.64 \\
\hline & $2 / 5$ & 5.12 & 2.86 & 3.14 & 1.75 & 2.19 & 1.25 & 23.51 & 3.94 & 8.43 & 1.45 & 4.17 & 0.74 \\
\hline & $3 / 5$ & 5.61 & 3.27 & 3.43 & 1.91 & 2.39 & 1.34 & 29.86 & 5.36 & 10.25 & 1.74 & 5.02 & 0.86 \\
\hline & $4 / 5$ & 6.88 & 4.44 & 3.92 & 2.45 & 2.67 & 1.59 & 46.17 & 9.75 & 13.7 & 2.83 & 6.35 & 1.2 \\
\hline
\end{tabular}

Table 14: Average Integrated relative absolute error (IRAEx100) and Integrated squared error (ISEx10000) of $A_{n, c}^{\mathrm{CFG}}$ for 4-dimensional Archimax copula $C_{\psi, A}$ samples of size $n \in\{200,500,1000\}$. The Pickands dependence function $A$ is either DSM or LG. Parameters for the DSM case are reported in Equation (11.2). The parameter in LG is set $\varrho=2.17$ so that the averaged pairwise Kendall's tau of both Pickands dependence functions is approximately equal to 0.54 . There are four choices for the Archimedean generator $\psi$, Clayton, Frank, Gumbel and Joe, each with four parameter choices so that $\tau(\psi) \in\{1 / 5,2 / 5,3 / 5,4 / 5\}$, where $\tau(\psi)=\tau\left(C_{\psi}\right)$ is Kendall's tau of the bivariate Archimedean copula $C_{\psi}$. There are 1000 Monte Carlo replicates. 


\begin{tabular}{|c|c|c|c|c|c|c|c|c|c|c|c|c|c|}
\hline \multirow[b]{3}{*}{$\psi$} & \multirow{3}{*}{$\begin{array}{l}\text { error } \\
n \\
\tau(\psi) \mid A\end{array}$} & \multicolumn{6}{|c|}{$\operatorname{IRAE}(\mathrm{x} 100)$} & \multicolumn{6}{|c|}{ ISE $(x 10000)$} \\
\hline & & \multicolumn{2}{|c|}{200} & \multicolumn{2}{|c|}{500} & \multicolumn{2}{|c|}{1000} & \multicolumn{2}{|c|}{200} & \multicolumn{2}{|c|}{500} & \multicolumn{2}{|c|}{1000} \\
\hline & & $\overline{\mathrm{DSM}}$ & $\mathrm{LG}$ & $\overline{D S M}$ & $\mathrm{LG}$ & $\overline{\mathrm{DSM}}$ & $\mathrm{LG}$ & $\overline{\mathrm{DSM}}$ & LG & $\overline{D S M}$ & LG & $\overline{D S M}$ & LG \\
\hline \multirow[t]{4}{*}{ Clayton } & $1 / 5$ & 4.97 & 6.29 & 2.95 & 3.94 & 1.98 & 2.8 & 15.32 & 13.61 & 5.53 & 5.5 & 2.52 & 2.79 \\
\hline & $2 / 5$ & 5.95 & 6.21 & 3.72 & 4.19 & 2.55 & 2.87 & 22.12 & 13.15 & 8.87 & 6.12 & 4.23 & 3 \\
\hline & $3 / 5$ & 6.98 & 6.73 & 4.57 & 4.39 & 3.24 & 3.06 & 30.76 & 16.11 & 13.55 & 6.84 & 6.92 & 3.36 \\
\hline & $4 / 5$ & 8.71 & 7.74 & 5.47 & 4.66 & 3.92 & 3.45 & 51.59 & 23.07 & 19.81 & 8.14 & 10.37 & 4.37 \\
\hline \multirow[t]{4}{*}{ Frank } & $1 / 5$ & 4.84 & 5.93 & 2.92 & 3.75 & 1.96 & 2.64 & 14.75 & 12.45 & 5.4 & 4.98 & 2.52 & 2.46 \\
\hline & $2 / 5$ & 5.32 & 5.65 & 3.27 & 3.66 & 2.25 & 2.58 & 17.93 & 10.99 & 6.84 & 4.68 & 3.28 & 2.35 \\
\hline & $3 / 5$ & 5.38 & 5.15 & 3.35 & 3.33 & 2.42 & 2.34 & 18.69 & 9.37 & 7.3 & 3.89 & 3.81 & 1.94 \\
\hline & $4 / 5$ & 5.27 & 4.5 & 3.31 & 2.91 & 2.36 & 2.01 & 18.6 & 7.57 & 7.18 & 2.97 & 3.63 & 1.47 \\
\hline \multirow[t]{4}{*}{ Gumbel } & $1 / 5$ & 6.15 & 6.4 & 3.99 & 4.15 & 2.8 & 3 & 23.97 & 14.72 & 10.39 & 6 & 5.09 & 3.15 \\
\hline & $2 / 5$ & 7.22 & 6.61 & 4.61 & 4.1 & 3.37 & 3.05 & 34.41 & 16.19 & 13.98 & 6.08 & 7.32 & 3.32 \\
\hline & $3 / 5$ & 7.92 & 6.91 & 4.89 & 4.11 & 3.65 & 3.11 & 43.01 & 17.88 & 15.75 & 6.17 & 8.64 & 3.51 \\
\hline & $4 / 5$ & 8.84 & 7.55 & 5.28 & 4.33 & 3.89 & 3.13 & 55.56 & 21.93 & 18.64 & 6.99 & 9.77 & 3.58 \\
\hline \multirow[t]{4}{*}{ Joe } & $1 / 5$ & 6.91 & 6.84 & 4.51 & 4.49 & 3.23 & 3.11 & 30.72 & 17 & 13.43 & 7.17 & 6.75 & 3.5 \\
\hline & $2 / 5$ & 8.18 & 7.33 & 5.29 & 4.71 & 3.9 & 3.26 & 45.88 & 19.59 & 18.81 & 8.04 & 9.84 & 3.86 \\
\hline & $3 / 5$ & 9.12 & 7.74 & 5.74 & 4.96 & 4.24 & 3.37 & 58.16 & 22.54 & 22.55 & 8.99 & 11.89 & 4.13 \\
\hline & $4 / 5$ & 10.85 & 8.88 & 6.45 & 5.44 & $\begin{array}{r}.24 \\
4.6\end{array}$ & 3.6 & 84.1 & 31.69 & 28.98 & 11.25 & 14.08 & 4.64 \\
\hline
\end{tabular}

Table 15: Average Integrated relative absolute error (IRAEx100) and Integrated squared error (ISEx10000) of $A_{n, c}^{\mathrm{CFG}}$ for 10-dimensional Archimax copula $C_{\psi, A}$ samples of size $n \in\{200,500,1000\}$. The Pickands dependence function $A$ is either DSM or LG. Parameters for the DSM case are reported in Equation (11.3). The parameter in LG is set $\varrho=1.56$ so that the averaged pairwise Kendall's tau of both Pickands dependence functions is approximately equal to 0.36. There are four choices for the Archimedean generator $\psi$, Clayton, Frank, Gumbel and Joe, each with four parameter choices so that $\tau(\psi) \in\{1 / 5,2 / 5,3 / 5,4 / 5\}$, where $\tau(\psi)=\tau\left(C_{\psi}\right)$ is Kendall's tau of the bivariate Archimedean copula $C_{\psi}$. There are 1000 Monte Carlo replicates. 


\section{References}

[1] M. Abramowitz and I. A. Stegun. Handbook of mathematical functions with formulas, graphs, and mathematical tables, volume 55 of National Bureau of Standards Applied Mathematics Series. For sale by the Superintendent of Documents, U.S. Government Printing Office, Washington, D.C., 1964.

[2] T. Bacigál and R. Mesiar. 3-dimensional Archimax copulas and their fitting to real data. In COMPSTAT 2012, Limassol, Cyprus, 2012.

[3] T. Bacigál, V. Jágr, and R. Mesiar. Non-exchangeable random variables, Archimax copulas and their fitting to real data. Kybernetika, 47:519-531, 2011.

[4] L. R. Belzile and J. G. Nešlehová. Extremal attractors of liouville copulas. J. Multivariate Anal., 160(Supplement C):68 - 92, 2017. ISSN 0047-259X. doi: https://doi.org/10.1016/j.jmva.2017.05.008. URL http: //www.sciencedirect.com/science/article/pii/S0047259X17300453.

[5] N. Ben Ghorbal, C. Genest, and J. Nešlehová. On the Ghoudi, Khoudraji, and Rivest test for extreme-value dependence. Canad. J. Statist., 37(4): 534-552, 2009. ISSN 0319-5724. URL https://doi.org/10.1002/cjs. 10034 .

[6] B. Berghaus, A. Bücher, and S. Volgushev. Weak convergence of the empirical copula process with respect to weighted metrics. Bernoulli, 23(1):743772, 2017. ISSN 1350-7265. URL https://doi.org/10.3150/15-BEJ751.

[7] N. H. Bingham, C. M. Goldie, and J. L. Teugels. Regular variation, volume 27 of Encyclopedia of Mathematics and its Applications. Cambridge University Press, Cambridge, 1989. ISBN 0-521-37943-1.

[8] P. Capéraà, A.-L. Fougères, and C. Genest. A nonparametric estimation procedure for bivariate extreme value copulas. Biometrika, 84:567-577, 1997.

[9] P. Capéraà, A.-L. Fougères, and C. Genest. Bivariate distributions with given extreme value attractor. J. Multivariate Anal., 72(1):30-49, 2000.

[10] A. Charpentier and J. Segers. Tails of multivariate Archimedean copulas. J. Multivariate Anal., 100:1521-1537, 2009.

[11] A. Charpentier, A.-L. Fougères, C. Genest, and J. G. Nešlehová. Multivariate Archimax copulas. J. Multivariate Anal., 126:118-136, 2014. ISSN 0047-259X. doi: 10.1016/j.jmva.2013.12.013. URL http://dx.doi.org/ $10.1016 / j \cdot j m v a .2013 .12 .013$.

[12] S. Coles, J. Heffernan, and J. Tawn. Dependence measures for extreme value analyses. Extremes, 2:4:339-365, 1999. 
[13] J. Einmahl, A. Kiriliouk, and J. Segers. A continuous updating weighted least squares estimator of tail dependence in high dimension. Extremes, pages 1-29, 2017. doi: 10.1007/s10687-017-0303-7.

[14] P. Embrechts, C. Klüppelberg, and T. Mikosch. Modelling Extremal Events for Insurance and Finance. Springer, New York, 1997.

[15] A.-L. Fougères, C. Mercadier, and J. P. Nolan. Dense classes of multivariate extreme value distributions. J. Multivariate Anal., 116:109-129, 2013. ISSN 0047-259X. doi: 10.1016/j.jmva.2012.11.015. URL http://dx.doi.org/ 10.1016/j.jmva.2012.11.015.

[16] A.-L. Fougères, L. de Haan, and C. Mercadier. Bias correction in multivariate extremes. Ann. Statist., 43:903-934, 2015. doi: 10.1214/14-AOS1305.

[17] C. Genest and K. Ghoudi. Une famille de lois bidimensionnelles insolite. C. R. Acad. Sci. Paris Sér. I Math., 318(4):351-354, 1994.

[18] C. Genest and L.-P. Rivest. Statistical inference procedures for bivariate Archimedean copulas. J. Amer. Statist. Assoc., 88:1034-1043, 1993.

[19] C. Genest and J. Segers. Rank-based inference for bivariate extreme-value copulas. Ann. Statist., 37(5B):2990-3022, 2009. ISSN 0090-5364. doi: 10.1214/08-AOS672. URL http://dx.doi.org/10.1214/08-A0S672.

[20] C. Genest, J. Nešlehová, and J. Ziegel. Inference in multivariate Archimedean copula models. TEST, 20:223-256, 2011.

[21] K. Ghoudi, A. Khoudraji, and L.-P. Rivest. Propriétés statistiques des copules de valeurs extrêmes bidimensionnelles. Canad. J. Statist., 26(1):187197, 1998. ISSN 0319-5724. URL https://doi.org/10.2307/3315683.

[22] G. Gudendorf and J. Segers. Nonparametric estimation of an extreme-value copula in arbitrary dimensions. J. Multivariate Anal., 102:37-47, 2011.

[23] G. Gudendorf and J. Segers. Nonparametric estimation of multivariate extreme-value copulas. J. Statist. Plann. Inference, 142:3073-3085, 2012.

[24] P. Hall and N. Tajvidi. Distribution and dependence-function estimation for bivariate extreme-value distributions. Bernoulli, 6:835-844, 2000.

[25] M. Hofert. Sampling Archimedean copulas. Comput. Statist. Data Anal., 52(12):5163-5174, 2008.

[26] M. Hofert and M. Maechler. Parallel and other simulations in R made easy: An end-to-end study. J. Statist. Software, 69(4):1-44, 2016. doi: 10.18637/jss.v069.i04.

[27] X. Huang. Statistics of bivariate extreme values. Tinbergen Institute Research Series 22, 1992. Thesis (Ph.D.)-Erasmus University Rotterdam. 
[28] R. Huser, T. Opitz, and E. Thibaud. Bridging asymptotic independence and dependence in spatial extremes using Gaussian scale mixtures. Spat. Stat., 21(part A):166-186, 2017. ISSN 2211-6753. URL https://doi.org/ 10.1016/j.spasta.2017.06.004.

[29] H. Joe. Multivariate models and dependence concepts, volume 73 of Monographs on Statistics and Applied Probability. Chapman \& Hall, London, 1997. ISBN 0-412-07331-5. doi: 10.1201/b13150. URL http: //dx.doi.org/10.1201/b13150.

[30] H. Joe. Dependence Modeling With Copulas. CRC Press, Boca Raton, FL, 2015.

[31] M. G. Kendall and B. Babington Smith. On the method of paired comparisons. Biometrika, 31:324-345, 1940.

[32] I. Kojadinovic, J. Segers, and J. Yan. Large-sample tests of extreme-value dependence for multivariate copulas. Canad. J. Statist., 39(4):703-720, 2011. ISSN 0319-5724. URL https://doi.org/10.1002/cjs.10110.

[33] M. Larsson and J. Nešlehová. Extremal behavior of Archimedean copulas. Adv. in Appl. Probab., 43(1):195-216, 2011. ISSN 0001-8678. doi: 10.1239/ aap/1300198519. URL http://dx.doi.org/10.1239/aap/1300198519.

[34] A. W. Ledford and J. A. Tawn. Statistics for near independence in multivariate extreme values. Biometrika, 83(1):169-187, 1996. ISSN 00063444. doi: 10.1093/biomet/83.1.169. URL http://dx.doi.org/10.1093/ biomet/83.1.169.

[35] S. Malov. On finite-dimensional archimedean copulas. In N. Balakrishnan, I. Ibragimov, and V. Nevzorov, editors, Asymptotic methods in probability and statistics with applications, pages 19-35, Basel, 2001. Birkhäuser.

[36] A. J. McNeil and J. Nešlehová. Multivariate Archimedean copulas, $d$ monotone functions and $l_{1}$-norm symmetric distributions. Ann. Statist., 37(5B):3059-3097, 2009. ISSN 0090-5364. URL https://doi.org/10. 1214/07-A0S556.

[37] R. Mesiar and V. Jágr. $d$-dimensional dependence functions and Archimax copulas. Fuzzy Sets and Systems, 228:78-87, 2013.

[38] P. M. Morillas. A characterization of absolutely monotonic $(\Delta)$ functions of a fixed order. Publ. Inst. Math. (Beograd) (N.S.), 78(92):93-105, 2005.

[39] P. Naveau, R. Huser, P. Ribereau, and A. Hannart. Modeling jointly low, moderate, and heavy rainfall intensities without a threshold selection. Water Resour. Res., 52:1-17, 2016.

[40] R. B. Nelsen. An Introduction to Copulas. Springer Series in Statistics. Springer, New York, second edition, 2006. ISBN 978-0387-28659-4; 0-38728659-4. 
[41] J. Pickands, III. Multivariate extreme value distributions. In Proceedings of the 43rd session of the International Statistical Institute, Vol. 2 (Buenos Aires, 1981), volume 49, pages 859-878, 894-902, 1981.

[42] S. I. Resnick. Extreme values, regular variation, and point processes, volume 4 of Applied Probability. A Series of the Applied Probability Trust. $\quad$ Springer-Verlag, New York, 1987. ISBN $0-387-96481-9$. doi: 10.1007/978-0-387-75953-1. URL http://dx.doi.org/10.1007/ 978-0-387-75953-1.

[43] P. Ressel. Homogeneous distributions - and a spectral representation of classical mean values and stable tail dependence functions. J. Multivariate Anal., 117:246-256, 2013.

[44] J. Segers. Asymptotics of empirical copula processes under non-restrictive smoothness assumptions. Bernoulli, 18(3):764-782, 2012. ISSN 1350-7265. doi: 10.3150/11-BEJ387. URL http://dx.doi.org/10.3150/11-BEJ387.

[45] A. Sklar. Fonctions de répartition à $n$ dimensions et leurs marges. Publ. Inst. Statist. Univ. Paris, 8:229-231, 1959.

[46] A. W. van der Vaart and J. A. Wellner. Weak convergence and empirical processes. Springer Series in Statistics. Springer-Verlag, New York, 1996. ISBN 0-387-94640-3. doi: 10.1007/978-1-4757-2545-2. URL http://dx. doi.org/10.1007/978-1-4757-2545-2. With applications to statistics.

[47] J. L. Wadsworth, J. A. Tawn, A. C. Davison, and D. M. Elton. Modelling across extremal dependence classes. J. R. Stat. Soc. Ser. B. Stat. Methodol., 79(1):149-175, 2017. ISSN 1369-7412. URL https://doi.org/10.1111/ rssb. 12157.

[48] R. E. Williamson. Multiply monotone functions and their Laplace transforms. Duke Math. J., 23:189-207, 1956. ISSN 0012-7094.

[49] D. Zhang, M. T. Wells, and L. Peng. Nonparametric estimation of the dependence function for a multivariate extreme value distribution. J. Multivariate Anal., 99:577-588, 2008. 